\title{
Tortuosity of porous media: Image analysis and physical simulation
}

\author{
Jinlong $\mathrm{Fu}^{\mathrm{a}}$, Hywel R. Thomas ${ }^{\mathrm{a}}$, and Chenfeng $\mathrm{Li}{ }^{* a, \mathrm{~b}}$ \\ ${ }^{a}$ Zienkiewicz Centre for Computational Engineering, College of Engineering, Swansea \\ University Bay Campus, Swansea SA1 8EN, United Kingdom \\ ${ }^{\mathrm{b}}$ Energy Safety Research Institute, College of Engineering, Swansea University Bay Campus, \\ Swansea SA1 8EN, United Kingdom
}

\begin{abstract}
Tortuosity is widely used as a critical parameter to predict transport properties of porous media, such as rocks and soils. But unlike other standard microstructural properties, the concept of tortuosity is vague with multiple definitions and various evaluation methods introduced in different contexts. Hydraulic, electrical, diffusional, and thermal tortuosities are defined to describe different transport processes in porous media, while geometrical tortuosity is introduced to characterize the morphological property of porous microstructures. In particular, the rapid development of microscopy imaging techniques has made digital microstructures of porous media increasingly accessible, from which geometrical and physical tortuosities can be evaluated using various image analysis and numerical simulation methods. These tortuosities are defined differently and can differ greatly in value, but in many works of literature, they are used interchangeably. To address this situation, we systematically examine geometrical, hydraulic, electrical, diffusional, and thermal tortuosities from the viewpoints of the definition and evaluation method. For the same porous medium, visible discrepancies are found between the evaluated geometrical and physical tortuosities, depending on the specific definition and the evaluation method adopted. This observation makes it questionable to directly use the geometrical tortuosity as a substitute for physical tortuosities, a common practice in the literature. Thus, the correlations between geometrical and physical tortuosities are further analyzed, which also takes into account the influence of both image size and resolution. From the correlation analysis, phenomenological relations between geometrical and physical tortuosities are established, so that the latter can be accurately predicted by using the former that is much cheaper to evaluate from digital microstructures.
\end{abstract}

Keywords: Tortuosity; Porous media; Image analysis; Numerical simulation; Microstructural characteristic; Transport property.

\section{Contents}

1 Introduction $\mathbf{2}$

1.1 Experimental measurement ............................ 3

1.2 Theoretical/phenomenological models . . . . . . . . . . . . . . . . 3

1.3 Image analysis ............................... 5

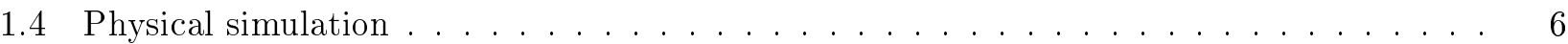

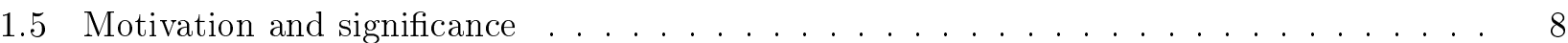

${ }^{*}$ Corresponding author: c.f.li@swansea.ac.uk 
2 Geometrical tortuosity 9

2.1 Definition of geometrical tortuosity . . . . . . . . . . . . . . . . . . . 9

2.2 Direct shortest-path search method . . . . . . . . . . . . . . . . . . . . . . . 10

2.3 Skeleton shortest-path search method . . . . . . . . . . . . . . . . . . . . . 11

2.4 Fast marching method . . . . . . . . . . . . . . . . . . . . . . . . . . 12

2.5 Pore centroid method ............................. . . . . . . . . . . . . . .

3 Physical tortuosities 13

3.1 Analogy among different transport phenomena . . . . . . . . . . . . . . . . . . . . . 14

3.2 Hydraulic tortuosity . . . . . . . . . . . . . . . . . . . . . . . . . 15

3.2.1 Definition of hydraulic tortuosity . . . . . . . . . . . . . . . . . . . 15

3.2.2 Evaluation via fluid flow simulation . . . . . . . . . . . . . . . . . . . . . 15

3.3 Electrical tortuosity . . . . . . . . . . . . . . . . . . . . . 16

3.3.1 Definition of electrical tortuosity . . . . . . . . . . . . . . . . 16

3.3.2 Evaluation via electrical conduction simulation . . . . . . . . . . . . . . . 17

3.4 Diffusional tortuosity . . . . . . . . . . . . . . . . . . . . . . 18

3.4.1 Definition of diffusional tortuosity ...................... . . . 18

3.4.2 Evaluation via molecular diffusion simulation . . . . . . . . . . . . . . . . . 19

3.4.3 Evaluation via random walk simulation .................... 20

3.5 Thermal tortuosity . . . . . . . . . . . . . . . . . . . . . . . 21

3.5.1 Definition of thermal tortuosity . . . . . . . . . . . . . . . . . . 21

3.5.2 Evaluation via heat transfer simulation . . . . . . . . . . . . . . . . . . 22

4 Comparison and analysis $\quad \mathbf{2 3}$

4.1 Comparison between geometrical tortuosity results . . . . . . . . . . . . . . . . 24

4.2 Comparison between physical tortuosity results . . . . . . . . . . . . . . . . 26

4.3 Performances of theoretical/phenomenological models . . . . . . . . . . . . . . 27

5 Relationships between geometrical and physical tortuosities 29

5.1 Prediction model for hydraulic tortuosity . . . . . . . . . . . . . . . . . . . . . 32

5.2 Prediction model for diffusional tortuosity . . . . . . . . . . . . . . . . . . . . 33

6 Issues on REV size and resolution 34

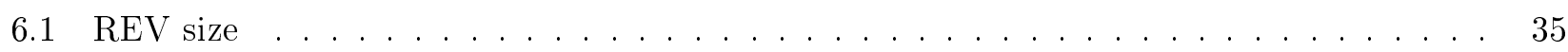

6.2 REV resolution ............................ . . . . . . . . . . . . . . . . . .

7 Discussion and concluding remarks $\mathbf{3 9}$

7.1 Discussion . . . . . . . . . . . . . . . . . . . . . . . . . 39

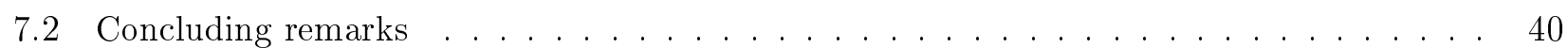

\section{Introduction}

Porous media, whether natural or artificial, comprise chaotic microstructures so that the internal flow path for fluid permeation, molecular diffusion, electrical conduction, and heat transfer are tortuous and meandering (Civan, 2011; Bear and Bachmat, 2012). In order to quantify the transport behavior of flow passing through porous media, an accurate understanding of the heterogeneous microstructure is required (Adler, 1992; Blunt et al., 2013; Bultreys et al., 2016). Such microstructural descriptors as porosity (Koponen et al., 1997), specific surface area (Petersen et al., 1996), correlation functions (Adler et al., 1990; Coker et al., 1996; Hilfer, 2002), pore size distribution (Münch and Holzer, 2008), Minkowski functionals (Vogel et al., 2010; Armstrong et al., 2019), constrictivity (Holzer et al., 2013; Keller et al., 2015) and tortuosity (Berg, 2014; Yang et al., 2019) play vital roles in characterizing the morphology of porous media. Among them, tortuosity is a special parameter, which is not only used 
to explicitly describe the sinuosity and complexity of internal percolation paths but also to quantify transport properties of porous media (Clennell, 1997; Barrande et al., 2007; Ghanbarian et al., 2013).

Tortuosity is an important concept in many scientific and engineering fields. In geoscience, tortuosity is closely related to the transport behavior of fluid permeation, molecular diffusion, electrical conduction, and heat transfer, thereby affecting such petrophysical properties as permeability, effective diffusivity, formation resistivity factor, and thermal conductivity (Adler et al., 1990; Thovert et al., 1990; Adler, 1992; Masís-Meléndez et al., 2014; Backeberg et al., 2017; Srisutthiyakorn and Mavko, 2017). In energy storage and conversion, tortuosity is a key factor for electrodes design to maximize battery capacity (Coelho et al., 1996; Kehrwald et al., 2011; Bae et al., 2013; Zacharias et al., 2013). For chemical catalysts, tortuosity has a great influence on reaction efficiency (Prachayawarakorn and Mann, 2007; Kolitcheff et al., 2017). In acoustic applications, tortuosity is closely linked to sound propagation and velocity variation in porous structures (Corapcioglu, 1996; Zielinski, 2012; Pazdniakou and Adler, 2012). For water treatment, the tortuosity of the membrane microstructure significantly influences the distillation performance (Karanikola et al., 2017). In bone tissue engineering, tortuosity affects cell migration through the scaffold, nutrient diffusion, waste removal, and bone remodeling (Botchwey et al., 2003; Gariboldi and Best, 2015; Bini et al., 2019).

Despite this broad presence in diverse fields, tortuosity is not consistently defined by geologists, engineers, chemists and biologists. Extensive efforts have been made to determine the tortuosity of porous media, but the evaluation methods and results differ between various types of tortuosity. Also, different types of tortuosity are often interchangeably used in literature (Shen and Chen, 2007; Ghanbarian et al., 2013; Tjaden et al., 2018). In the following subsections, different approaches to determining the tortuosity of porous media are briefly reviewed, including experimental measurement, theoreti$\mathrm{cal} /$ phenomenological modeling, image analysis and physical simulation.

\subsection{Experimental measurement}

To model the permeability of porous media, the concept of tortuosity was first introduced by Kozeny (1927) based on a capillary tube experiment and then further refined by Carman (1937). Tortuosity was initially only used as a tuning parameter to account for the effect of complicated pore structures on fluid flows in porous media (Barrande et al., 2007; Borujeni et al., 2013). The presence of the solid phase in porous media causes the flow path to deviate from the straight line; and to approximate the effective flow path, the length scale of the pore channel is adjusted by tortuosity. Besides fluid permeation, the tortuosity concept has been applied to other transport processes in porous media as well, including molecular diffusion, electrical conduction and heat transfer.

There are two widely-used experimental approaches to measuring the tortuosity of porous media. The first approach is based on the electrical conductivity experiment, where the test porous media sample is saturated with an electrolyte and the tortuosity is evaluated using the effective conductivity measured on the porous sample (Garrouch et al., 2001; Weerts et al., 2001; Barrande et al., 2007). The second approach measures the diffusion coefficient of a nonreactive species diffusing through the porous media sample, and the tortuosity is estimated using the measured diffusivity (Barrande et al., 2007; Weerts et al., 2001; He et al., 2013). Compared to the electrical conductivity experiment, the diffusion process in porous media is much slower, and the corresponding experiment is sensitive to the selected chemical species and temperature. Typically, the tortuosity values measured from electrical conductivity and diffusion experiments can be different (Garrouch et al., 2001). Therefore, different tortuosities are defined depending on the underlying physical process and the measurement protocol (Yang et al., 2019).

\subsection{Theoretical/phenomenological models}

A large number of theoretical or phenomenological models have been proposed to estimate the tortuosity of porous media, and most of them simply express tortuosity as functions of porosity (Mota et al., 2001; Lanfrey et al., 2010; Duda et al., 2011; Ahmadi et al., 2011). The Bruggeman correlation (Bruggeman, 
1935; Dias et al., 2006) is one of the most commonly used porosity-tortuosity relationships, which is expressed as an exponential equation, given by

$$
\tau^{2}=\phi^{1-\alpha}
$$

where $\tau$ denotes the tortuosity, $\phi$ is the porosity and $\alpha$ is an empirical constant called Bruggeman exponent. Considering the relationship between porosity $\phi$ and formation resistivity factor $F$, i.e. $\tau^{2}=\phi F$ (Wyllie et al., 1950; Coleman and Vassilicos, 2008), the Bruggeman relation is formally consistent with the well-known Archie's law (Archie et al., 1942): $F=\phi^{-\alpha}$. Another famous model was presented by Comiti and Renaud (1989), which treats tortuosity as a logarithmic function of porosity:

$$
\tau^{2}=1-p \ln \phi
$$

where $p$ is an empirical constant related to the grain shape. The porosity-tortuosity model put forward by Iversen and Jørgensen (1993) is also popular, where the linear relationship between porosity and tortuosity was observed as follows:

$$
\tau^{2}=1+q(1-\phi)
$$

where $q$ is an empirical constant depending on the type of porous media. Du Plessis and Masliyah (1991) derived an analytical model from an idealized granular pore microstructure without involving any undetermined parameter, which is mathematically expressed as

$$
\tau=\frac{\phi}{1-(1-\phi)^{2 / 3}}
$$

Besides, another representative theoretical model was derived from a fixed bed of randomly packed identical particles by Lanfrey et al. (2010), given by

$$
\tau=1.23 \frac{(1-\phi)^{4 / 3}}{\xi^{2} \phi}
$$

where $\xi$ is the shape factor (sphericity) of particle, $\xi=1$ for sphere and $\xi<1$ for non-spherical particles.

The above five porosity-tortuosity relations are representatives of various models for tortuosity estimations. Despite the simple formulations, phenomenological models usually contain empirical parameters or uncertain coefficients such as $\alpha, p, q$ and $\xi$ in above equations, and accurate determinations of them are difficult. Even for a small class of porous media, these adjustable parameters can vary significantly causing great errors for the tortuosity estimation (Ghanbarian et al., 2013; Tjaden et al., 2018). To improve the prediction accuracy, tortuosity models with more sophisticated formulation and more parameters have been proposed, but typically they are only suitable for a narrow range of microstructures (Pisani, 2011; Matyka et al., 2008; Lanfrey et al., 2010; Chung et al., 2013). Ghanbarian et al. (2013) presented a critical review of tortuosity models including geometrical, hydraulic, electrical and diffusional tortuosities, and it was found that various porosity-tortuosity models differ greatly and may not be used interchangeably. Moreover, although many actual porous media are anisotropic in nature, most theoretical and phenomenological models assume the medium to be isotropic.

Microstructural features such as the shape, size, orientation and spatial distribution of grains and pores all affect the tortuosity of porous media (Vervoort and Cattle, 2003), but most phenomenological models rely mainly on the porosity (Shen and Chen, 2007; Ghanbarian et al., 2013). A porous medium contains a large number of geometrically irregular pore bodies, some are connected, and others are isolated or dead-end. The length scales of randomly distributed pores can range over several orders of magnitude. Besides, the solid-pore interfaces are typically rough with a significant resistance effect on transport flow. These complications make it extremely challenging, if not impossible, to develop an explicit mathematical model that can accurately predict the tortuosity for general porous media. Indeed, the tortuosity values calculated from various phenomenological models often differ greatly from experimental measurements or numerical simulation results and are even incorrect in some cases (Shen and Chen, 2007; Matyka et al., 2008; Lanfrey et al., 2010; Tjaden et al., 2018). 


\subsection{Image analysis}

As illustrated in Figure 1, modern microscopy imaging techniques are capable of providing high-fidelity visualization and characterization of $2 \mathrm{D} / 3 \mathrm{D}$ porous microstructures at scales of nanometers to millimeters (Lai et al., 2018), and they include X-ray micro-computed tomography (Micro-CT) (Spanne et al., 1994; Schlüter et al., 2014), focused ion beam and scanning electron microscopy (FIB-SEM) (Munroe, 2009), scanning electron microscopy (SEM)(Stutzman, 2004), optical microscopy (Georgali and Tsakiridis, 2005), transmission electron microscopy (TEM) (Ringer and Hono, 2000), atomic force microscopy (AFM) (Javadpour et al., 2009), magnetic resonance imaging (MRI) (Anovitz and Cole, 2015) and small-angle X-ray scattering (SAXC) (Zhao et al., 2014). Although the imaging mechanisms of these techniques are different, the acquired digital images are comparable at the same resolution levels. Raw microscopic images are typically processed and stored as pixels or voxels in gray scale (as shown in Figure 20a), and through image segmentation they can be converted into binary forms, where the pore space is separated from the solid matrix (as shown Figure 20c). The segmented digital microstructure with sufficient size and adequate resolution can be directly used as a representative elementary volume (REV) for further analyses of geometrical characterization and physical simulation.

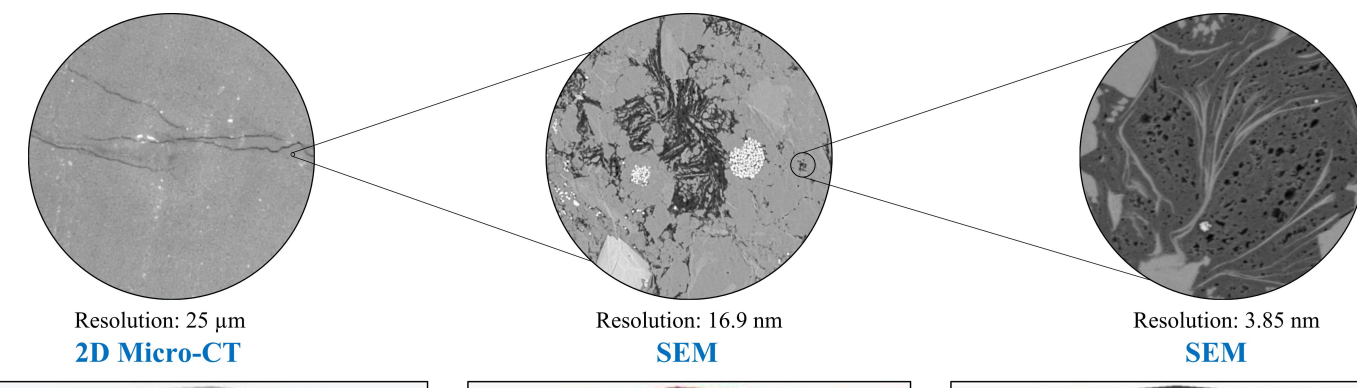

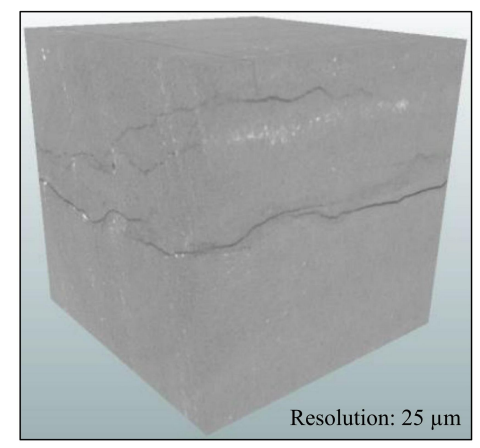

Micro-CT

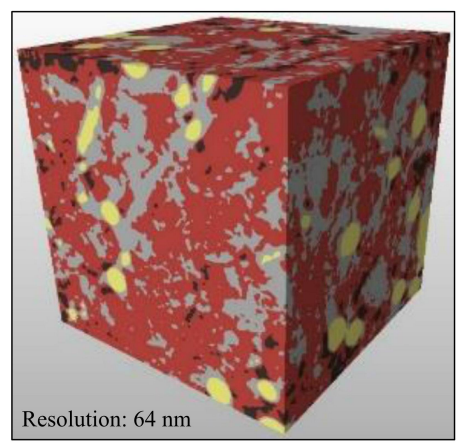

Nano-CT

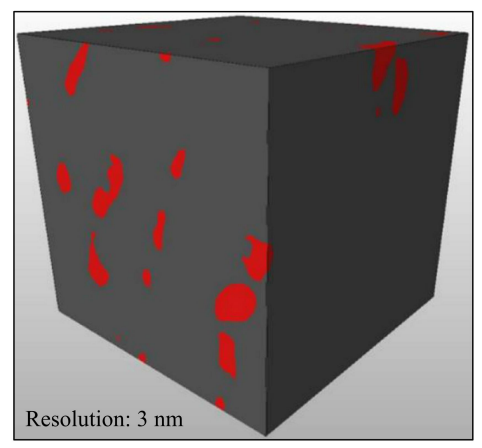

FIB-SEM

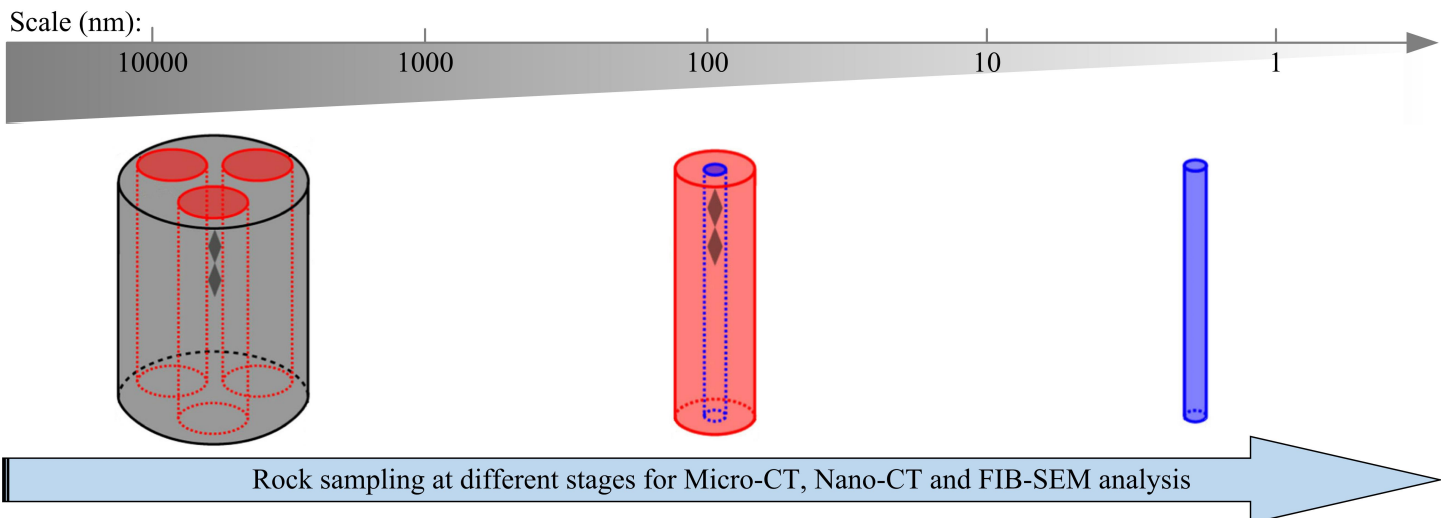

Figure 1: Microstructure characterization at different length scales via advanced microscopy imaging techniques (the raw picture materials are collected from Ning et al. (2016) and Devarapalli et al. (2017)).

With the increasing availability of digital microstructures, the effects of complex microstructures on the macroscopic transport properties have attracted significant attention in recent years (Blunt et al., 2013; Wildenschild and Sheppard, 2013; Bultreys et al., 2016). Owing to the high efficiency, it is 
desirable to directly evaluate the tortuosity through image analysis. Thus, to describe the degree of flow paths deviating from straight lines in porous media, the geometrical tortuosity is defined as the ratio of the effective flow-path length to the straight-line distance in the macroscopic flow direction (Ghanbarian et al., 2013). The geometrical tortuosity is usually computed by considering the shortest pore channels as the actual flow paths through porous media (Sun et al., 2011; Cecen et al., 2012). Various algorithms, such as Dijkstra's algorithm and fast marching algorithm, have been used to search for the shortest pore channels that can be equivalent to the effective flow paths. The popular image-based methods to calculate the geometrical tortuosity are listed in Table 1, including the direct shortest path searching method (DSPSM), the skeleton shortest path searching method (SSPSM), the fast marching method (FMM) and the pore centroid method (PCM), etc.

Table 1: Popular image analysis methods to calculate geometrical tortuosity.

\begin{tabular}{|c|c|c|c|}
\hline Evaluation method & Porous media & Dimension & Reference \\
\hline $\begin{array}{l}\text { DSPSM } \\
\text { (Dijkstra's algorithm) }\end{array}$ & $\begin{array}{l}\text { Porous rocks, } \\
\text { nanocomposites, and } \\
\text { fuel cell electrodes. }\end{array}$ & $3 \mathrm{D}$ & $\begin{array}{l}\text { Thovert et al. (1993); } \\
\text { Gommes et al. (2009); } \\
\text { Cecen et al. (2012); } \\
\text { Wargo et al. (2013); } \\
\text { Litster et al. (2013); } \\
\text { Stenzel et al. (2016). }\end{array}$ \\
\hline $\begin{array}{l}\text { DSPSM } \\
\text { (Wavefront propagation } \\
\text { algorithm) }\end{array}$ & $\begin{array}{l}\text { Structures of sodium } \\
\text { chloride compacts. }\end{array}$ & $2 \mathrm{D}$ & San Wu et al. (2006). \\
\hline $\begin{array}{l}\text { DSPSM } \\
\text { (A-star algorithm) }\end{array}$ & $\begin{array}{l}\text { Structures of } \\
\text { sintered compacts. }\end{array}$ & $2 \mathrm{D}$ & Montes et al. (2007). \\
\hline $\begin{array}{l}\text { SSPSM } \\
\text { (Dijkstra's algorithm) }\end{array}$ & $\begin{array}{l}\text { Porous rocks, } \\
\text { fuel cell electrodes, } \\
\text { alumina, and paper } \\
\text { microstructures. }\end{array}$ & $3 \mathrm{D}$ & $\begin{array}{l}\text { Lindquist et al. (1996); } \\
\text { Axelsson and Svensson (2010); } \\
\text { Sun et al. (2011); } \\
\text { Shanti et al. (2014); } \\
\text { Al-Raoush and Madhoun (2017). }\end{array}$ \\
\hline FMM & $\begin{array}{l}\text { Porous rocks, } \\
\text { fuel cell electrodes, } \\
\text { infiltrated aluminium, } \\
\text { cellular materials, and } \\
\text { energy storage materials. }\end{array}$ & $3 \mathrm{D}$ & $\begin{array}{l}\text { Brun et al. (2008); } \\
\text { Jørgensen et al. (2011); } \\
\text { Pardo-Alonso et al. (2014); } \\
\text { Chen-Wiegart et al. (2014); } \\
\text { Taiwo et al. (2016). }\end{array}$ \\
\hline PCM & $\begin{array}{l}\text { Porous rocks, } \\
\text { soil and, fuel } \\
\text { cell electrodes. }\end{array}$ & $3 \mathrm{D}$ & $\begin{array}{l}\text { Gostovic et al. (2007); } \\
\text { Desrues et al. (2010); } \\
\text { Shearing et al. (2012); } \\
\text { Kaczmarek et al. (2017). }\end{array}$ \\
\hline Path tracking method & Artificial models. & $3 \mathrm{D}$ & Sobieski et al. (2018). \\
\hline
\end{tabular}

It should be noted that geometrical tortuosity is a microstructural characteristic, independent from the specific transport process in porous media (Clennell, 1997). Moreover, although constrictions and bottlenecks of pore channels can greatly affect the transport behaviors (Holzer et al., 2013; Berg, 2014), geometrical tortuosity only considers the longitudinal distance of possible flow paths and neglects the flow variation in the cross-section of pore channels. Also, the flow paths identified by image analysis can differ greatly from the real flow paths in porous media (Ghanbarian et al., 2013). These limitations may explain why geometrical tortuosity often fails to accurately model/predict such transport properties of porous media as permeability, effective diffusivity and conductivity.

\subsection{Physical simulation}

High-quality digital microstructures also make it possible to mimic experimental measurements through high-fidelity numerical simulations, so that tortuosities can be evaluated from physical simulations of fluid permeation, electrical conduction, molecular diffusion and heat transfer in porous media (Lemaitre 
Table 2: Numerical simulation methods for the prediction of physical tortuosities.

\begin{tabular}{|c|c|c|c|c|}
\hline Tortuosity type & Numerical method & Porous media & Dimension & Reference \\
\hline Hydraulic & $\begin{array}{l}\text { Lattice gas automata } \\
\text { (The precursor to LBM) }\end{array}$ & Artificial models. & $3 \mathrm{D}$ & Zhang and Knackstedt (1995). \\
\hline Hydraulic & LBM & $\begin{array}{l}\text { Porous rocks and } \\
\text { artificial models. }\end{array}$ & $2 \mathrm{D}$ and $3 \mathrm{D}$ & $\begin{array}{l}\text { Koponen et al. (1996); } \\
\text { Matyka et al. (2008); } \\
\text { Duda et al. (2011); } \\
\text { Matyka and Koza (2012); } \\
\text { Degruyter et al. (2010). }\end{array}$ \\
\hline Hydraulic & FEM & Artificial models. & $2 \mathrm{D}$ & Saomoto and Katagiri (2015). \\
\hline Hydraulic & FVM & Artificial models. & $2 \mathrm{D}$ & Luo et al. (2014). \\
\hline Electrical & $\begin{array}{l}\text { Lattice gas automata } \\
\text { (The precursor to LBM) }\end{array}$ & Artificial models. & $3 \mathrm{D}$ & Zhang and Knackstedt (1995). \\
\hline Electrical & FEM & Artificial models. & $2 \mathrm{D}$ & Saomoto and Katagiri (2015). \\
\hline Thermal & FVM & $\begin{array}{l}\text { Fuel cell } \\
\text { electrodes. }\end{array}$ & $3 \mathrm{D}$ & $\begin{array}{l}\text { Cooper et al. (2013); } \\
\text { Tjaden et al. (2016); } \\
\text { Brown et al. (2016). }\end{array}$ \\
\hline Thermal & FEM & $\begin{array}{l}\text { Fuel cell } \\
\text { electrodes. }\end{array}$ & $3 \mathrm{D}$ & Sun et al. (2016). \\
\hline Diffusional & FVM & $\begin{array}{l}\text { Porous rocks and } \\
\text { battery cathode. }\end{array}$ & $3 \mathrm{D}$ & $\begin{array}{l}\text { Hutzenlaub et al. (2013); } \\
\text { Peng et al. (2014). }\end{array}$ \\
\hline Diffusional & FEM & $\begin{array}{l}\text { Fuel cell } \\
\text { electrodes. }\end{array}$ & $3 \mathrm{D}$ & $\begin{array}{l}\text { Wilson et al. (2006); } \\
\text { Chen-Wiegart et al. (2014). }\end{array}$ \\
\hline Diffusional & FDM & $\begin{array}{l}\text { Porous rocks } \\
\text { and fuel cell } \\
\text { electrodes. }\end{array}$ & $3 \mathrm{D}$ & $\begin{array}{l}\text { Pisani (2011); } \\
\text { Cooper et al. (2016); } \\
\text { Finegan et al. (2016); } \\
\text { Backeberg et al. (2017). }\end{array}$ \\
\hline Diffusional & LBM & $\begin{array}{l}\text { Soils, and } \\
\text { fuel cell electrodes. }\end{array}$ & $3 \mathrm{D}$ & $\begin{array}{l}\text { Weerts et al. (2001); } \\
\text { Iwai et al. (2010); } \\
\text { Ostadi et al. (2010). }\end{array}$ \\
\hline Diffusional & Gauss-Seidel method & Porous alumina. & $3 \mathrm{D}$ & Shanti et al. (2014). \\
\hline Diffusional & RWM & $\begin{array}{l}\text { Porous rocks, } \\
\text { fuel cell electrodes, } \\
\text { concretes and } \\
\text { bead packs. }\end{array}$ & $2 \mathrm{D}$ and $3 \mathrm{D}$ & $\begin{array}{l}\text { Nakashima and Watanabe (2002); } \\
\text { Nakashima and Kamiya (2007); } \\
\text { Iwai et al. (2010); } \\
\text { Kishimoto et al. (2013); } \\
\text { and Tranter et al. (2019). }\end{array}$ \\
\hline
\end{tabular}

and Adler, 1990; Adler et al., 1990; Thovert et al., 1990; Adler, 1992; Ginzbourg and Adler, 1994; Duda et al., 2011; Cooper et al., 2013; Tjaden et al., 2018). These flux-based tortuosities (namely the hydraulic, electrical, diffusional and thermal tortuosity) are collectively referred to as physical tortuosities in this study. Table 2 summarizes the popular numerical methods for pore-scale simulations of various transport processes in porous media to evaluate physical tortuosities, including the lattice Boltzmann method (LBM), the finite element method (FEM), the finite volume method (FVM), the finite difference method (FDM), the random walk method (RWM) and others.

In contrast to geometrical tortuosity that exclusively depends on the porous microstructure, physical tortuosities are related to both the porous microstructure and the transport flow inside it. For a specific transport process in a porous medium, the associated physical tortuosity describes the resistance effect of the porous microstructure on transport flow. Although transport processes in porous media can be fundamentally different, physical tortuosities are often interchangeably used in the literature (Clennell, 1997). For example, the electrical tortuosity was considered to be identical to the hydraulic tortuosity (Carman, 1956; Mualem and Friedman, 1991; David, 1993; Weerts et al., 1999), and many researchers 
took the former as an approximation of the latter to model permeability of porous media (de Lima, 1995; De Lima and Niwas, 2000; Barrande et al., 2007; Saomoto and Katagiri, 2015). The diffusional tortuosity obtained from molecular diffusion simulation or random walk simulation was also used to substitute the hydraulic tortuosity in hydraulic conductivity studies of porous rocks (Nakashima and Kamiya, 2007; Masís-Meléndez et al., 2014; Peng et al., 2014; Backeberg et al., 2017). Numerical simulation of thermal conduction was adopted to evaluate the diffusional tortuosity of porous media (Cooper et al., 2013; Tjaden et al., 2016; Brown et al., 2016; Sun et al., 2016). Besides, the geometrical tortuosity is commonly used as an alternative to physical tortuosities to model transport properties of porous media (Montes et al., 2007; Sun et al., 2011; Cecen et al., 2012; Holzer et al., 2013; Berg, 2014; Kaczmarek et al., 2017; Li et al., 2017), such as permeability, effective diffusivity, formation resistivity factor and thermal conductivity.

Many open-source or commercial software packages have been developed for microstructural analysis, as summarized in Table 3. These software tools can all provide morphological characteristics or physical properties of porous media, but they adopt different methods (as listed in Table 1 and Table 2) to calculate tortuosity, which often leads to inconsistent results.

Table 3: Open-source and commercial software packages for tortuosity evaluation.

\begin{tabular}{|c|c|c|c|c|}
\hline Name & Tortuosity type & Calculation method & Nature & Reference \\
\hline iMorph & Geometrical & FMM & Open-source & Brun et al. (2008) \\
\hline Tort3D & Geometrical & DSPSM & Open-source & Cecen et al. (2012) \\
\hline TORT3D & Geometrical & SSPSM & Open-source & Al-Raoush and Madhoun (2017) \\
\hline Palabos & $\begin{array}{l}\text { Hydraulic } \\
\text { Diffusional }\end{array}$ & $\begin{array}{l}\text { LBM simulation of laminar flow } \\
\text { LBM simulation of molecular diffusion }\end{array}$ & Open-source & Palabos (2019) \\
\hline OpenLB & $\begin{array}{l}\text { Hydraulic } \\
\text { Diffusional }\end{array}$ & $\begin{array}{l}\text { LBM simulation of laminar flow } \\
\text { LBM simulation of molecular diffusion }\end{array}$ & Open-source & OpenLB (2019) \\
\hline TauFactor & Diffusional & FDM & Open-source & Cooper et al. (2016) \\
\hline PuMA & Diffusional & FDM or RWM & Open-source & Ferguson et al. (2018) \\
\hline pytrax & Diffusional & RWM & Open-source & Tranter et al. (2019) \\
\hline Avizo & $\begin{array}{l}\text { Geometrical } \\
\text { Hydraulic } \\
\text { Diffusional } \\
\text { Electrical } \\
\text { Thermal }\end{array}$ & $\begin{array}{l}\text { PCM } \\
\text { FVM simulation of laminar flow } \\
\text { FVM simulation of molecular diffusion } \\
\text { FVM simulation of electrical conduction } \\
\text { FVM simulation of heat transfer }\end{array}$ & Commercial & Avizo (2017) \\
\hline Simpleware & $\begin{array}{l}\text { Geometrical } \\
\text { Hydraulic } \\
\text { Diffusional } \\
\text { Electrical } \\
\text { Thermal }\end{array}$ & $\begin{array}{l}\text { SSPSM } \\
\text { FEM simulation of laminar flow } \\
\text { FEM simulation of molecular diffusion } \\
\text { FEM simulation of electrical conduction } \\
\text { FEM simulation of heat transfer }\end{array}$ & Commercial & Simpleware (2019) \\
\hline GeoDict & $\begin{array}{l}\text { Geometrical } \\
\text { Hydraulic } \\
\text { Diffusional } \\
\text { Electrical } \\
\text { Thermal }\end{array}$ & $\begin{array}{l}\text { DSPSM or SSPSM } \\
\text { FVM simulation of laminar flow } \\
\text { FVM simulation of molecular diffusion } \\
\text { FVM simulation of electrical conduction } \\
\text { FVM simulation of heat transfer }\end{array}$ & Commercial & GeoDict (2020) \\
\hline
\end{tabular}

\subsection{Motivation and significance}

A summary of the origin, evolution and variation of the tortuosity concept was given by Clennell (1997), where the relationships between different types of tortuosity were analyzed. The tortuosity concept varies depending on the specific application, but in general, it can be understood from two interrelated perspectives: (1) a geometrical measure to characterize the sinuosity of pore channels (flow paths) 
inside the porous medium; (2) a physical characteristic to quantify the retarding effect (resistance) of the heterogeneous microstructure on a specific transport process inside the porous medium. The nature of tortuosity has not been fully understood, so that it remains confusing whether tortuosity is an intrinsic microstructural property of the porous medium, a physical characteristic depending on the type of transport process, or just a 'fudge factor' to tune predictions to match with experimental results (Ghanbarian et al., 2013).

A diverse range of approaches have been developed and applied to evaluate tortuosities of porous media, including experimental measurement, theoretical/phenomenological models, image analysis, and physical simulation, but inconsistent results are often obtained from different evaluation methods (Garrouch et al., 2001; Shen and Chen, 2007; Ghanbarian et al., 2013; Pardo-Alonso et al., 2014; Tjaden et al., 2018). There is no rigorous and unique definition for tortuosity as its physical essence has not been completely understood. Other factors, including experimental facility and condition, modeling assumption and data, numerical simulation method and boundary condition, and image preparation and analysis methods, also have a significant influence on the tortuosity results. Further research is required to tackle the nature of tortuosity and the relationship between different evaluation approaches, which are critical for understanding the transport behaviors inside porous media.

In this study, we focus on the modern approaches to evaluating the tortuosity of porous media, i.e. image analysis and physical simulation. It is noted that the tortuosity $\tau$ is a scalar-valued factor greater than 1. Neither the tortuosity factor $\tau^{2}$ (i.e. the square of tortuosity) (Epstein, 1989) nor the tortuosity coefficient $\tau^{-1}$ (i.e. the inverse of the tortuosity) (Hillel, 2003) is considered in this work. Geometrical and physical tortuosities are systematically reviewed in $\S 2$ and $\S 3$, respectively, to clarify the definition and evaluation method of each type of tortuosity. A comparison study is conducted for the geometrical tortuosity evaluations in $\S 4.1$, where the geometrical tortuosity values obtained from different image analysis methods are found to be inconsistent. Although the geometrical tortuosity has a relatively clear definition, several uncertainties exist in its evaluation methods via image analysis. Another comparison study is presented in $\S 4.2$ for numerical simulations of different transport flows in porous media, to examine the relationship between physical tortuosities. The underlying physics is explored to explain the differences and similarities. Large discrepancies are observed between geometrical and physical tortuosities, which questions strongly the common practice of using the former as the substitute for the latter. To address this problem, empirical correlations between geometrical and physical tortuosities are established in $\S 5$ for a wide range of porous media samples. Finally, the influences from REV size and image resolution on tortuosity evaluation are discussed in $\S 6$, and the correlation length is used to determine the optimal image parameters.

\section{Geometrical tortuosity}

\subsection{Definition of geometrical tortuosity}

To describe the degree of streamlines in porous media deviating from the straight line, geometrical tortuosity is defined as the ratio of the effective length of flow paths to the straight-line distance $L$ in the macroscopic flow direction (Clennell, 1997; Ghanbarian et al., 2013). As illustrated in Figure 2, the transport flow inside porous media is shaped by the pore network, so the shortest pore channel $L_{\mathrm{g}}$ is usually adopted as the effective flow path $L_{\mathrm{h}}$ to compute the geometrical tortuosity $\tau_{\mathrm{g}}$ (Adler, 1992), given by

$$
\tau_{\mathrm{g}}=\frac{L_{\mathrm{g}}}{L}
$$

Compared with the zigzag line of the shortest pore channel, the effective flow path is much smoother.

Geometrical tortuosity can be considered as a microstructural characteristic as it is purely determined by the geometrical and morphological features of porous media (Clennell, 1997). Various image analysis methods have been developed to evaluate geometrical tortuosity from the digital microstructures of porous media, including the direct shortest-path search method (DSPSM), the skeleton shortest-path search method (SSPSM), the fast marching method (FMM) and the pore centroid method (PCM) etc. 


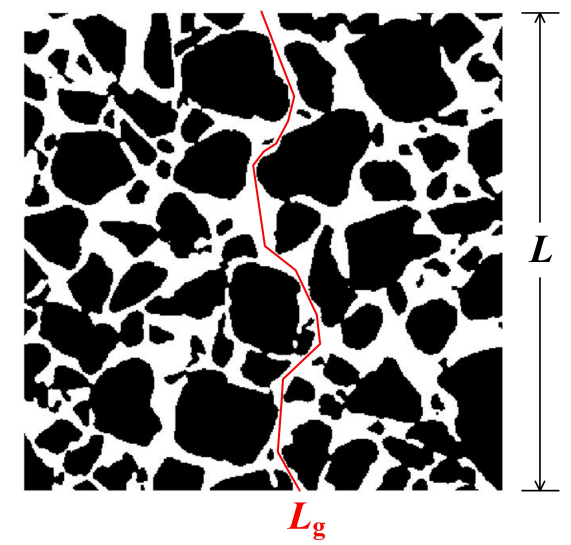

(a)

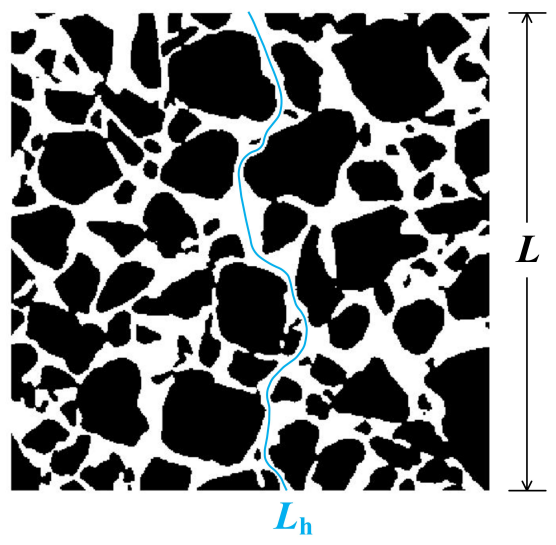

(b)

Figure 2: (a) The shortest pore-channel (geodesic) length $L_{\mathrm{g}}$, (b) the effective flow-path length $L_{\mathrm{h}}$ and the straight-line (Euclidean) distance $L$ in a $2 \mathrm{D}$ digital microstructure.

These image-based methods directly operate on the pixel/voxel data, which are usually easy to implement and computationally efficient. It is however worth to note that the geometrical tortuosity does not account for the variation of pore radius along the pore channels, although the constriction and bottleneck of porous microstructures do play a vital role in transport properties (Tjaden et al., 2018).

\subsection{Direct shortest-path search method}

The DSPSM searches for the shortest pore channel directly on the pixel/voxel data by using the shortestpath search algorithms (Cherkassky et al., 1996; Stenzel et al., 2016), such as Dijkstra's algorithm (Sun et al., 2011; Cecen et al., 2012), the wavefront propagation algorithm (Wu et al., 2006) and the Astar algorithm (Montes et al., 2007). In this subsection, a representative DSPSM based on Dijkstra's algorithm is briefly recapped.

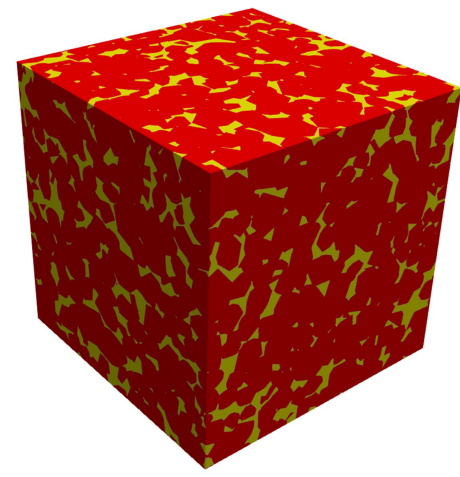

(a)

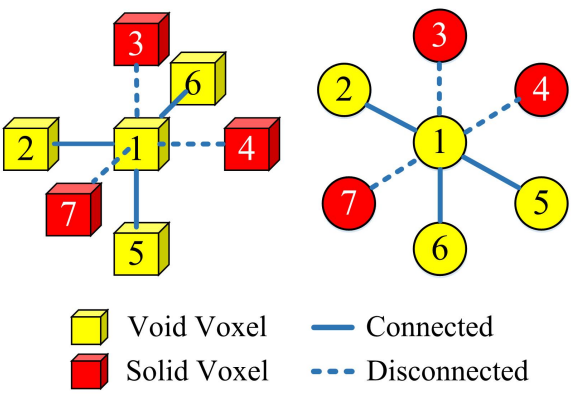

(b)

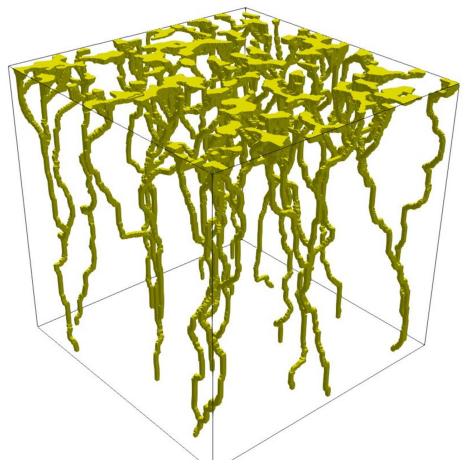

(c)

Figure 3: (a) A 3D digital microstructure (pore space is shown in yellow, and solid matrix is shown in red); (b) Conversion of 3D dataset to 2D graph for path search based on the 6-neighbor-connectivity criterion; (c) The shortest pore channels between top and bottom surfaces determined by using Dijkstra's algorithm.

The shortest pore channel is defined as the shortest connected route starting from a surface voxel on one side of the digital microstructure and ending at another surface voxel on the opposite side, and it must always remain in the pore space (Cecen et al., 2012). Changing the starting or ending voxel will lead to a different shortest path, so the geometrical tortuosity $\tau_{\mathrm{g}}$ is usually calculated as the average 
ratio of the shortest pore channel length $L_{\min }$ to the corresponding straight-line distance $L$ :

$$
\tau_{\mathrm{g}}=\left\langle\frac{L_{\min }}{L}\right\rangle
$$

The algorithm first converts the 3D microstructure dataset into a 2D adjacency matrix, so that the digital microstructure in Figure 3a is modeled by the graph in Figure 3b. Each node in the graph represents a voxel in the microstructure, and the connections between nodes represent the voxel connectivities, where the 6-neighbor-connectivity criterion is usually adopted. The Euclidean distance between two adjacent void voxels in the digital microstructure is used as the weight of linkage between the corresponding nodes in the graph, and all weights are equal to 1 for the 6-neighbor-connectivity case. Dijkstra's algorithm is then used to search for the shortest path from a designate starting node to an ending node that represents a pore voxel on the opposite surface. The search is performed for each pore voxel on the start surface to identify all pore channels of interest, as shown in Figure 3c. Finally, the geometrical tortuosity $\tau_{\mathrm{g}}$ of the porous medium in Figure 3a can be calculated according to Eq. (7).

\subsection{Skeleton shortest-path search method}

The shortest pore channels identified by the DSPSM can deviate greatly from the medial axis of pore network, especially for porous media with large porosities or high-resolution microstructures. As shown in Figure 4, the shortest path identified by Dijkstra's algorithm in the wide channel shown in Figure 4b deviates greatly from the central axis, and such deviation is much smaller for the narrow channel shown in Figure 4a. As a result, the DSPSM may underestimate the value of geometrical tortuosity. Besides, to manage computational cost, the simple 6-neighbor-connectivity criterion as shown in Figure 3b is usually adopted by the DSPSM, leading to bumpy results as shown in Figure 3c, which could introduce extra errors into the geometrical tortuosity evaluation.

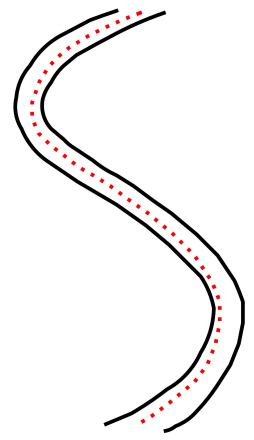

(a)

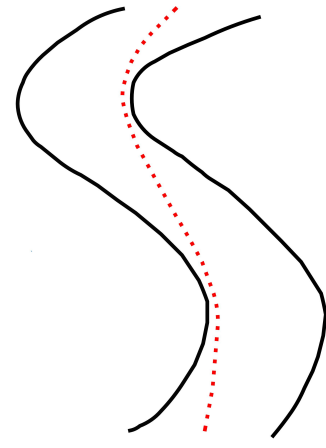

(b)

Figure 4: The shortest paths (red dashed lines) identified by Dijkstra's algorithm in a narrow pore channel (left) and a wide channel (right).

One way to reduce the above errors is to perform the path search on the pore skeleton instead of the pore space (Lindquist et al., 1996; Sun et al., 2011; Shanti et al., 2014; Al-Raoush and Madhoun, 2017). Formed by the medial axis of a pore space, the skeleton preserves to a large extent of the original topological and geometrical properties. The homotopic thinning algorithm (Thovert et al., 1993; Lee et al., 1994) is often used to extract the 3D medial axis skeleton from the digital microstructure, and then Dijkstra's algorithm is applied on the skeleton to identify the shortest pore channels, as illustrated in Figure 5. The geometrical tortuosity is then calculated according to Eq. (7). The medial axis skeleton has a much lower dimensionality compared to the original pore space, which greatly simplifies the path search task. Therefore, Dijkstra's algorithm can adopt the 18- or 26-neighbor-connectivity criterion for searching path. Generally, the tortuosity obtained from the pore skeleton is greater than that from the pore space (Stenzel et al., 2016), especially for the porous media with high porosities. More details about skeleton extraction can be found in relevant literature (Lee et al., 1994; Pudney, 1998). 


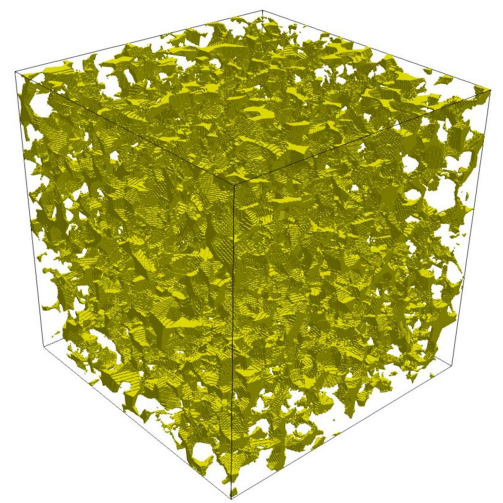

(a)

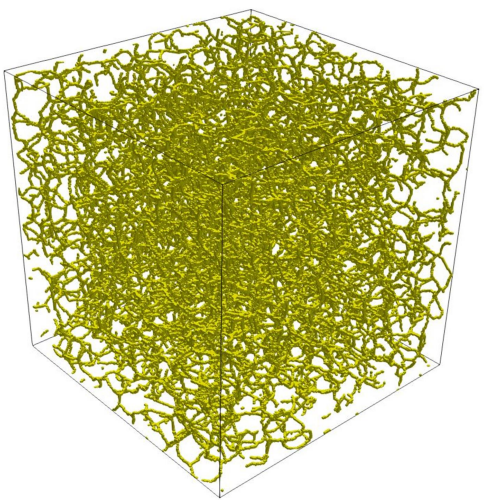

(b)

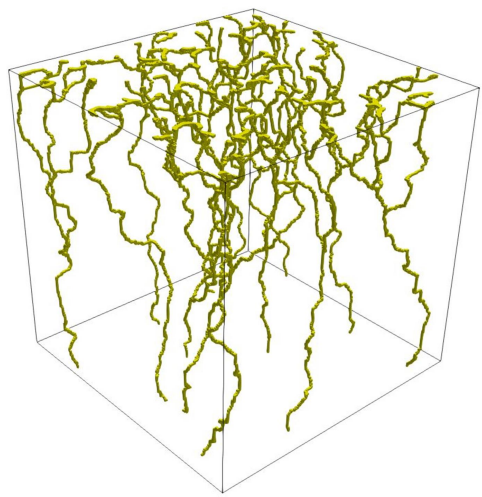

(c)

Figure 5: (a) The pore space of the 3D porous medium in Figure 3a; (b) The medial axis skeleton extracted from the pore space; (c) The shortest pore channels identified between top and bottom surfaces.

\subsection{Fast marching method}

The FMM is another frequently-used algorithm to find the shortest pore channel inside porous media (Sethian, 1996; Jørgensen et al., 2011; Hassouna and Farag, 2007; Pardo-Alonso et al., 2014; Jørgensen et al., 2015; Taiwo et al., 2016). It is an efficient computational algorithm for tracking and modeling the propagation of a wavefront moving in its normal direction with a known speed. At a given position $\mathbf{x}$, the motion of the wavefront is described by the Eikonal equation (Sethian, 1996; Bærentzen, 2001; Hassouna and Farag, 2007) as follows:

$$
\|\nabla t(\mathbf{x})\| F(\mathbf{x})=1, F(\mathbf{x})>0
$$

where $\|\nabla t(\mathbf{x})\|$ is the magnitude of the gradient vector of arrival function $t(\mathbf{x})$, and $F(\mathbf{x})$ is the propagation speed of the wavefront at the location $\mathbf{x}$. By efficiently approximating the solution to Eq. (8), the FMM calculates the time $t(\mathbf{x})$ required by the wavefront originating from the source to arrive at the other points in the computational domain. As $F(\mathbf{x})>0 \forall \mathbf{x}$, the wavefront only expands outward, and hence the points further away from the source have greater $t(\mathbf{x})$ values. If $F(\mathbf{x})=1$ is set throughout the investigation domain, the arrival time map $t(\mathbf{x})$ is equivalent to the distance map $d(\mathbf{x})$, which provides the distances to all locations from the source, as shown in Figure 6c.

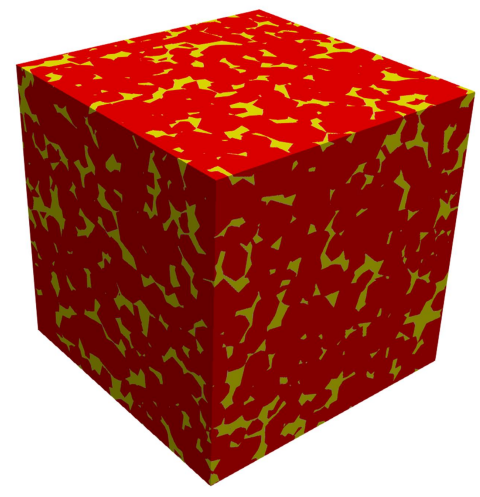

(a)

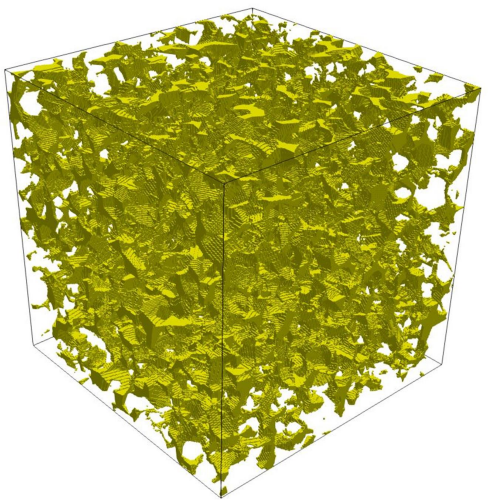

(b)



(c)

Figure 6: (a) The 3D microstructure; (b) The pore space of the 3D porous medium, which is the investigation domain of FMM; (c) The distance map calculated from FMM by setting the pore voxel on the top center as the source of wavefront expansion.

The FMM can be directly applied to a regular grid such as a 3D digital image, to evaluate the 
geometrical tortuosity $\tau_{\mathrm{g}}$ of a porous medium. The pore voxels on the starting surface are selected as the sources of wavefront expansion, and the opposite surface is set as the destination plane. The lengths of shortest pore channels $L_{\text {min }}$ connecting the source voxels and destination voxels can be obtained from the distance map $d(\mathbf{x})$, by setting $F(\mathbf{x})=1$ for the pore space and $F(\mathbf{x})=10^{-6}$ (a small value) for the solid matrix, where the wavefront moves much faster in the pore space than in the solid matrix. Similarly, the straight-line distances $L$ between the starting and ending voxels can also be obtained from the distance map $d(\mathbf{x})$, by setting $F(\mathbf{x})=1$ for both the pore space and the solid matrix, where the porous media sample is considered as a homogeneous domain allowing the wavefront to propagate freely. Once the distance data $L_{\min }$ and $L$ are determined by the FMM, the geometrical tortuosity $\tau_{\mathrm{g}}$ can be calculated according to Eq. (7).

\subsection{Pore centroid method}

The PCM estimates geometrical tortuosity $\tau_{\mathrm{g}}$ by calculating the average change of pore centroid locations between adjacent 2D slices in the 3D microstructure (Gostovic et al., 2007; Smith et al., 2009; Cooper et al., 2013; Tjaden et al., 2018). As shown in Figure 7, the effective pore channel is determined by calculating the coordinate $\left(x_{i}, y_{i}, z_{i}\right)$ of pore centroid on each layer and then connecting these pore centroids into a path. The length $L_{\mathrm{g}}$ of the effective pore path can be calculated as:

$$
L_{\mathrm{g}}=\sum_{i=1}^{N-1} L_{i}=\sum_{i=1}^{N-1} \sqrt{\left(x_{i+1}-x_{i}\right)^{2}+\left(y_{i+1}-y_{i}\right)^{2}+\left(z_{i+1}-z_{i}\right)^{2}}
$$

where $i$ enumerates the $2 \mathrm{D}$ slices, and $N$ is the total number of $2 \mathrm{D}$ slices in the 3D microstructure. Geometrical tortuosity $\tau_{\mathrm{g}}$ is finally calculated as the ratio of $L_{\mathrm{g}}$ to $L$, as expressed in Eq. (6).

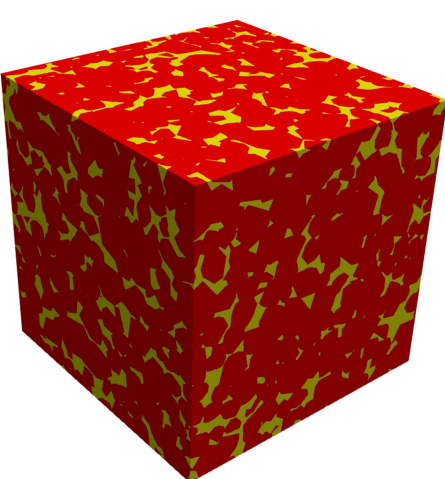

(a)

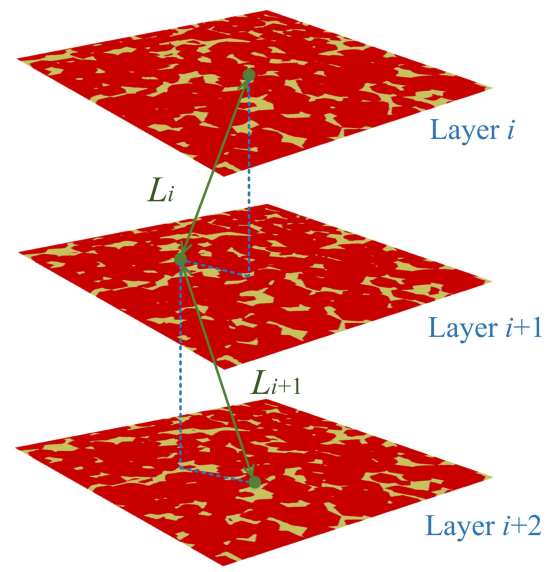

(b)

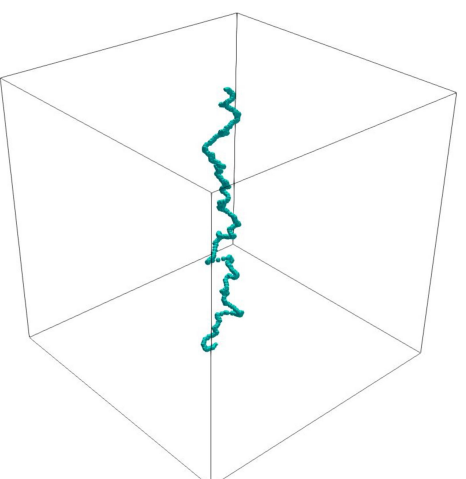

(c)

Figure 7: (a) The 3D digital image of a porous medium; (b) The distances $L_{i}$ between the pore centroids on adjacent 2D slices; (c) The effective pore path from top to bottom surface determined by the PCM.

\section{Physical tortuosities}

In contrast to geometrical tortuosity characterizing the pore microstructure itself, physical tortuosities focus on the transport processes occurring inside the pore microstructure, including transfers of volume, charge, mass and energy. Numerical simulations can be performed on the digital microstructures of porous media to mimic various transport phenomena at the pore scale, thereby to compute corresponding physical tortuosities. 


\subsection{Analogy among different transport phenomena}

The study of transport phenomena in porous media (Adler, 1992; Civan, 2011; Bear and Bachmat, 2012) concerns fluid permeation, electrical conduction, molecular diffusion, heat transfer and momentum exchange, and different transport phenomena share notable commonalities in terms of macroscopic mechanisms. Generally, these transport phenomena are governed by two primary principles at the steady state (Bird, 2002; Brodkey and Hershey, 2003; Plawsky, 2020): (1) the conservation law, which demands the quantity of transport matter (i.e. mass, charge, energy, momentum or others) much be conserved; (2) the constitutive relation, which describes the behavior of transport matter responding to stimuli.

Table 4: The analogy among various transport phenomena in porous media

\begin{tabular}{lllll}
\hline $\begin{array}{l}\text { Transport phenomenon } \\
\text { (Transport matter/quantity) }\end{array}$ & Driving force & $\begin{array}{c}\text { Constitutive } \\
\text { relation }\end{array}$ & $\begin{array}{c}\text { Phenomenological } \\
\text { property }\end{array}$ & Definitive law \\
\hline $\begin{array}{l}\text { Fluid permeation } \\
\text { (Volume) }\end{array}$ & $\begin{array}{l}\text { Hydraulic gradient } \nabla H \\
\text { (Pressure gradient) }\end{array}$ & $\mathbf{J}_{\mathrm{h}}=-K \nabla H$ & $\begin{array}{c}\text { Hydraulic conductivity } K \\
\text { (Intrinsic permeability) }\end{array}$ & Darcy's law \\
$\begin{array}{l}\text { Molecular diffusion } \\
\text { (Mass) }\end{array}$ & Concentrate gradient $\nabla C$ & $\mathbf{J}_{\mathrm{d}}=-D \nabla C$ & Diffusion coefficient $D$ & Fick's law \\
$\begin{array}{l}\text { Electrical conduction } \\
\text { (Charge) }\end{array}$ & Voltage gradient $\nabla V$ & $\mathbf{J}_{\mathrm{e}}=-\sigma \nabla V$ & Electrical conductivity $\sigma$ & Ohm's law \\
$\begin{array}{l}\text { Heat transfer } \\
\text { (Energy) }\end{array}$ & Temperature gradient $\nabla T$ & $\mathbf{J}_{\mathrm{t}}=-\lambda \nabla T$ & Thermal conductance $\lambda$ & Fourier's law \\
$\begin{array}{l}\text { Newtonian viscous flow } \\
\text { (Momentum) }\end{array}$ & Velocity gradient $\nabla U$ & $\mathbf{J}_{\mathrm{m}}=-\mu \nabla U$ & Dynamic viscosity $\mu$ & $\begin{array}{l}\text { Newton's law } \\
\text { of viscosity }\end{array}$ \\
\hline
\end{tabular}

Note: $\mathbf{J}_{\mathrm{h}}, \mathbf{J}_{\mathrm{d}}, \mathbf{J}_{\mathrm{e}}, \mathbf{J}_{\mathrm{t}}$ and $\mathbf{J}_{\mathrm{m}}$ denote fluid flux, diffusion flux, charge flux, heat flux and momentum flux (shear stress) respectively.

For different transport phenomena, if the density of transport matter is constant and independent of time and space, the continuity equation can be simply expressed as follows (Bird, 2002; Brodkey and Hershey, 2003; Plawsky, 2020):

$$
\nabla \cdot \mathbf{J}=0
$$

where $\mathbf{J}$ is the steady-state flux of transport matter. As to the constructive relations, transfers of fluid volume, mass, charge, heat and momentum are almost described identically, which can be seen in Table 4. Different transport processes are governed by similar macroscopic laws (i.e. Darcy's, Fick's, Ohm's, Fourier's and Newton's law), which have a general expression as follows (Bird, 2002; Brodkey and Hershey, 2003; Plawsky, 2020):

$$
\mathbf{J}=-\delta \nabla F_{\text {drive }}
$$

where the steady-state flux $\mathbf{J}$ of transport matter is proportional to the applied driving force $\nabla F_{\text {drive }}$, and the proportionality constant $\delta$ is the phenomenological coefficient corresponding to transport property (i.e. intrinsic permeability, diffusion coefficient, electrical conductivity, thermal conductance or dynamic viscosity).

Massive efforts have been made to study the 'analogy' among various transport phenomena, aiming to achieve the utility of them (Bird, 2002; Brodkey and Hershey, 2003; Plawsky, 2020). Partially due to this mathematical analogy, the physical tortuosities associated with four different transport phenomena (i.e. fluid permeation, electric conduction, molecular diffusion and heat transfer) are often interchangeably used in the literature, without rigorous justification. However, this mathematical analogy in no way means that the physical mechanisms underlying different transport processes are completely equivalent in any aspect. For example, there are discrepancies between the transport streamlines of different transport phenomena happening inside a specific porous medium, which is the primary cause of the inconsistencies between different types of physical tortuosity (more detailed explanation is provided in $\S 4)$. To address this gap, we systematically investigate the hydraulic, electrical, diffusional and thermal tortuosity in the following subsections to clarify their relationships. 


\subsection{Hydraulic tortuosity}

\subsubsection{Definition of hydraulic tortuosity}

Hydraulic tortuosity $\tau_{\mathrm{h}}$ was first proposed by Kozeny (1927) and then further refined by Carman (1937) to model permeability $\kappa$ of porous media through the well-known Kozeny-Carman equation (Clennell, 1997), given by

$$
\kappa=\frac{\phi^{3}}{\beta \tau_{\mathrm{h}}^{2} S^{2}}
$$

where $\phi$ is the porosity defined as the fraction of pore space in the porous medium, $S$ is the specific surface area equal to the ratio of interstitial surface area to bulk volume, and $\beta$ is the shape factor (a constant depending on the type of granular material).

Hydraulic tortuosity $\tau_{\mathrm{h}}$ is defined as (Clennell, 1997):

$$
\tau_{\mathrm{h}}=\frac{L_{\mathrm{h}}}{L}
$$

where $L_{\mathrm{h}}$ denotes the length of effective hydraulic flow path (as illustrated in Figure 2b), and $L$ the straight-line distance through the porous medium in the macroscopic flow direction. The length $L_{\mathrm{h}}$ is usually measured from the streamlines of steady state pore-scale flow. Hydraulic tortuosity $\tau_{\mathrm{h}}$ can be considered as a dimensionless parameter to describe the average elongation of streamlines in a porous medium with respect to the homogeneous flow (Duda et al., 2011). However, the flow flux in a random porous medium varies continuously along the flow path affected by its cross section, shape, orientation, branching and rejoining, making it difficult to identify the streamlines of pore-scale flow.

\subsubsection{Evaluation via fluid flow simulation}

The fluid flow in a digital microstructure can be simulated using such numerical schemes as LBM (Jin et al., 2004) and FVM (Piller et al., 2009), after which hydraulic tortuosity $\tau_{\mathrm{h}}$ can be calculated by analyzing the streamlines determined from the pore-scale simulation. Several methods have been proposed to calculate hydraulic tortuosity $\tau_{\mathrm{h}}$ based on the streamlines. The effective length of hydraulic flow path $L_{\mathrm{h}}$ is often evaluated as a weighted average of streamline lengths (Zhang and Knackstedt, 1995; Duda et al., 2011):

$$
\tau_{\mathrm{h}}=\frac{1}{L}\left(\frac{\sum_{i} w_{i} l_{i}}{\sum_{i} w_{i}}\right)
$$

where $i$ enumerates the separated streamlines, $l_{i}$ is the length of the $i$ th streamline, and $w_{i}$ is the weight of the $i$ th streamline contributing to the overall flux. Different methods have been proposed to determine the weight $w_{i}$. Zhang and Knackstedt (1995) used the travel time $t_{i}$ of a fluid particle moving along the $i$ th streamline to determine the weight $w_{i}$, given by $w_{i}=1 / t_{i}$. Considering the relationship between the travel time and the fluid particle speed along the streamline, Koponen et al. (1996) modified the calculation as follows:

$$
\tau_{\mathrm{h}}=\frac{1}{L} \frac{\int_{\Omega} l(\mathbf{r}) v(\mathbf{r}) \mathrm{d} \Omega}{\int_{\Omega} v(\mathbf{r}) \mathrm{d} \Omega} \approx \frac{1}{L} \frac{\sum_{j} l\left(\mathbf{r}_{j}\right) v\left(\mathbf{r}_{j}\right)}{\sum_{j} v\left(\mathbf{r}_{j}\right)}
$$

where $\Omega$ denotes the volume domain of pore space, $l(\mathbf{r})$ is the length of the streamline passing through a point $\mathbf{r}$, and $v(\mathbf{r})=|\mathrm{v}(\mathbf{r})|$ is the tangential velocity of the fluid at point $\mathbf{r}$. It was later proved by Duda et al. (2011) and Matyka and Koza (2012) that Eq. (15) is equivalent to:

$$
\tau_{\mathrm{h}}=\frac{\int_{\Omega} v(\mathbf{r}) \mathrm{d} \Omega}{\int_{\Omega} v_{x}(\mathbf{r}) \mathrm{d} \Omega}=\frac{\langle v\rangle}{\left\langle v_{x}\right\rangle}
$$

where $v_{x}(\mathbf{r})$ is the velocity component along the macroscopic flow direction at point $\mathbf{r}$, and $\langle\cdot\rangle$ denotes the spatial average over the pore space $\Omega$. Eq. (16) directly calculates hydraulic tortuosity from the steady-state fluid velocity filed, avoiding the difficulty of identifying individual streamlines. This method 
is widely used as it significantly simplifies the calculation of hydraulic tortuosity for porous media with complicated geometries. It should be noted that Eq. (16) is based on two assumptions: the fluid is incompressible and the fluid flow is not reentrant. For laminar flow with a low Reynolds number passing through porous media, the effect of reentrant is negligible.



(a)

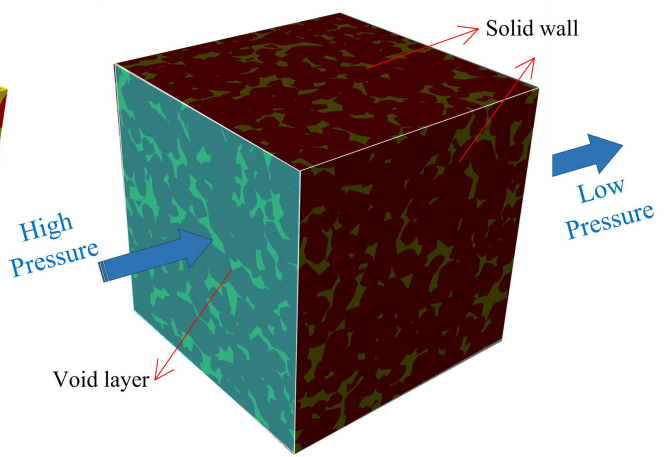

(b)

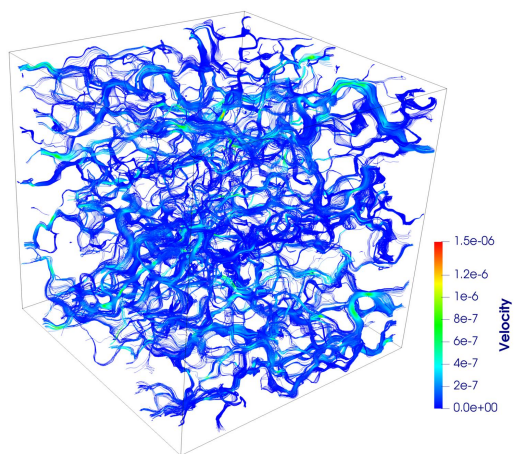

(c)

Figure 8: (a) A 3D porous medium REV; (b) Boundary conditions for the LBM simulation of fluid flow passing through the porous medium REV; (c) The steady-state fluid velocity field inside the porous medium REV.

The LBM is adopted in this work to simulate the single-phase fluid flow passing through digital microstructures, as illustrated in Figure 8c. The LBM models the fluid particles through a timedependent distribution propagating on a regular lattice (Krüger et al., 2017). The pore voxels of digital microstructures serve as the regular lattice, and each lattice node is located in the center of corresponding pore voxel. Starting with an initial state, the particle distribution function moves from one lattice node to its neighboring nodes at each time step, and evolves itself locally subject to both mass and momentum conservation. The local equilibrium particle distribution function corresponds to an ideal state, where the particle distribution tends to a specific macroscopic state, and it is specifically selected to recover the macroscopic Navier-stokes equations. For stationary, creeping, and incompressible Newtonian fluid flow in porous media, Navier-Stokes equations can be simplified to Darcy's law, as listed in Table 4.

Pore-scale simulations using LBM are well established and comprehensively documented in many works of literature (Ginzbourg and Adler, 1994; Moctezuma-Berthier et al., 2004; Jin et al., 2004; Kutay et al., 2006; Fredrich et al., 2006). In this work, the conventional D3Q19 lattice arrangement and Bhatnagar-Gross-Krook (BGK) collision operator are adopted. Since the steady-state flow in porous media is insensitive to the initial state, we simply set the flow velocity to 0 and the fluid density to 1 for the whole domain as the initial conditions. Two types of boundary condition are adopted: the no-slip boundary at the pore-solid interface and the constant pressure boundary at inlet and outlet, as shown in Figure 8b. To apply the constant pressure gradient to the cubic microstructure sample, two void layers are added to the outlet and inlet faces. As to the other four faces that are parallel to the global flow direction, solid walls are added to isolate the cubic REV from the outside. Driven by a constant pressure gradient, the fluid flows from the inlet face towards the outlet face steadily, as illustrated in Figure 8c. The LBM simulation runs iteratively until the steady state is reached, and then hydraulic tortuosity $\tau_{\mathrm{h}}$ can be calculated following Eq. (16).

\subsection{Electrical tortuosity}

\subsubsection{Definition of electrical tortuosity}

Electrical tortuosity is measured through the electrical conduction experiment, where the porous media sample is saturated with an electrolyte of electrical conductivity $\sigma_{0}$, and driven by a voltage difference, the electric charges are transmitted through the pore space filled by the electrolyte liquid (Garrouch et al., 2001; Weerts et al., 2001; Barrande et al., 2007). The effective conductivity $\sigma_{\text {eff }}$ measured on 
the test sample is smaller than the electrolyte conductivity $\sigma_{0}$, due to the retarding effect of porous microstructure on the electric charge transmission (Winsauer et al., 1952). The difference between $\sigma_{0}$ and $\sigma_{\text {eff }}$ is represented by electrical tortuosity $\tau_{\mathrm{e}}$ as follows (Tye, 1983; Patel et al., 2003; Landesfeind et al., 2016):

$$
\sigma_{\text {eff }}=\sigma_{0} \frac{\phi}{\tau_{\mathrm{e}}^{2}}
$$

where $\phi$ is the porosity, and $1 / \tau_{\mathrm{e}}^{2}$ can be understood as the fraction of pore volumes having the same efficiency for electrical conduction as the electrolyte. To be consistent with hydraulic tortuosity $\tau_{\mathrm{h}}$ in Eq. (13)), electrical tortuosity $\tau_{\mathrm{e}}$ was redefined as (Winsauer et al., 1952; Clennell, 1997; Cai et al., 2017):

$$
\tau_{\mathrm{e}}=\frac{L_{\mathrm{e}}}{L}
$$

where $L_{\mathrm{e}}$ is the effective length of the electrical transmission path.

According to the above description, electrical tortuosity $\tau_{\mathrm{e}}$ of a porous medium is defined as a retardation factor of electrical conduction in Eq. (17), and also defined as an elongation factor of electrical transmission path in Eq. (18). However, the equivalence between these two definitions is derived from a simple capillary tube model (Barrande et al., 2007), while it remains unclear whether or not a similar correlation holds for general porous media (Duda et al., 2011).

\subsubsection{Evaluation via electrical conduction simulation}

Electrical conduction in porous media can be simulated by various numerical methods such as LBM (Sun and Wong, 2018), FEM (Saomoto and Katagiri, 2015), FDM (Schwartz et al., 1995) and RWM (Ioannidis et al., 1997). In this work, we use the Avizo package (Avizo, 2017) to simulate electric charges transmitting through 3D digital microstructures, and the electrical tortuosity is then calculated according to Eq. (17). The Avizo solver is based FVM (Moukalled et al., 2016), where the image voxels are directly used as the volume elements. The solid phase is assumed to be homogeneous and insulating, and the pore space is saturated with an electrolyte of electrical conductivity $\sigma_{0}$. As shown in Figure 9b, a constant electrical potential difference is applied on the inlet and outlet faces of the cubic digital microstructure, while the other four faces are set as insulating. The boundary condition for the pore-solid interface is set as follows:

$$
\nabla V \cdot \mathbf{n}=0
$$

where $\mathbf{n}$ is the unit vector normal to the pore-solid surface, pointing to the solid phase.



(a)

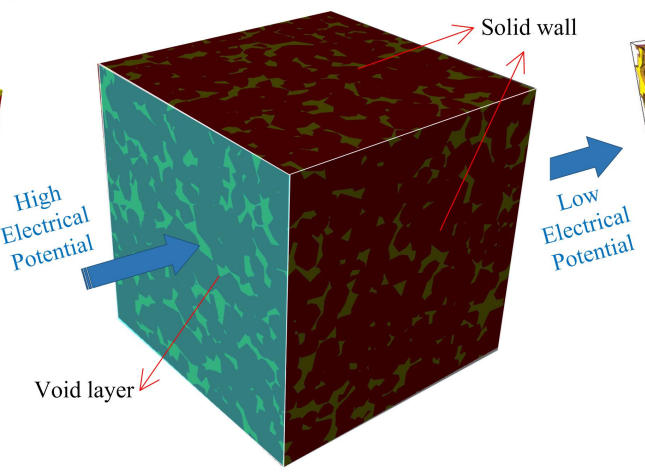

(b)

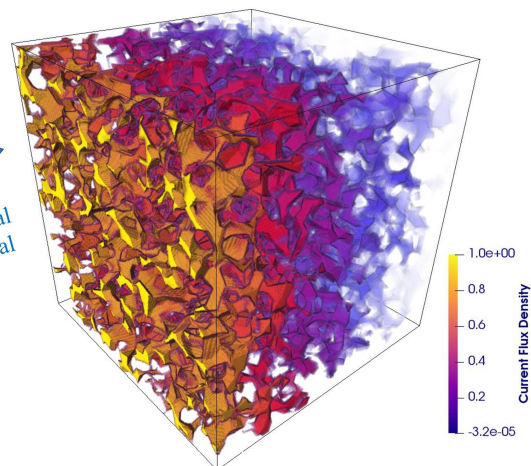

(c)

Figure 9: (a) A 3D porous medium REV; (b) Boundary conditions for the FVM simulation of electrical conduction in the porous medium REV; (c) The steady-state electrical flux density inside the porous medium REV.

Driven by the constant electric potential gradient, electric charges are transmitted through the porous media sample filled with electrolyte. The electrical conduction at the steady state is governed 
by the charge conservation and Ohm's law, as expressed in Eq. (10) and Eq. (11) respectively. The effective (apparent) electrical conductivity $\sigma_{\text {eff }}$ can be estimated by

$$
\frac{J_{\mathrm{e}}^{(\text {Pore })}}{A}=\sigma_{\mathrm{eff}} \frac{V_{\text {outlet }}-V_{\text {inlet }}}{L}
$$

where $J_{\mathrm{e}}^{(\text {Pore })}$ is the electrical flux passing through the outlet face of the porous media sample, $A$ is the cross section area perpendicular to the flow direction, $V_{\text {inlet }}$ and $V_{\text {outlet }}$ are the applied electrical potentials at the inlet and outlet faces respectively, and $L$ is the distance between inlet and outlet faces. The total electrical flux $J_{\mathrm{e}}^{(\text {Pore })}$ can be obtained through an integration of local electrical flux, given by

$$
J_{\mathrm{e}}^{(\text {Pore })}=\int_{A}-\sigma_{0} \nabla V \mathrm{~d} A
$$

Substituting Eq. (20) and Eq. (21) into Eq. (17), electrical tortuosity $\tau_{\mathrm{e}}$ can be computed as:

$$
\tau_{\mathrm{e}}=\sqrt{\frac{\sigma_{0}}{\sigma_{\mathrm{eff}}} \phi}=\sqrt{\frac{A \sigma_{0} \frac{V_{\text {outlet }}-V_{\text {inlet }}}{L}}{J_{\mathrm{e}}^{(\text {Pore })}}} \phi=\sqrt{\frac{J_{\mathrm{e}}^{(\text {Free })}}{J_{\mathrm{e}}^{(\text {Pore })}} \phi}
$$

where $J_{\mathrm{e}}^{(\text {Free })}$ is the electrical flux passing through a homogeneous medium with the same dimensions, of the electrical conductivity $\sigma_{0}$, and driven by the same electrical potential gradient. As discussed earlier, electrical tortuosity $\tau_{\mathrm{e}}$ is often used to substitute hydraulic tortuosity $\tau_{\mathrm{h}}$ whose direct evaluation is much more time-consuming and costly.

\subsection{Diffusional tortuosity}

\subsubsection{Definition of diffusional tortuosity}

The tortuosity of a porous medium can also be measured from the diffusion experiment, where diffusing gases are typically used as the probe due to their high diffusion rates (Van Brakel and Heertjes, 1974; Kreamer et al., 1988). The effective diffusivity $D_{\text {eff }}$ in a porous medium is lower than the bulk diffusivity $D_{0}$ of the diffusing gas (Garrouch et al., 2001). Diffusional tortuosity $\tau_{\mathrm{d}}$ is determined from the ratio of $D_{0}$ to $D_{\text {eff }}$ (Van Brakel and Heertjes, 1974; Epstein, 1989; Boudreau, 1996; Moldrup et al., 2001; Tjaden et al., 2016), given by

$$
D_{\text {eff }}=D_{0} \frac{\phi \delta}{\tau_{\mathrm{d}}^{2}}
$$

where $\delta$ is the constriction coefficient due to the variation of pore diameter along the diffusion pathway, and its value also depends on the ratio of the solute diameter (probe molecule dimension) to the pore diameter. The kinetic diameter of probe gas molecule is usually smaller than $1 \mathrm{~nm}$ (Sing and Williams, 2004) and when the pore diameter is larger than $1 \mu \mathrm{m}, \delta$ is approximately 1 (Grathwohl, 2012). By modeling the porous medium with cylindrical capillaries, diffusional tortuosity $\tau_{\mathrm{d}}$ was also defined as the ratio between the effective length of diffusive path $L_{\mathrm{d}}$ and the straight-line distance $L$ (Epstein, 1989), given by

$$
\tau_{\mathrm{d}}=\frac{L_{\mathrm{d}}}{L}
$$

Generally speaking, mass diffusion inside porous media may involve ordinary molecular (Fickian) diffusion (Hosticka et al., 1998) and Knudsen diffusion (Malek and Coppens, 2003), depending on the ratio of the mean free path of the probe molecules to the pore diameter. If the mean free path is relatively short compared to the pore size, only the molecular diffusion occurs, and the diffusion process can be described by Fick's law, as listed in Table 4. Knudsen diffusion takes place when the mean free path is comparable to the pore size, where the molecules collide with the pore walls more frequently than with each other, impeding the molecule transport. The diffusion transport usually decreases as the 
probe molecule size increases, because more collisions occur between the molecules and the pore walls. Therefore, the tortuosity results obtained from different diffusion measurements may differ, depending on the diffusion regime and the probe molecule. The thresholds of pore size are $1 \mu \mathrm{m}$ and $10 \mathrm{~nm}$ for Fickian diffusion and Knudsen diffusion respectively (Wyllie and Spangler, 1952; Garrouch et al., 2001). That is, the Knudsen effect is negligible when pore size is larger than $1 \mu \mathrm{m}$, and in this case the diffusion tortuosity is independent of the probe molecule. In this work, only the ordinary molecular diffusion is considered and diffusional tortuosity $\tau_{\mathrm{d}}$ is evaluated according to Eq. (23), where the constriction coefficient $\delta$ is set as 1 .

\subsubsection{Evaluation via molecular diffusion simulation}

The molecular diffusion process in porous media can be simulated by such numerical methods as FVM (Tjaden et al., 2018), LBM (Ostadi et al., 2010) and FDM (Cooper, 2015). In this work, we adopt the open-source solver TauFactor (Cooper et al., 2016), which is based on FDM and directly uses image voxels as the discretization mesh for simulation. As shown in Figure 10, diffusional tortuosity is calculated by comparing the steady-state diffusive fluxes passing through the cubic porous medium REV and the cubic homogeneous REV with the same dimensions. A constant concentration difference is applied between inlet and outlet faces of both REVs, while the other four faces parallel to the diffusional flow direction are set as solid walls for isolation purpose. The boundary condition at the pore-solid interface is set as:

$$
\nabla C \cdot \mathbf{n}=0
$$

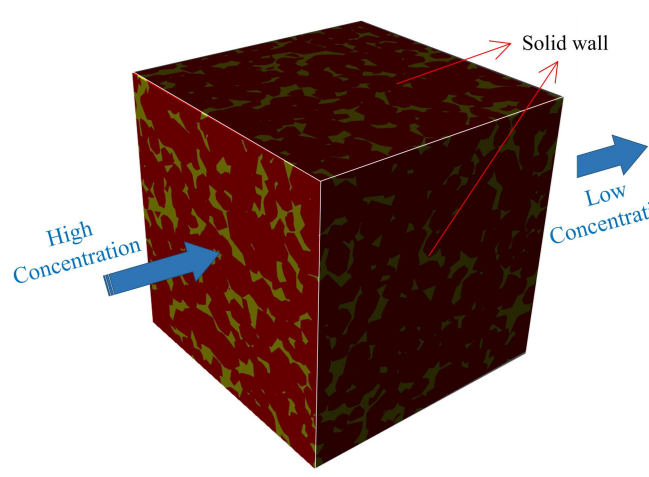

(a)

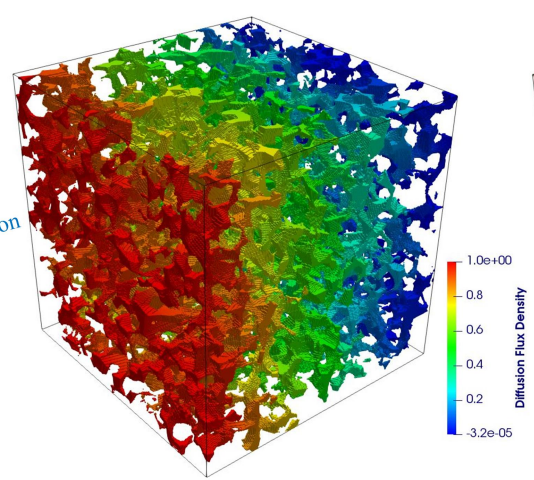

(b)

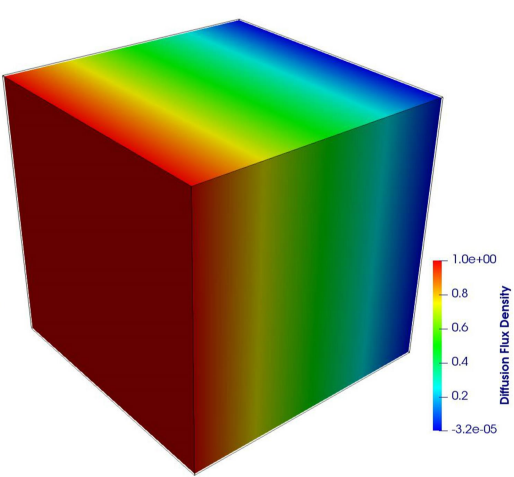

(c)

Figure 10: (a) The schematic diagram of boundary conditions for the FDM simulation of molecular diffusion in a porous medium REV; (b) The diffusional flux density at the steady state inside the porous medium REV; (c) The diffusional flux density at the steady state inside the homogeneous REV.

Driven by the constant concentration gradient, the diffusive specie moves from the inlet face to the outlet face. When the steady state is reached, the diffusional flux passing through the porous media REV and the homogeneous REV can be obtained from Fick's law:

$$
\begin{aligned}
J_{\mathrm{d}}^{(\text {Pore })} & =-A D_{\text {eff }} \frac{\Delta C}{L} \\
J_{\mathrm{d}}^{(\text {Free })} & =-A D_{0} \frac{\Delta C}{L}
\end{aligned}
$$

where $J_{\mathrm{d}}^{(\text {Pore })}$ and $J_{\mathrm{d}}^{(\text {Free })}$ are the diffusion fluxes passing through the porous media REV and the homogeneous REV, respectively. Taking the ratio of $J_{\mathrm{d}}^{(\text {Pore) }}$ to $J_{\mathrm{d}}^{\text {(Free) }}$ and then rearranging the equation, diffusional tortuosity $\tau_{\mathrm{d}}$ can be expressed as:

$$
\tau_{\mathrm{d}}=\sqrt{\frac{D_{0}}{D_{\text {eff }}} \phi}=\sqrt{\frac{J_{\mathrm{d}}^{\text {(Free })}}{J_{\mathrm{d}}^{\text {(Pore })}} \phi}
$$




\subsubsection{Evaluation via random walk simulation}

Diffusional tortuosity $\tau_{\mathrm{d}}$ of a porous medium can also be statistically estimated from the random walk process of non-absorbing particles, which can be considered as a simplified diffusion simulation (Salles et al., 1993; Nakashima and Watanabe, 2002; Nakashima and Kamiya, 2007; Iwai et al., 2010; Hu et al., 2012; Tjaden et al., 2018). The random walk simulation starts by randomly distributing a number of walkers into the pore space at $t=0$. Then, each walker randomly selects one of its neighboring voxels (e.g. following the 6-neighbor-connectivity criterion) as its possible position at the next time step. If the selected voxel belongs to the pore phase, the walker moves to the new position; otherwise the walker stays at the current location. With the random walking procedure repeated, the mean square displacement $\left\langle r^{2}(t)\right\rangle$ of all random walkers is a function of time step $t$ :

$$
\left\langle r^{2}(t)\right\rangle=\frac{1}{N} \sum_{i=1}^{N}\left\{\left[x_{i}(t)-x_{i}(0)\right]^{2}+\left[y_{i}(t)-y_{i}(0)\right]^{2}+\left[z_{i}(t)-z_{i}(0)\right]^{2}\right\}
$$

where $N$ is the number of random walkers, $x_{i}(t), y_{i}(t)$ and $z_{i}(t)$ are the coordinates of the $i$ th walker at time $t$, and $\langle\cdot\rangle$ denotes the ensemble average.

For random walkers walking inside a cubic homogeneous REV, the mean square displacement $\left\langle r^{2}(t)\right\rangle_{\text {Free }}$ of them is related to the diffusion coefficient $D_{\text {Free }}$ as follows:

$$
D_{\text {Free }}=\frac{1}{6} \frac{\mathrm{d}\left\langle r^{2}(t)\right\rangle_{\text {Free }}}{\mathrm{d} t}
$$

where $D_{\text {Free }}$ is the diffusion coefficient in the cubic homogeneous REV (e.g. the diffusivity of gas or water). While for random walkers in a porous medium REV, the mean square displacement $\left\langle r^{2}(t)\right\rangle_{\text {Pore }}$ of them is reduced due to the obstruction effect from solid phase, which is related to the effective diffusion coefficient $D_{\text {Pore }}$ as follows:

$$
D_{\text {Pore }}=\phi \frac{1}{6} \frac{\mathrm{d}\left\langle r^{2}(t)\right\rangle_{\text {Pore }}}{\mathrm{d} t}
$$

$D_{\text {Free }}$ is a constant because of the unrestricted diffusion in the cubic homogeneous REV, but $D_{\text {pore }}$ is time-dependent due to local heterogeneity present in porous media. The random walk trajectory is restricted by the solid phase, thereby reducing the diffusivity.

Diffusional tortuosity $\tau_{\mathrm{d}}$ is calculated from the ratio of $D_{\text {Free }}$ to $D_{\text {Pore }}$ as follows:

$$
\tau_{\mathrm{d}}=\sqrt{\frac{D_{\text {Free }}}{D_{\text {Pore }}}} \phi=\sqrt{\frac{\mathrm{d}\left\langle r^{2}(t)\right\rangle_{\text {Free }} / \mathrm{d} t}{\mathrm{~d}\left\langle r^{2}(t)\right\rangle_{\text {Pore }} / \mathrm{d} t}}
$$

Long-time data of the mean square displacement $\left\langle r^{2}(t)\right\rangle$ from large numbers of random walkers can fully experience the porous microstructure, and the slope $\mathrm{d}\left\langle r^{2}(t)\right\rangle_{\text {Pore }} / \mathrm{d} t$ approximates to a constant value, as shown in Figure 11c.

A natural porous medium such as rock often possesses an anisotropic pore structure. If the porous medium is significantly anisotropic, the effective diffusivity $D_{\text {Pore }}$ is tensor instead of a scalar, and Eqs. (29) to (32) break down (Nakashima and Kamiya, 2007). Directional mean square displacements $\left\langle x^{2}(t)\right\rangle,\left\langle y^{2}(t)\right\rangle$ and $\left\langle z^{2}(t)\right\rangle$ are required to evaluate the directional tortuosities of the anisotropic porous medium (Nakashima and Kamiya, 2007; Iwai et al., 2010), given by

$$
\begin{aligned}
\left\langle x^{2}(t)\right\rangle_{\text {Pore }} & =\frac{1}{N} \sum_{i=1}^{N}\left[x_{i}(t)-x_{i}(0)\right]^{2} \\
\left\langle y^{2}(t)\right\rangle_{\text {Pore }} & =\frac{1}{N} \sum_{i=1}^{N}\left[y_{i}(t)-y_{i}(0)\right]^{2}
\end{aligned}
$$




$$
\begin{gathered}
\left\langle z^{2}(t)\right\rangle_{\text {Pore }}=\frac{1}{N} \sum_{i=1}^{N}\left[z_{i}(t)-z_{i}(0)\right]^{2} \\
\left\langle x^{2}(t)\right\rangle_{\text {Free }}=\left\langle y^{2}(t)\right\rangle_{\text {Free }}=\left\langle z^{2}(t)\right\rangle_{\text {Free }}=\frac{1}{3}\left\langle r^{2}(t)\right\rangle_{\text {Free }}
\end{gathered}
$$

Then, the directional tortuosities $\tau_{x}, \tau_{y}$ and $\tau_{z}$ can be calculated as follows:

$$
\begin{gathered}
\tau_{x}=\sqrt{\frac{\mathrm{d}\left\langle x^{2}(t)\right\rangle_{\text {Free }} / \mathrm{d} t}{\mathrm{~d}\left\langle x^{2}(t)\right\rangle_{\text {Pore }} / \mathrm{d} t}} \\
\tau_{y}=\sqrt{\frac{\mathrm{d}\left\langle y^{2}(t)\right\rangle_{\text {Free }} / \mathrm{d} t}{\mathrm{~d}\left\langle y^{2}(t)\right\rangle_{\text {Pore }} / \mathrm{d} t}} \\
\tau_{z}=\sqrt{\frac{\mathrm{d}\left\langle z^{2}(t)\right\rangle_{\text {Free }} / \mathrm{d} t}{\mathrm{~d}\left\langle z^{2}(t)\right\rangle_{\text {Pore }} / \mathrm{d} t}}
\end{gathered}
$$

In our implementation, the random walk simulation lasts for 1,000,000 time steps with 10,000 random walkers, and the periodic boundary condition is set to avoid the random walkers moving out the cubic REV sample with a finite size. Data obtained from the first 500,000 time steps are dismissed, because the random walkers have not fully experienced the porous microstructure at the early stage of the simulation.

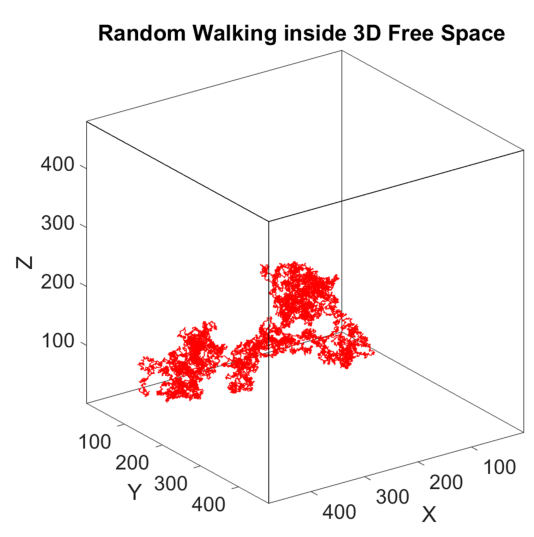

(a)

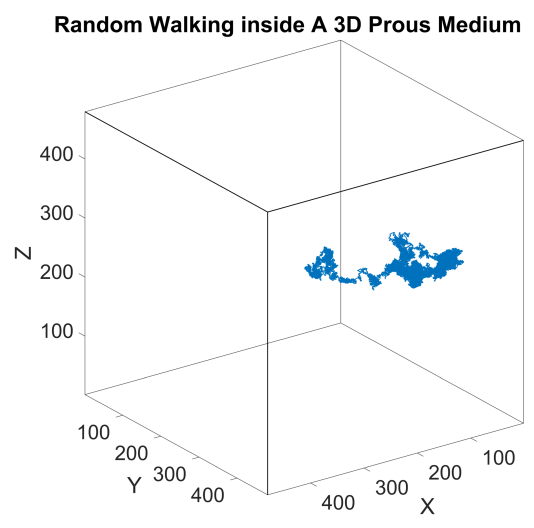

(b)

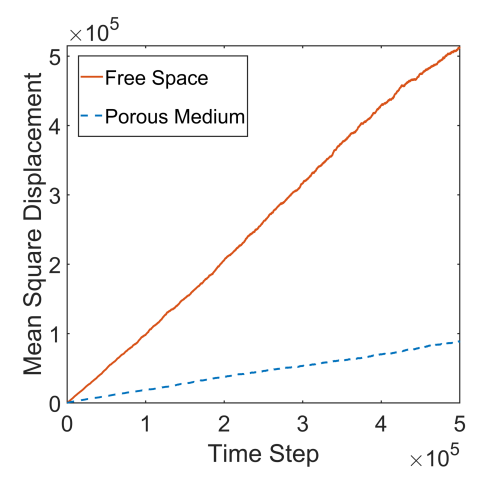

(c)

Figure 11: (a) The trajectory of a single random walker moving inside the free space for 1,000,000 time steps; (b) The trajectory of a single random walker moving inside the pore space for 1,000,000 time steps; (c) The mean square displacements of the random walking averaged over 10,000 walkers.

\subsection{Thermal tortuosity}

\subsubsection{Definition of thermal tortuosity}

Heat transfer in a porous medium is mainly determined by the solid phase conduction. The convective and radiative effects can be largely ignored (Olives and Mauran, 2001), because the thermal conductivity of solid matrix is usually much higher than that of the void phase of air. Therefore, the concept of thermal tortuosity is sometimes related to the winding paths of heat flow through the solid phase of a porous medium (Olives and Mauran, 2001; Bodla et al., 2010). However, this work focuses on the tortuosity of pore space, and as such we simulate the heat conduction passing through the void phase of the porous medium, by assuming the solid phase to be thermal insulating (Moctezuma-Berthier et al., 
2002). The effective thermal conductivity $\lambda_{\text {eff }}$ can be evaluated from the heat flux simulation, based on which the thermal tortuosity $\tau_{\mathrm{t}}$ of pore space can be obtained as follows (Cooper et al., 2013, 2014):

$$
\lambda_{\text {eff }}=\lambda_{0} \frac{\phi}{\tau_{\mathrm{t}}^{2}}
$$

where $\lambda_{0}$ is the bulk thermal conductivity of air.

\subsubsection{Evaluation via heat transfer simulation}

Heat transfer through the pore space can be numerically simulated by using FVM (Cooper et al., 2014), FDM (Wiegmann and Zemitis, 2006) and LBM (Guo and Zhao, 2005). The FVM-based Avizo package (Avizo, 2017) is adopted in this work to simulate the heat flux passing through porous media, and the thermal tortuosity is calculated according to Eq. (40). The pore space is fully filled with air of thermal conductivity $\lambda_{0}$, while the solid phase is assumed to be homogeneous and thermal insulating. A constant temperature difference is applied between input and output faces of the cubic porous media REV, and the other faces are set as thermal insulating walls. The boundary condition on the pore-solid interface is set as follows:

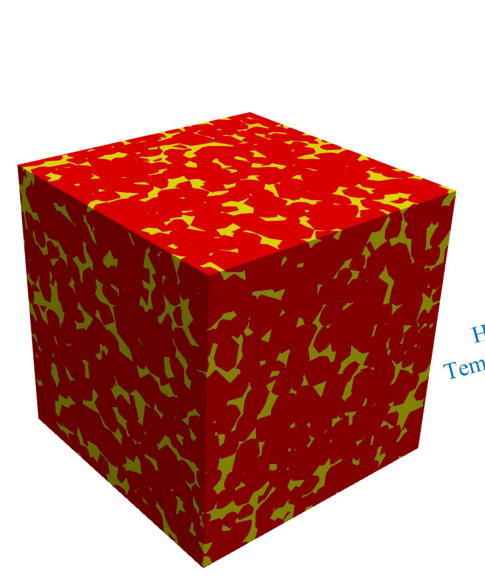

(a)

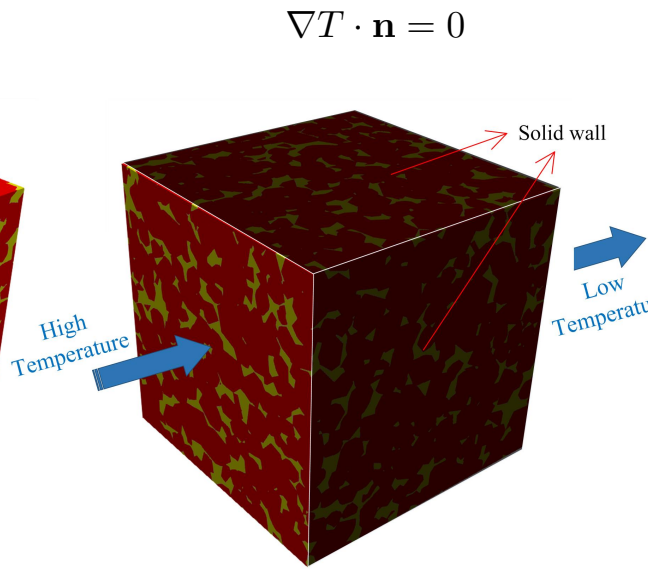

(b)



(c)

Figure 12: (a) A 3D porous medium REV; (b) Boundary conditions of the FVM simulation of heat flux passing through the porous medium REV; (c) The heat flux density inside the porous medium REV at the steady state.

Driven by the temperature difference, heat transfers inside the pore space from the higher temperature area to the lower temperature area. When the steady-state is reached, the heat flow can be described by the conservation of energy in Eq. (10) and Fourier's law in Table 4. The effective (apparent) thermal conductivity $\lambda_{\text {eff }}$ can be calculated by:

$$
\frac{J_{\mathrm{t}}^{(\text {Pore })}}{A}=\lambda_{\text {eff }} \frac{T_{\text {outlet }}-T_{\text {inlet }}}{L}
$$

where $J_{\mathrm{t}}^{(\text {Pore })}$ is the heat flux passing through the output face of the cubic porous medium REV sample, and $T_{\text {intlet }}$ and $T_{\text {outlet }}$ are the applied temperature at the input and output faces, respectively. The total heat flux $J_{\mathrm{t}}^{\text {(Pore) }}$ can be obtained by integrating the local heat flux computed from Fourier's law:

$$
J_{\mathrm{t}}^{(\text {Pore })}=\int_{A}-\lambda_{0} \nabla T \mathrm{~d} A
$$

Substituting Eq. (42) and Eq. (43) into Eq. (40), thermal tortuosity $\tau_{\mathrm{t}}$ can be computed as:

$$
\tau_{\mathrm{t}}=\sqrt{\frac{\lambda_{0}}{\lambda_{\text {eff }}} \phi}=\sqrt{\frac{A \lambda_{0} \frac{T_{\text {outlet }}-T_{\text {inlet }}}{L}}{J_{\mathrm{t}}^{(\text {Pore })}}} \phi=\sqrt{\frac{J_{\mathrm{t}}^{(\text {Free })}}{J_{\mathrm{t}}^{(\text {Pore })}} \phi}
$$


where $J_{t}^{(\text {Free })}$ is the heat flux transferring through a cubic homogeneous REV filled with air of thermal conductivity $\lambda_{0}$, with the same dimensions as the porous medium REV, and driven by the same temperature gradient.

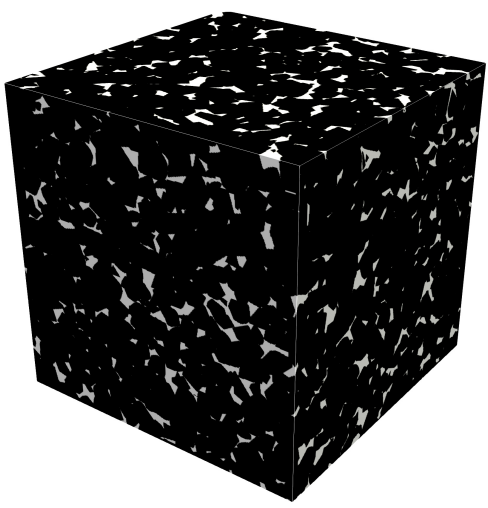

(a) $\phi=8.61 \%$

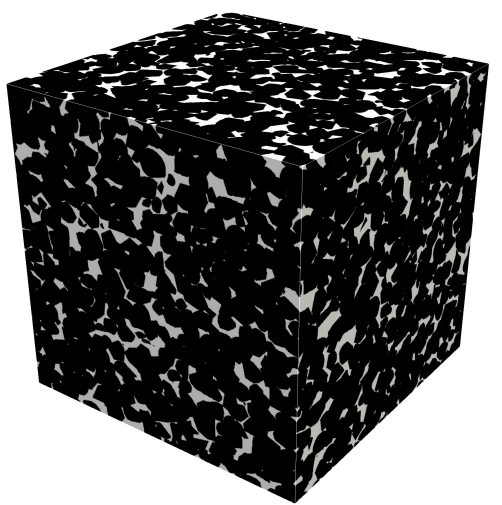

(d) $\phi=15.36 \%$

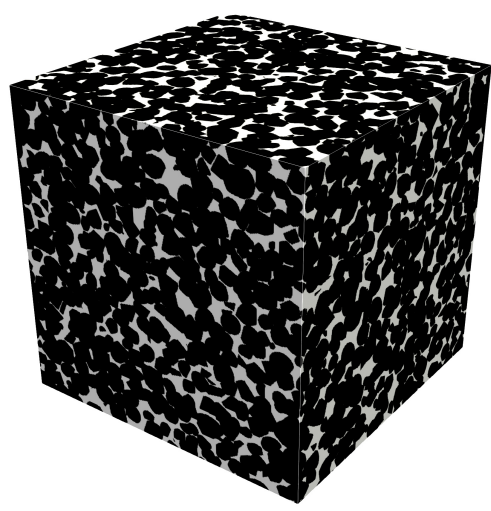

(f) $\phi=20.65 \%$

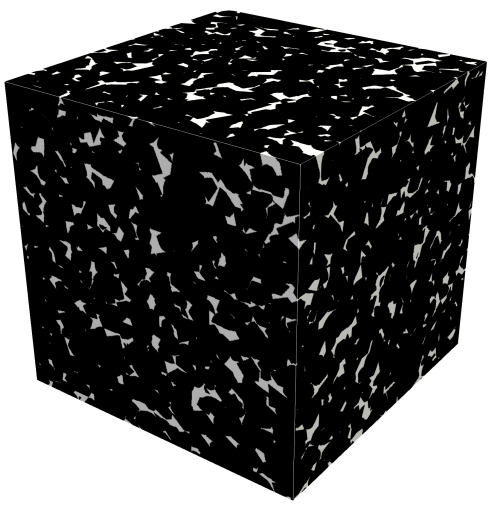

(b) $\phi=10.15 \%$

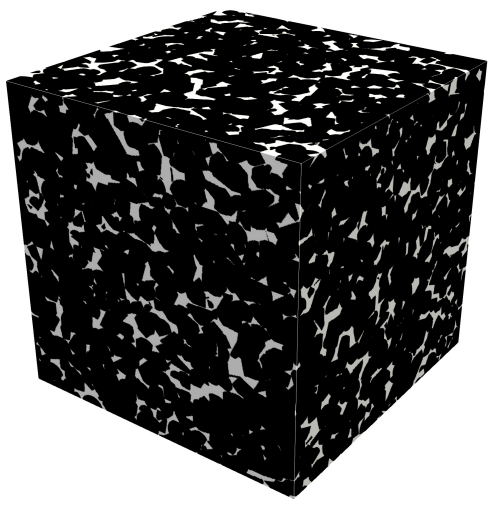

(c) $\phi=12.57 \%$

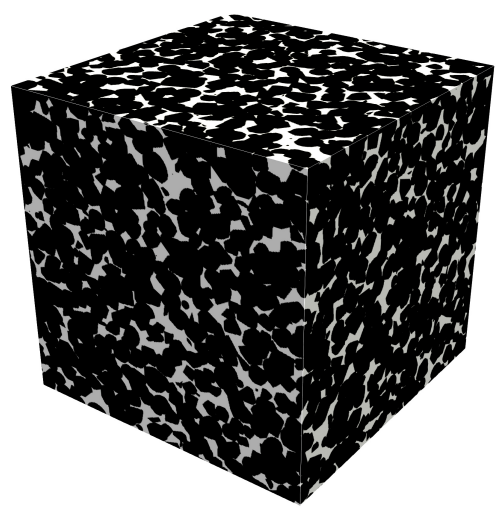

(e) $\phi=17.62 \%$

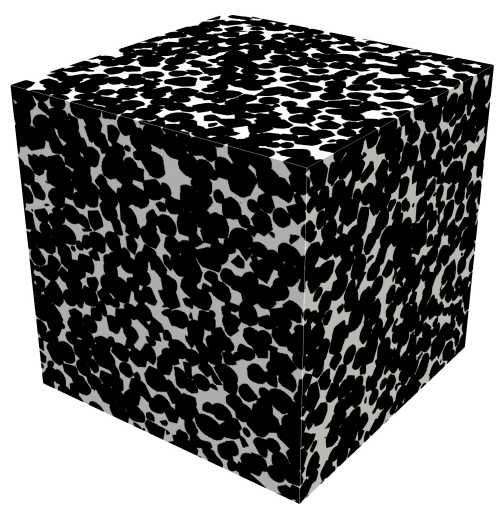

(g) $\phi=24.50 \%$

Figure 13: The 3D digital microstructures of Fontainebleau sandstone samples with different porosities $\phi$ (pore space is shown in white, and solid matrix is shown in black).

\section{Comparison and analysis}

A set of Fontainebleau sandstones with porosity ranging from $8.61 \%$ to $24.50 \%$ are used to compare the tortuosity results evaluated from different image analysis methods, physical simulations and porositytortuosity models. As shown in Figure 13, the 3D REV samples (in binary forms) are of $480 \times 480 \times 480$ voxels with a image resolution of $5.700 \mu \mathrm{m}$, representing an equivalent physical sample of $2.7 \mathrm{~mm}^{3}$ cube 
(Berg, 2016). For each REV sample, both geometrical and physical tortuosities are computed for three principal directions using the methods reviewed in $\S 2$ and $\S 3$, respectively. Fontainebleau sandstone is considered isotropic and to minimize the sampling error, a characteristic tortuosity $\tau$ is obtained by averaging the directional tortuosities (Cooper et al., 2014):

$$
\frac{1}{\tau^{2}}=\frac{1}{3}\left(\frac{1}{\tau_{x}^{2}}+\frac{1}{\tau_{y}^{2}}+\frac{1}{\tau_{z}^{2}}\right)
$$

where $\tau_{x}, \tau_{y}$ and $\tau_{z}$ are tortuosities in the $x, y$ and $z$ direction respectively.

The tortuosity results evaluated from different methods for the set of Fontainebleau sandstone are summarized in Table 5 and plotted in Figure 14. Although the overall trends of tortuosity in relation to porosity are similar, remarkable discrepancies can be observed across different methods, especially for the samples with low porosity.

Table 5: Geometrical and physical tortuosities of the Fontainebleau sandstone samples.

\begin{tabular}{|c|c|c|c|c|c|c|c|c|}
\hline \multirow{2}{*}{ Tortuosity type } & \multirow{2}{*}{ Calculation method/model } & \multicolumn{7}{|c|}{ Tortuosity values of samples with different porosities } \\
\hline & & $8.61 \%$ & $10.15 \%$ & $12.57 \%$ & $15.36 \%$ & $17.62 \%$ & $20.65 \%$ & $24.50 \%$ \\
\hline Geometrical & DSPSM & 1.91 & 1.76 & 1.57 & 1.46 & 1.40 & 1.34 & 1.28 \\
\hline Geometrical & SSPSM & 2.07 & 1.98 & 1.83 & 1.71 & 1.65 & 1.63 & 1.61 \\
\hline Geometrical & FMM & 1.54 & 1.32 & 1.20 & 1.13 & 1.11 & 1.07 & 1.05 \\
\hline Geometrical & PCM & 2.97 & 2.79 & 2.41 & 2.39 & 2.15 & 2.03 & 1.84 \\
\hline Hydraulic & LBM simulation & 2.02 & 1.95 & 1.81 & 1.67 & 1.59 & 1.52 & 1.45 \\
\hline Electrical & FVM simulation & 4.32 & 3.50 & 2.90 & 2.29 & 2.04 & 1.82 & 1.65 \\
\hline Diffusional & FDM simulation & 4.33 & 3.51 & 2.85 & 2.29 & 2.04 & 1.82 & 1.65 \\
\hline Diffusional & RWM simulation & 4.30 & 3.49 & 2.90 & 2.36 & 2.08 & 1.85 & 1.73 \\
\hline Thermal & FVM simulation & 4.33 & 3.50 & 2.91 & 2.29 & 2.04 & 1.82 & 1.65 \\
\hline Diffusional & $\begin{array}{l}\text { Bruggeman relation } \\
\qquad \alpha=1.5)\end{array}$ & 1.85 & 1.77 & 1.68 & 1.60 & 1.54 & 1.48 & 1.42 \\
\hline Hydraulic & $\begin{array}{l}\text { Bruggeman relation } \\
\qquad(\alpha=1.8)\end{array}$ & 2.67 & 2.50 & 2.29 & 2.12 & 2.00 & 1.88 & 1.76 \\
\hline Electrical & $\begin{array}{c}\text { Archie's law } \\
(\alpha=2.5)\end{array}$ & 6.29 & 5.56 & 4.74 & 4.08 & 3.68 & 3.26 & 2.87 \\
\hline Hydraulic & $\begin{array}{l}\text { Comiti's model } \\
\quad(p=0.49)\end{array}$ & 1.48 & 1.46 & 1.42 & 1.38 & 1.36 & 1.33 & 1.30 \\
\hline Diffusional & $\begin{array}{l}\text { Iversen's model } \\
\qquad(q=2)\end{array}$ & 1.68 & 1.67 & 1.66 & 1.64 & 1.63 & 1.61 & 1.58 \\
\hline Hydraulic & Du Plessis's model & 1.48 & 1.47 & 1.47 & 1.46 & 1.45 & 1.45 & 1.43 \\
\hline Geometrical & $\begin{array}{l}\text { Lanfrey's model } \\
\quad(\xi=0.9)\end{array}$ & 15.64 & 12.97 & 10.10 & 7.92 & 6.66 & 5.40 & 4.26 \\
\hline
\end{tabular}

\subsection{Comparison between geometrical tortuosity results}

The geometrical tortuosities of Fontainebleau sandstone samples are computed using four image-based methods, as reviewed in $\S 2$. It can be observed in Table 5 and Figure 14 that remarkable differences are present between the tortuosity results, although the trends in relation to porosity are similar. The largest and smallest geometrical tortuosity results are produced by the PCM and the FMM, respectively, and the difference between them exceeds $75 \%$. The DSPSM and the SSPSM provide similar geometrical tortuosities, due to the same searching algorithm (Dijkstra's algorithm) being used. Because of the difference in image preparation, the tortuosity results from the DSPSM are usually smaller than that calculated from the SSPSM, as discussed in $\S 2.3$. Tortuosity results obtained from the FMM are always smaller than that calculated from the DSPSM and the SSPSM, which means the shortest pore channels identified by the FMM are 'shorter' than that searched by Dijkstra's algorithm. A stable difference between the geometrical tortuosity results computed from the FMM and Dijkstra's algorithm can be observed. As to the PCM, it is more suitable for the single pore channel case, where it recognizes the central axis of pore channel as the effective flow path.

Several factors could affect image analysis results, thereby bringing uncertainties to the computation of geometrical tortuosity: (1) Image preparation. For instance, the DSPSM directly searches for the 


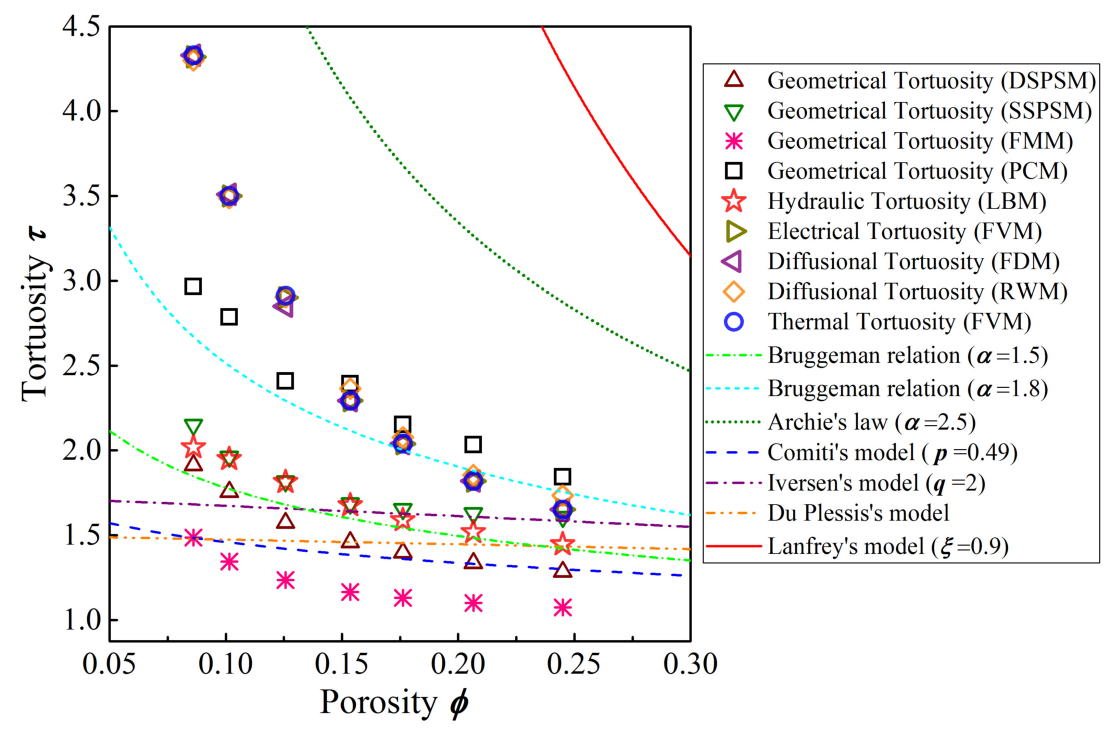

Figure 14: The tortuosity results calculated from different image analysis methods, physical simulations and porosity-tortuosity models for the set of Fontainebleau sandstone samples.

shortest pore channel within the raw pore space, while the SSPSM operates on the pore skeleton after image skeletonization, leading to inconsistent results of geometrical tortuosity between them. The adopted skeletonization algorithm can also affect the extracted topology of the 3D graph (Holzer et al., 2013) and then influence the tortuosity calculation. (2) The definition of the effective flow path $L_{\mathrm{g}}$. The shortest pore channel $L_{\min }$ is often taken as the effective flow path through the complex pore microstructure (Lindquist et al., 1996), but different criteria have also been used by other methods such as the PCM. (3) The path searching algorithm and the definition of pixel/voxel connectivity. For examples, the shortest pore channel searched by the fast marching method is different from the one obtained by Dijkatra's algorithm, and the shortest path based on the 6-neighbor-connectivity criterion (as shown in Figure 15) is usually longer than that based on the 18- or 26-neighbor-connectivity criterion. (4) The distance metric. A number of distance metrics exist for regular image grids, among which Manhattan distance, Euclidean distance and Chebyshev distance are widely used (Chen-Wiegart et al., 2014). These distance metrics yield different distances between voxels, as shown in Figure 16. The choice of distance metric directly affects the identification of the shortest/effective pore channel, thereby affecting the geometrical tortuosity result.

What's more, the effects coming from image resolution and image size on tortuosity computation cannot be ignored, but they can be reduced/avoided by using the digital REV samples with sufficient high resolutions and large sizes, which will be further discussed in $\S 6$. With the above intrinsic uncertainties present in various image-based calculation methods, proper use of geometrical tortuosity requires a standard procedure, clear definition and reasonable parameter selection, to ensure reproducible and portable results.

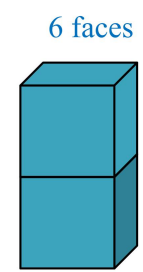

(a) Sharing a face

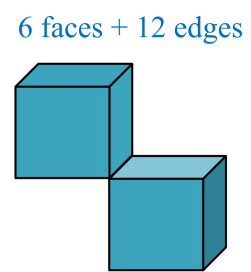

(b) Sharing an edge

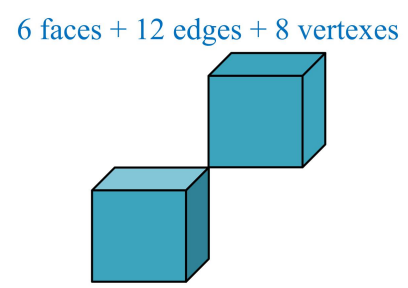

(c) Sharing a vertex

Figure 15: Different connectivity criteria between adjacent image voxels: (a) 6-neighbor-connectivity; (b) 18-neighbor-connectivity; (c) 26-neighbor-connectivity. 


$$
d=\left(\sum_{i=1}^{n}\left|x_{i}-y_{i}\right|^{1 / p}\right)^{p}
$$

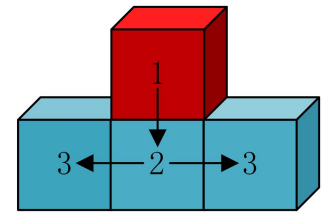

(a) $p=1$

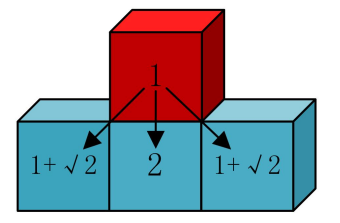

(b) $p=2$

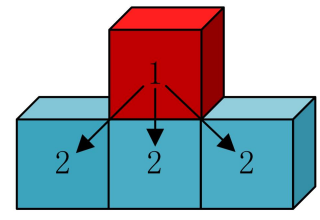

(c) $p=\infty$

Figure 16: Different distance metrics between image voxels: (a) Manhattan distance; (b) Euclidean distance; (c) Chebyshev distance.

\subsection{Comparison between physical tortuosity results}

The physical tortuosities of the set of Fontainebleau sandstone samples are computed via different physical simulations as discussed in $\S 3$, and the results are recorded in Table 5 and plotted in Figure 14. It is observed that the diffusional, electrical and thermal tortuosity of each sample are almost identical. This is because the underlying transport physics of molecular diffusion, electrical conduction and heat transfer at steady state are described by similar constitutive relations, namely Fick's, Ohm's and Fourier's laws. Diffusivity and conductivity are directly related to each other through Nernst-Einstein equation as well (Lu, 1997). Numerical simulations of these three transport processes are governed by Laplace equation with exactly the same mathematical expression, and the boundary conditions including the external driving force, outside surface and pore-solid interface are identical as well. Recognizing the consistency among diffusional, electrical and thermal tortuosities, a general definition of physical tortuosity $\tau$ can be expressed as (Barrande et al., 2007):

$$
\left(\frac{J}{\nabla F_{\text {drive }}}\right)_{\text {eff }}=\frac{\phi}{\tau^{2}}\left(\frac{J}{\nabla F_{\text {drive }}}\right)_{0}
$$

where $J$ denotes the transport flux, $\nabla F_{\text {drive }}$ denotes the driving force gradient, and the subscripts 'eff' and ' 0 ' denote the effective conductivity/diffusivity in porous media and the bulk conductivity/diffusivity in homogeneous media, respectively.

However, the general definition in Eq. (46) is not applicable to hydraulic tortuosity. As shown in Table 5 and Figure 14, the hydraulic tortuosity differs significantly from the other three physical tortuosities, especially for the porous media samples with lower porosity. Fluid permeation inside porous media not only contains the bulk movement of flow volume driven by pressure gradient, but also involves the momentum transfer from the pore wall towards the central axis of flow, so the underlying physics of hydrodynamic flow is greatly different from that of molecular diffusion, electrical conduction or heat transfer. Essentially, pore-scale fluid flow is governed by Navier-Stokes equations, which are fundamentally different from the Laplace equation that governs the other three transport phenomena. Further, due to the viscous resistance between the pore wall and fluid, the flow velocity reduces to zero at pore walls (no-slip boundary condition) and gradually increases towards the medial axes of pore channels, forming a velocity distribution as shown in Figure 17a. For the transport processes of molecular diffusion, electrical conduction and heat transfer, the flow intensity suddenly declines to zero at the pore walls, forming a uniform intensity distribution as shown in Figure 17b. Therefore, the pressure-driven fluid flow is much more hindered by the small pores inside porous media, which can make the bulk moving streamlines of fluid permeation significantly different from the transfer paths of the other three transport phenomena.

Another useful observation from Table 5 and Figure 14 is that the random walk simulation can provide tortuosity results close to the diffusional tortuosity values obtained from molecular diffusion simulations. However, the results from random walk simulation rely heavily on the numbers of random 


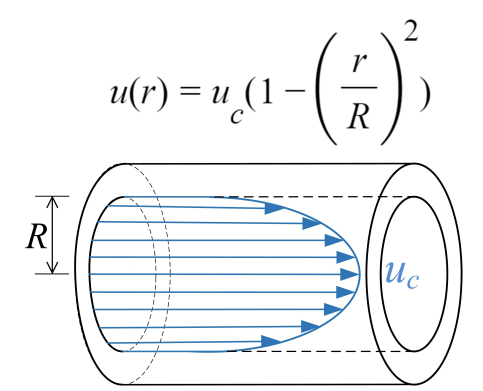

(a)

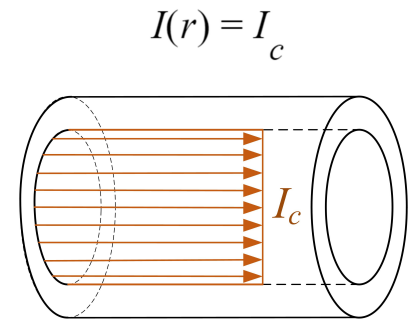

(b)

Figure 17: (a) The velocity profile of Newtonian fluid flow inside a pipe with 'no-slip' boundary condition; (b) The intensity profile of molecular diffusion, electrical conduction and heat transfer inside a pipe.

walkers as well as the simulation time step, especially for porous media with complicated microstructures. The difference between molecular diffusion and random walk simulation increases with porosity. This is because the pore space is not fully experienced by the random walkers, leading to the overestimation of tortuosity. The accuracy of random walk simulation can be improved by increasing the numbers of random walkers and time steps.

It should be noted that four different numerical methods (i.e. LBM, RWM, FDM and FVM) are used here to simulate various transport phenomena at the pore scale, so numerical errors on the computations of physical tortuosities can be different. For example, all numerical simulations directly use image voxels as the basic computing elements, but the connectivity between adjacent pore voxels follow different criteria during the discretization of governing equations. Basically, the 18-neighborconnectivity criterion is used in the LBM simulation because of the adoption of the D3Q19 lattice arrangement, while the RWM, FDM and FVM simulations follow the 6-neighbor-connectivity criterion which only permits direct exchange of dependent variables occurring between adjacent pore voxels with common faces. However, according to the comparison results in previous studies (Iwai et al., 2010; Cooper et al., 2013; Tariq et al., 2014; Cooper et al., 2016; Tjaden et al., 2017), as well as our computational experiences (as shown in Figure 14, 27 and 30), numerical errors are very tiny and negligible for the high-fidelity simulations performed on 3D high-resolution images of porous media samples. Therefore, the above analysis results on physical tortuosities are reliable, although different numerical simulation methods are used for this comparison study. Besides, image resolution and image size are important influencing factors for physical tortuosity calculation as well, which will be further discussed in $\S 6$.

\subsection{Performances of theoretical/phenomenological models}

It is evident from Table 5 and Figure 14 that there are close inverse correlations between porosity and tortuosity results computed from different image analysis methods or physical simulations. In this subsection, five different porosity-tortuosity models are applied to estimate the tortuosity results of the set of Fontainebleau sandstone samples, as expressed in Eqs. (1) to (5). As discussed in $\S 1.2$, these porosity-tortuosity relations usually contain empirical parameters or uncertain coefficients that are difficult to accurately determine, and irrational values of them may lead to great errors in tortuosity estimation. Recommended values of these adjustable parameters are found through a literature survey, and they are adopted here to assess the performances of theoretical/phenomenological models on tortuosity evaluation.

For Bruggeman relation or Archie's law (as expressed in Eq. (1)), its empirical exponent $\alpha$ usually ranges from 1.3 to 2.5 (Moctezuma-Berthier et al., 2002), and there are various methods to determine $\alpha$ for a particular porous medium (Ghanbarian et al., 2013). However, reliable values of $\alpha$ are often determined from experimental measurements or numerical simulations (Tjaden et al., 2018). The value of $\alpha$ is assigned to be 1.5 in the standard form of Bruggeman relation to estimate diffusional tortuosity 
of porous media (Tjaden et al., 2018). Besides, Mota et al. (2001) found $\alpha$ was equal to 1.8 for granular beds of spherical particles, from which the estimated hydraulic tortuosity well agreed with the experimental data. As to Comiti's model (Eq. (2)), its empirical constant $p$ varies greatly for plates with different height-side ratios (Comiti and Renaud, 1989). Mauret and Renaud (1997) found $p=0.49$ was appropriate for a capillary model of granular and fibrous beds to estimate hydraulic tortuosity, which was later confirmed by an experimental study. For the empirical model (Eq. (3)) proposed by Iversen and Jørgensen (1993), its coefficient $q=2$ was found to be appropriate for diffusional tortuosity of sandy sediments through diffusion experiments. Considering the non-spherical grains, the sphericity factor $\xi$ involved in Lanfrey's model (Eq. (5)) should be smaller than 1 (Lanfrey et al., 2010) to estimate geometrical tortuosity for natural porous media. Here, $\alpha=1.5,1.8$ and 2.5 (the upper limit), $p=0.49$, $q=2$ and $\xi=0.9$ are adopted for the above porosity-tortuosity relationships to evaluate the tortuosity values of the set of Fontainebleau sandstone samples, and the results are recorded in Table 5 and plotted

\section{Figure 14.}

In general, none of these five models can provide tortuosity values that closely match the results computed from any image analysis methods or physical simulations, which is in accordance with the remarks on theoretical/phenomenological models in $\S 1.2$. Comiti's model, Iversen's model and Du Plessis's model tend to underestimate physical tortuosities (compared with the physical simulation results), especially for the porous media samples with low porosity, while Lanfrey's model greatly overestimates the geometrical tortuosity values of the set of Fontainebleau sandstones. The primary reason of such model failures is that both model constructions and corresponding parameter calibrations are based on porous media with high porosity (such as sandy sediments, granular beds and fibrous beds). Therefore, it is no wonder that the above models can rarely provide reliable tortuosity estimations for natural porous rocks with relatively low porosity. As shown in Figure 18, a large group of pore media samples with a wide range of porosity are used to test these porosity-tortuosity models, and the results further confirm the above viewpoint.

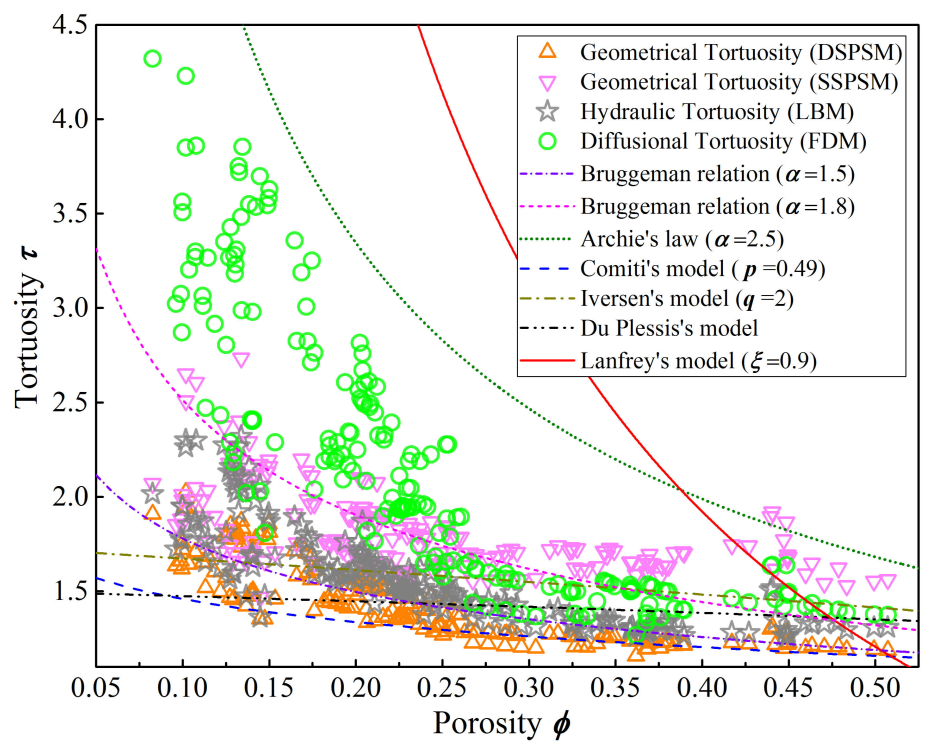

Figure 18: Tortuosity results calculated from different image analysis methods, physical simulations and porosity-tortuosity models for a group of porous media samples with a broad range of porosity (detailed information about the porous media samples are given in $\S 5$ ).

As can be seen in Table 5, Figure 14 and 18, three different values are assigned to the exponent $\alpha$ in Bruggeman relation or Archie's law to estimate tortuosity values of Fontainebleau sandstones. It seems that Bruggeman relation or Archie's law that treats tortuosity as an exponential function of tortuosity is more reasonable than the other four models, and it is possible to accurately evaluate physical tortuosities by selecting an appropriate value for the Bruggeman exponent $\alpha$. To better correlate Bruggeman model 
with tortuosity, an additional scaling factor $\beta$ was introduced by researchers, and Bruggeman model was extended to be $\tau=\beta \phi^{\alpha}$ (Tjaden et al., 2018). The crucial point is how to determine the values of adjustable parameters $\alpha$ and $\beta$ that directly affect the estimation accuracy of tortuosity. It has been proved that both $\alpha$ and $\beta$ depend on the analyzed pore microstructures, and they vary significantly even for a small class of porous media, which leads to conflicting results on the validity of Bruggeman relation in a broad range of studies (Ghanbarian et al., 2013; Tjaden et al., 2018). Therefore, the tortuosity predictions from Bruggeman relation are not always consistent with experimental data or numerical simulation results, and researchers usually alter the adjustable parameters to fit the experimental or numerical values.

It is understandable that different porous media with the same porosity may have different transport path lengths, so tortuosity cannot be a function of porosity only, especially for low-porosity rocks that possess extremely complicated microstructures with a large number of isolated and dead-end pores. Natural porous rocks are usually very different from and much more complex than the spherical structures that closely follow the initial hypotheses of porosity-tortuosity relationship derivation. The aforementioned porosity-tortuosity models do not take account of other microstructural characteristics that have considerable effects on tortuosity evaluation, such as pore size, shape, surface, distribution and connectivity. In summary, it can be stated that the porosity-tortuosity models are appropriate and reliable for porous media that are similar to the pore microstructures used to construct corresponding relationships and calibrate adjustable coefficients, but they are less suitable for complicated pore networks without adequate characterization.

\section{Relationships between geometrical and physical tortuosities}

It is usually compute-intensive to evaluate physical tortuosities via pore-scale simulations of transport processes, but geometrical tortuosity can be obtained much cheaply from image analysis. Therefore, geometrical tortuosity is commonly used as the substitute for physical tortuosities to model and predict transport properties. However, due to conceptual differences, great discrepancies exist between geometrical and physical tortuosities. As shown in Figure 14, none of the image-based methods is able to provide a geometrical tortuosity result that is close to any one of the physical tortuosities. In order to bridge such gaps, we established quantitative relationships between geometrical and physical tortuosities in this section, so that tortuosity can be better used in porous media research.

As the porosity varies, a strong correlation exists between geometrical and physical tortuosities, which can be observed in Figure 14. To confirm this trend, a large set of porous media (mainly geological materials) with diverse morphologies are used to explore the relationships between geometrical and physical tortuosities, including sandstones, sand packs, carbonate rocks (limestone), synthetic silica, bead packs, and others. The 3D micro-CT images of these porous media samples are collected from several publicly shared data sources, and due to page limit only a small subset are shown in Figure 19 in 2D form. The raw micro-CT images are in gray-scale to prepare for image analysis and physical simulation. First they are processed and segmented into binary forms by using ImageJ (ImageJ, 2016), a popular image processing tool in the digital rock physics community. As illustrated in Figure 20, 3D micro-CT images of four representative porous media samples are segmented using Otsu's method (Otsu, 1979). 


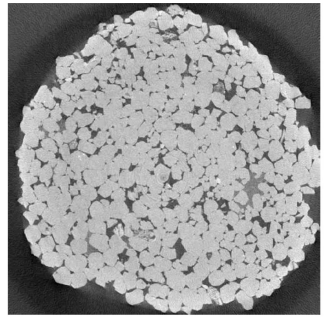

(a) Sandstone 1

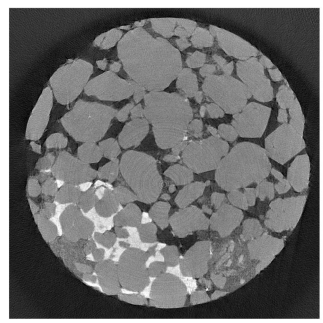

(f) Sandstone 6

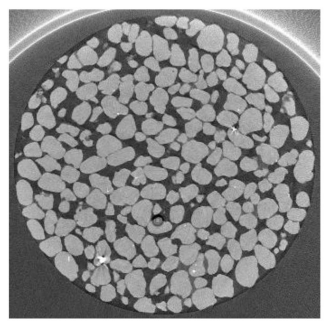

(k) Sand pack F42A

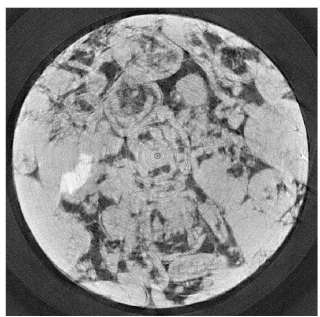

(p) Carbonate rock 1

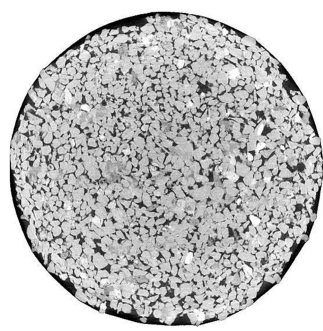

(u) Castlegate sandstone

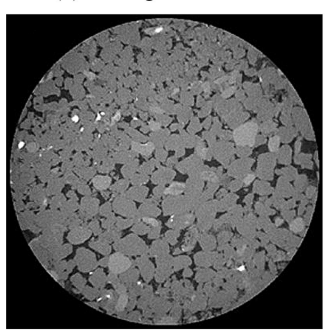

(z) Rolteigend sandstone

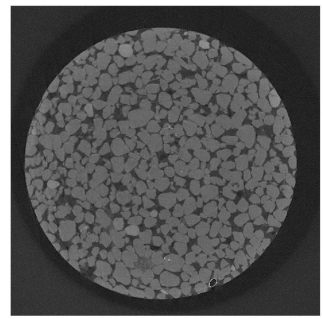

(b) Sandstone 2

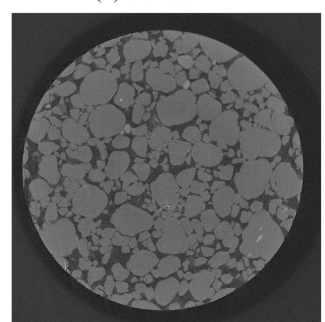

(g) Sandstone 7

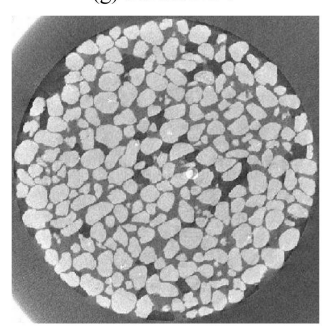

(1) Sand pack F42B



(q) Carbonate rock 2

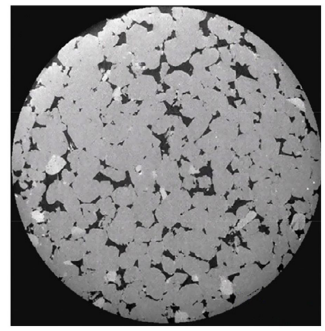

(v) Clashach sandstone

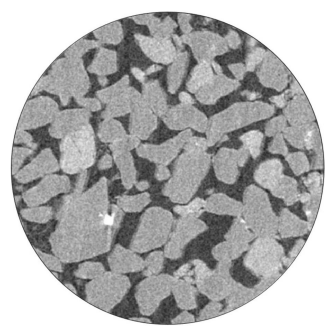

(aa) Mt. Simon sandstone

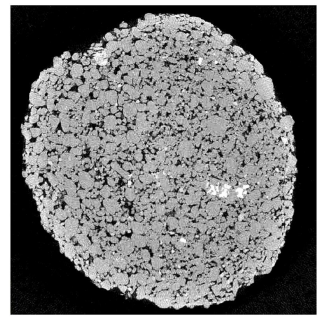

(c) Sandstone 3

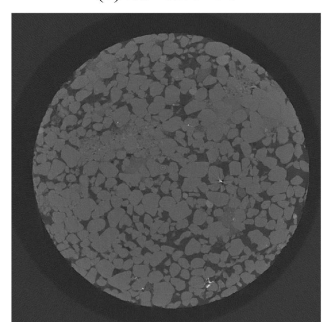

(h) Sandstone 8

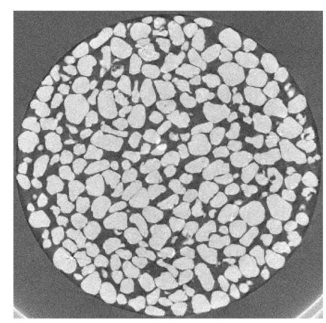

(m) Sand pack F42C



(r) Carbonate rock 3

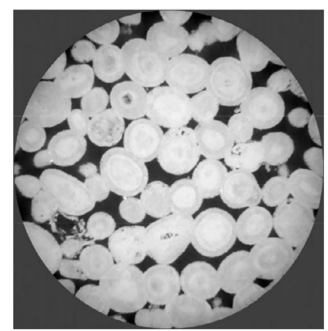

(w) Ketton limestone

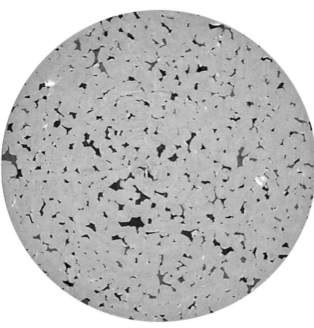

(ab) Fontainebleau sandstone

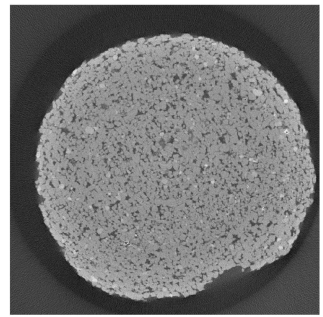

(d) Sandstone 4

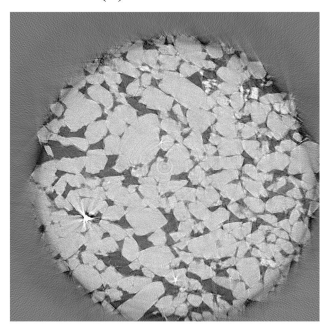

(i) Sandstone 9

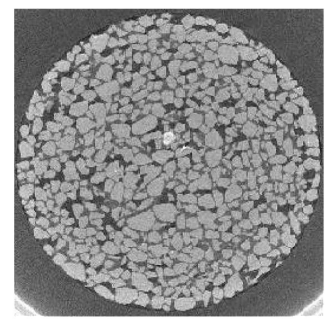

(n) Sand pack LV60A

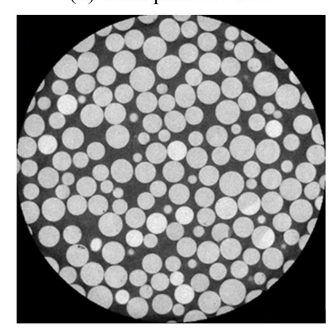

(s) Beadpack

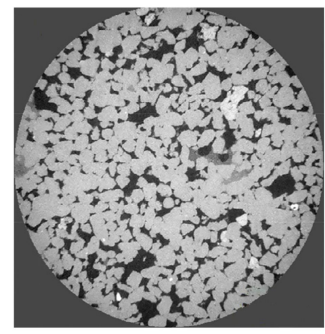

(x) Bentheimer sandstone

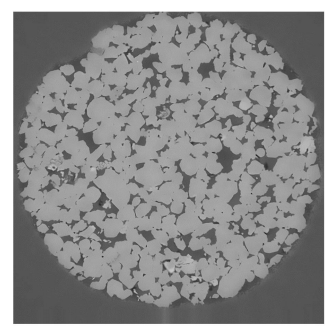

(ac) Gildehauser sandstone

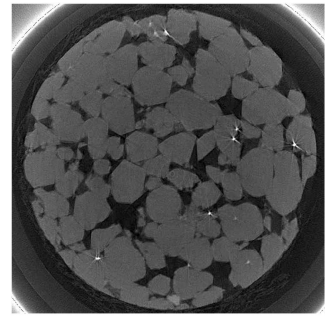

(e) Sandstone 5



(j) Berea sandstone

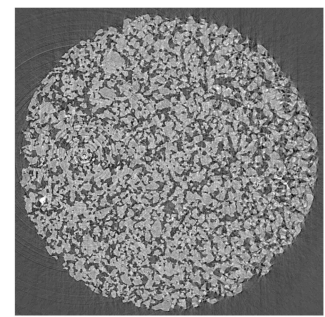

(o) Synthetic silica



(t) Mt Gambier limestone

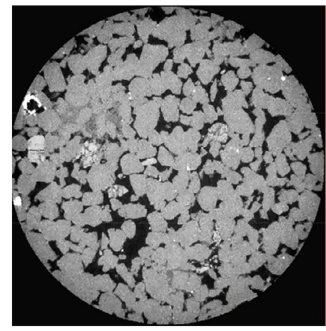

(y) Doddington sandstone

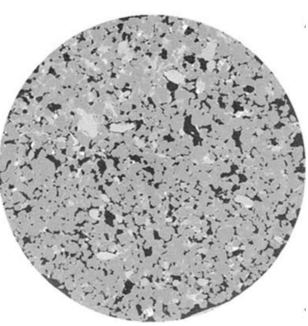

(ad) Leopard sandstone

Figure 19: The 2D cross-sections of 3D micro-CT images of different porous media samples (pore space is shown in dark): data in (a)-(r) are collected from Dong (2008); data in (s)-(u) are collected from Sheppard and Prodanovic (2015); data in (v)-(y) are collected from Shah et al. (2016); data in (z) is collected from Moon and Andrew (2019); data in (aa) is collected from Kohanpur et al. (2019); data in (ab) is collected from Latief et al. (2010); data in (ac) is collected from Berg et al. (2018); data in (ad) is collected from Herring et al. (2018). 


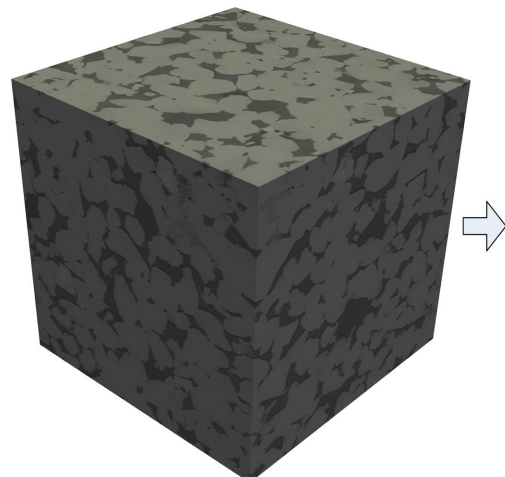

(a)

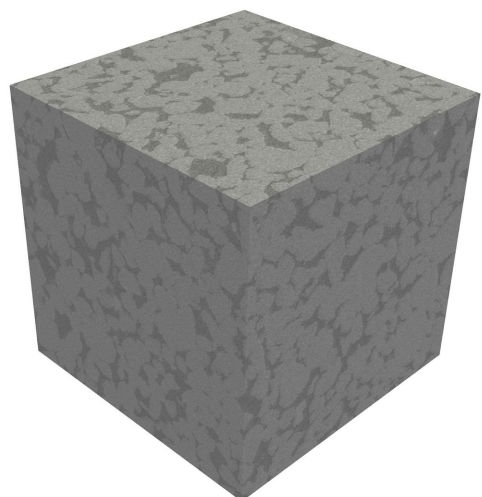

(d)

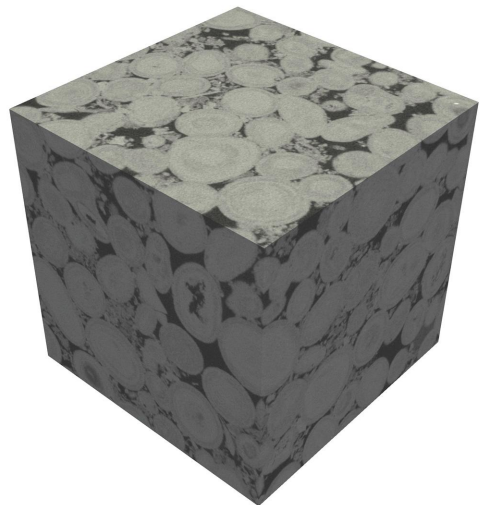

(g)

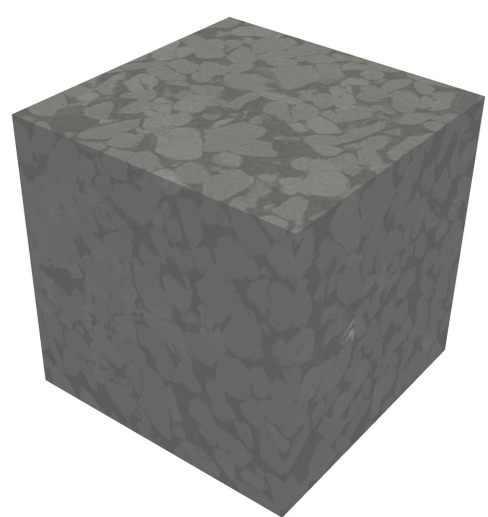

(j)



(b)

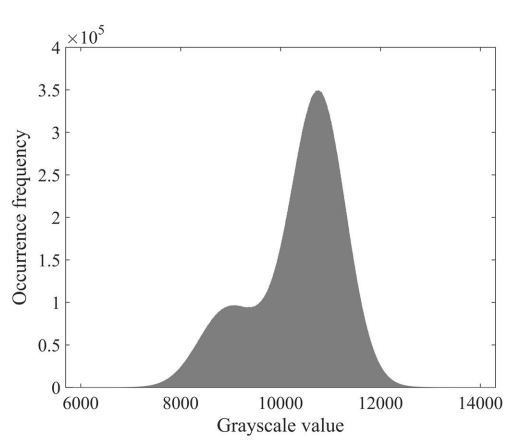

(e)

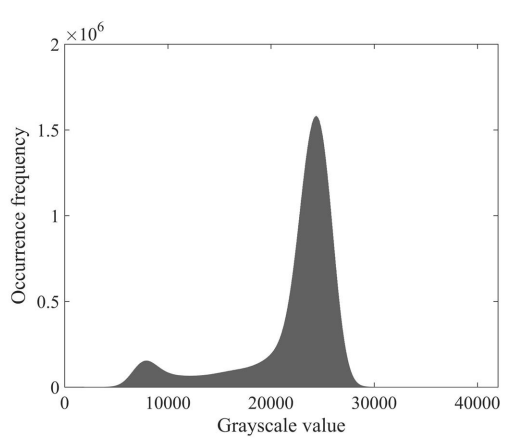

(h)

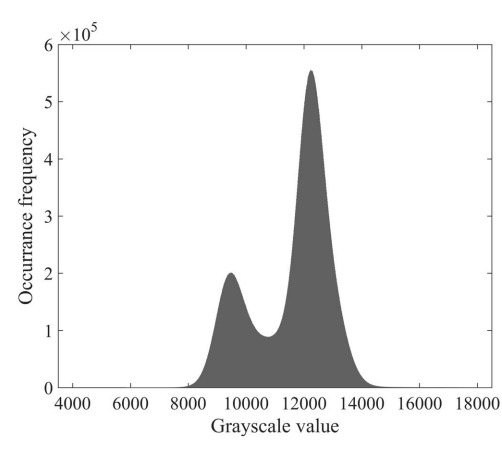

(k)

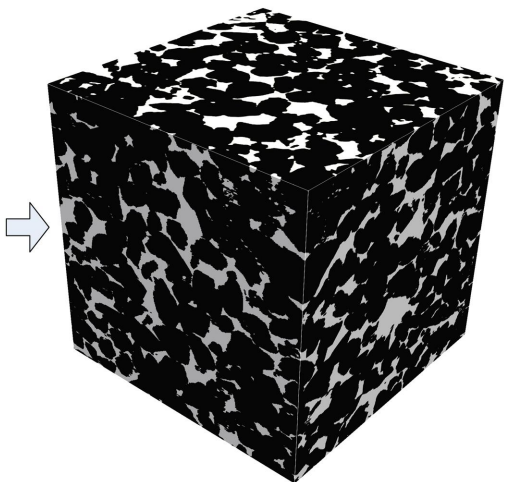

(c)

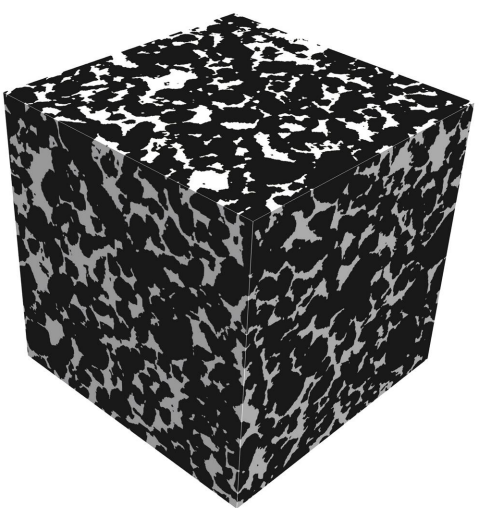

(f)

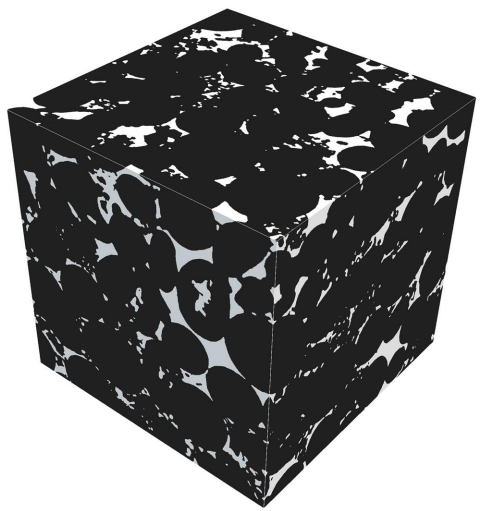

(i)

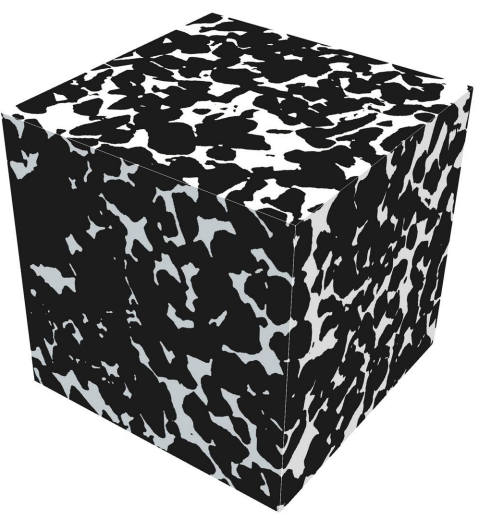

(1)

Figure 20: Segmentation of micro-CT images through Otsu's method: (a) Gildehauser sandstone, (d) Bentheimer sandstone, (c) Ketton limestone and (d) Mt. Simon sandstone; (b), (e), (h) and (k) are the histograms of grayscale values of corresponding micro-CT images; (c), (f), (i) and (l) are the segmented images in binary form (pore space is shown in white, and solid matrix is shown in black). 
The micro-CT databases employed in this work include 120 digital microstructure samples. These raw images are representative covering a diverse set of porous media, but more samples are still required in order to develop phenomenological models to link geometrical and physical tortuosities. A stochastic microstructure reconstruction method (Fu et al., 2021) has been newly developed using deep neural networks. This method is capable of quickly generating 3D digital microstructure samples by preserving the statistical equivalence, long-distance connectivity and transport properties of the training images. Here, the segmentations of the above micro-CT models are used as the guides to reconstruct new porous media samples. In total, 200 microstructure samples (120 samples from raw micro-CT images plus 80 reconstructed samples) are used in this work to investigate the relationships between geometrical and physical tortuosities, with the porosity varying from $8.61 \%$ to $50.72 \%$. Specifically, the relationship between geometrical tortuosity and hydraulic tortuosity is described in $\S 5.1$, and the relationship between geometrical tortuosity and diffusional/electrical/thermal tortuosity is described in $\S 5.2$.

\subsection{Prediction model for hydraulic tortuosity}

As shown in Figure 14, the hydraulic tortuosity lies in between the geometrical tortuosity results calculated from the DSPSM and SSPSM. This phenomenon can be easily understood by comparing the shortest pore channels with the effective flow path in Figure 2 and 4 . Thus, it is natural to use the DSPSM and the SSPSM results to develop a better approximation to hydraulic tortuosity, thereby avoiding the costly fluid flow simulations. Indeed, the same trend is also observed for the whole set of 200 porous media samples, as shown in Figure 21, where the geometrical tortuosity results calculated from the DSPSM and the SSPSM form nicely the upper and lower bounds of hydraulic tortuosity determined from LBM simulations .

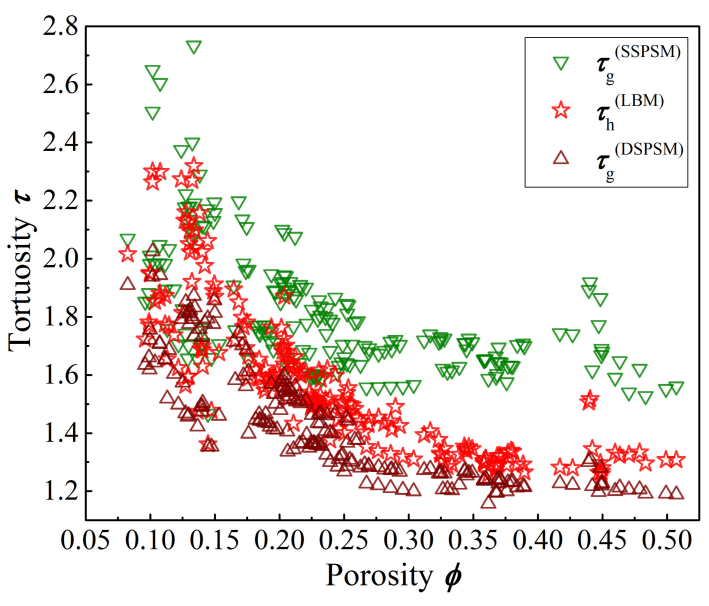

(a)

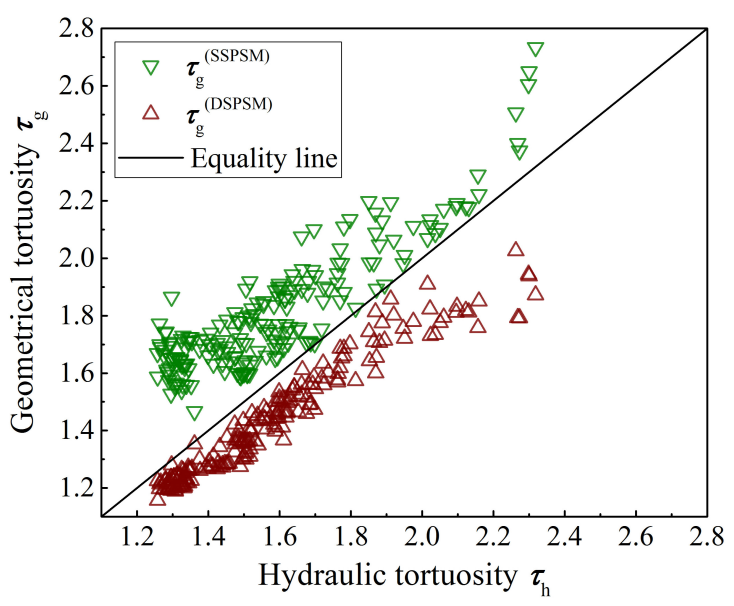

(b)

Figure 21: (a) The hydraulic tortuosity results obtained from LBM simulations and the geometrical tortuosity results obtained from the SSPSM and DSPSM for porous media samples with a wide range of porosity; (b) Comparison between the geometrical and hydraulic tortuosity results.

The above observation suggests that the average length of fluid streamlines in a porous medium is bounded by the shortest paths identified by the DSPSM and SSPSM. Based on the above data and taking into account the variation with respect to porosity, we propose the following phenomenological model to predict hydraulic tortuosity:

$$
\widetilde{\tau}_{\mathrm{h}}=a \phi^{b} \tau_{\mathrm{g}}^{(\mathrm{DSPSM})}+\left(1-a \phi^{b}\right) \tau_{\mathrm{g}}^{(\mathrm{SSPSM})}
$$

where $\widetilde{\tau}_{\mathrm{h}}$ denotes the predicted hydraulic tortuosity, $a$ and $b$ are empirical coefficients, and $\tau_{\mathrm{g}}^{(\mathrm{DSPSM})}$ and $\tau_{\mathrm{g}}^{\text {(SSPSM) }}$ are the geometrical tortuosity results calculated from the DSPSM and SSPSM, respectively. 
The set of porous media with 200 samples are randomly divided into the fitting group (70\%) and the testing group (30\%). The coefficients $a$ and $b$ are identified as 1.3100 and 0.5435 respectively, through fitting the tortuosity and porosity data extracted from the porous media samples in the fitting group. The mean error and the largest error of the fitted model are $2.99 \%$ and $7.41 \%$, respectively. The fitted model is applied to the porous media samples in the testing group, to predict the hydraulic tortuosity values based the geometrical tortuosity and porosity data. As shown in Figure 22, the predictions $\widetilde{\tau}_{\mathrm{h}}$ from the above model are in good agreement with the results $\tau_{\mathrm{h}}$ obtained from LBM simulations, and the mean error and the largest error of prediction are $3.11 \%$ and $7.19 \%$, respectively.

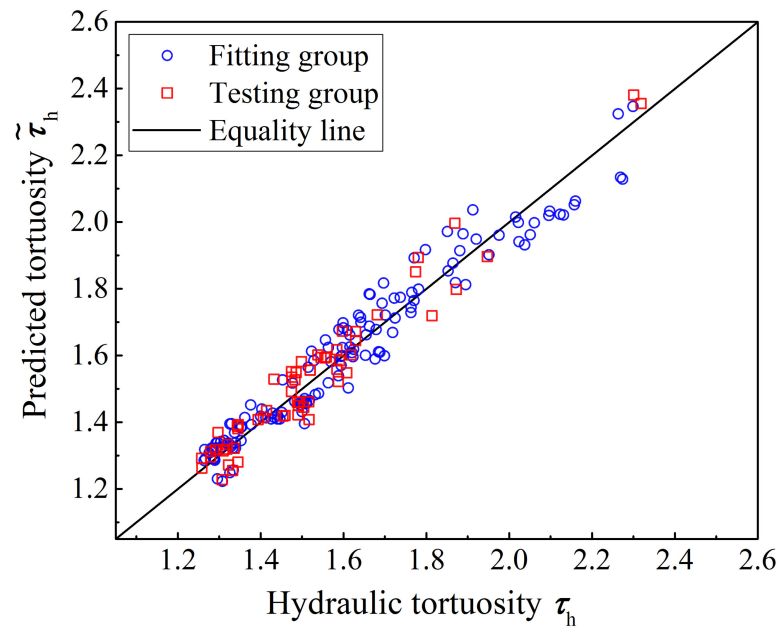

Figure 22: Comparison between the hydraulic tortuosity values $\widetilde{\tau}_{\mathrm{h}}$ predicted from the proposed model and the reference values $\tau_{\mathrm{h}}$ obtained from LBM simulations.

\subsection{Prediction model for diffusional tortuosity}

As discussed in $\S 4.2$, diffusional, electrical and thermal tortuosity are almost identical, and they are unified into a general form (as illustrated in Eq. 46). Here, we take diffusional tortuosity as the representative to establish the prediction model. The geometrical tortuosity $\tau_{\mathrm{g}}^{(\mathrm{DSPSM})}$ calculated by the DSPSM is selected for the task, because it shows a stronger correlation to diffusional tortuosity and does not involve any extra image preparation that may cause errors. As shown in Figure 14, the diffusional tortuosity $\tau_{\mathrm{d}}$ values are always larger than the geometrical tortuosity values $\tau_{\mathrm{g}}^{\text {(DSPSM) }}$, and their difference decreases with the growth of porosity $\phi$. This observation also holds for other porous media samples with wider range of porosity, as shown in Figure 23. Here, an empirical model is going to be established to link geometrical tortuosity to diffusional tortuosity. Although the gap between $\tau_{\mathrm{g}}^{(\mathrm{DSPSM})}$ and $\tau_{\mathrm{d}}$ is related to porosity, it is found that $\tau_{\mathrm{d}}$ can be accurately estimated from $\tau_{\mathrm{g}}^{(\mathrm{DSPSM})}$ without considering porosity $\phi$ through the following empirical model:

$$
\widetilde{\tau}_{\mathrm{d}}=m\left(\tau_{\mathrm{g}}^{(\mathrm{DSPSM})}\right)^{n}
$$

where $m$ and $n$ are empirical constants.

The same fitting and testing group of porous media samples are used here. The values of $m$ and $n$ are evaluated to be 0.9505 and 2.2310 respectively, though fitting the tortuosity data extracted from the porous media samples in the fitting group. The mean error and the largest error of the fitted model are $3.58 \%$ and $8.50 \%$, respectively. Applying the fitted model to the testing group, the predicted diffusional tortuosity $\widetilde{\tau}_{\mathrm{d}}$ are in good agreement with the numerical simulation result $\tau_{\mathrm{d}}$, as demonstrated in Figure 24. The mean error and the largest error of the predictions are $3.07 \%$ and $7.71 \%$, respectively. 


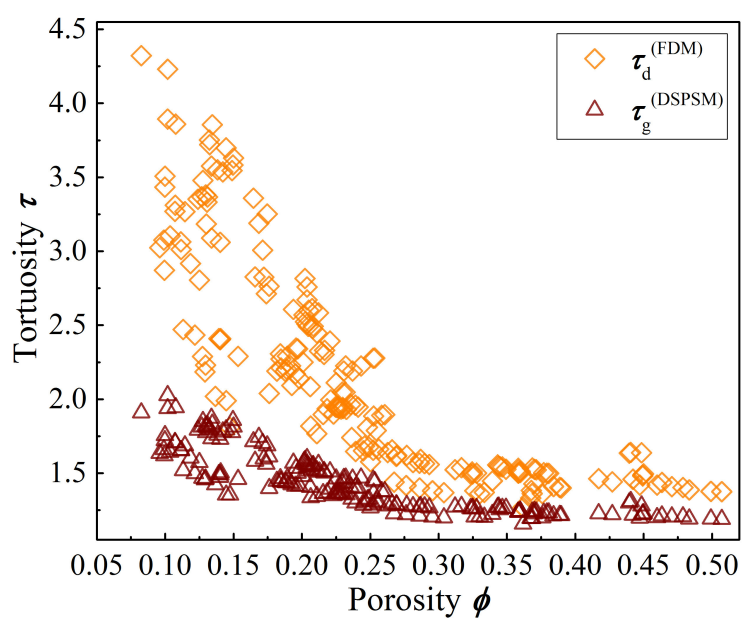

Figure 23: The diffusional tortuosity results obtained from FDM simulations and the geometrical tortuosity results obtained from the DSPSM for porous media samples with a wide range of porosity.

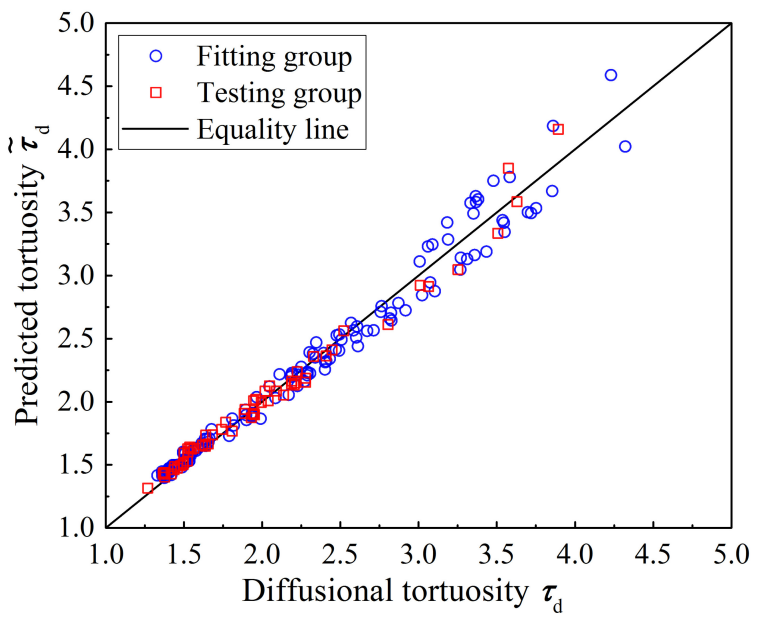

Figure 24: Comparison between the diffusional tortuosity values $\widetilde{\tau}_{\mathrm{d}}$ predicted from the proposed model and the reference values $\widetilde{\tau}_{\mathrm{d}}$ obtained from molecular diffusion simulations.

\section{Issues on REV size and resolution}

REV size and resolution (voxel size) are two critical issues that need to be carefully considered when computing tortuosity values of digital microstructures through image analysis or physical simulation. Generally, reliable pore-scale analysis requires large digital microstructure with high resolution. To serve as a good statistical representative for the specific porous medium, the REV sample needs to be sufficiently large (Costanza-Robinson et al., 2011), and to provide accurate pore space geometry close to the reality, the digital microstructure must be at a sufficiently high-resolution level (Fu et al., 2020).

Through image analysis or physical simulation, accurate tortuosity results can be obtained from large REV samples with high resolution (Borujeni et al., 2013; Peng et al., 2014; Guan et al., 2019). However, it is still a challenging task to deal with 3D REV samples with super-high resolutions, because they can contain hundreds of millions (or even billions) of voxels. High-performance computing platforms are often required to perform pore-scale analyses on these digital microstructures. In practice, the raw micro-CT images are often rescaled to a lower resolution, in order to reduce the computational cost. The resolution reduction is particularly common when using GPU platforms, as a finer REV model often exceeds the memory capacity of a single graphics card (Jones and Feng, 2016). Therefore, the 
choice of image resolution of the REV sample is often a compromise between accuracy requirement and computational complexity.

Naturally, the critical REV size and resolution for porous media depend on the characteristic length of morphological features. In this work, the correlation length is selected as the characteristic length to study the effects of image size and resolution on tortuosity evaluation, because it is related to both mean pore radius and hydraulic radius (Keehm and Mukerji, 2004). Specifically, the correlation length $l$ is defined as the distance where the two-point correlation function (Torquato, 2002) dwindles to the asymptotic value of $\phi^{2}$ (Coker et al., 1996), as illustrated in Figure 26.

\subsection{REV size}

A Fontainebleau sandstone with porosity about $14.00 \%$ is selected to study the effect of sample size on tortuosity evaluation. Its 3D micro-CT image with a resolution of $7.324 \mu \mathrm{m}$ (Hilfer and Zauner, 2011) is segmented using ImageJ (ImageJ, 2016), as shown in Figure 25. The two-point correlation function can be extracted from the binary segmentation, and the correlation length $l$ is measured as 17.50 voxels, as demonstrated in Figure 26. To study the influence of sample size on tortuosity calculation, the 3D digital microstructure is cut into cubic subsamples with side lengths $L$ of $5,10,15,20$ and 25 times of the correlation length $l$, as shown in Figure 25b.



(a)

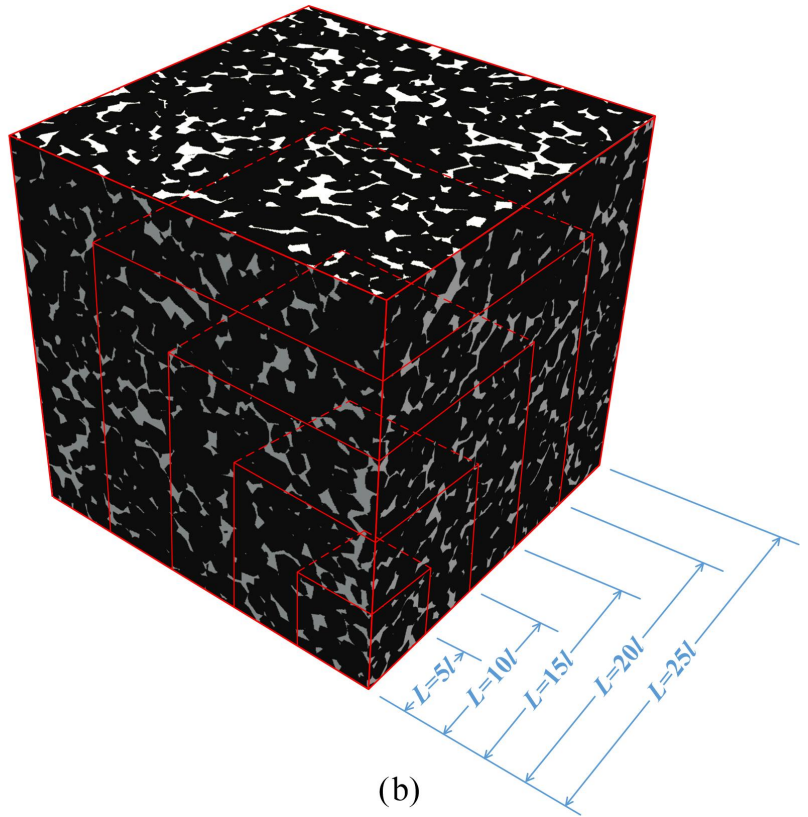

(b)

Figure 25: (a) The micro-CT image of a Fontainebleau sandstone sample with resolution of $7.324 \mu \mathrm{m}$; (b) The binary segmentation of the micro-CT image, and it is partitioned to subsamples of different sizes ( $L$ is the side length of cubic sample, and $l$ is the correlation length).

Image analysis and physical simulation are performed on these subsamples to compute geometrical and physical tortuosity, and the results are plotted in Figure 27. It can be observed that both geometrical and physical tortuosity values fluctuate greatly for small samples, especially the diffusional tortuosity computed by the RWM where the results fluctuate from 2.37 to 3.84 . As the sample size increases, both geometrical and physical tortuosities converge gradually. The larger fluctuation observed in smaller samples can be attributed to two causes: (1) the morphological characteristics of smaller samples are not statistically representative and (2) the boundary effects of various computing algorithms or numerical methods on tortuosity calculations are more visible for smaller samples. It is also observed that the tortuosity results computed from all methods become stable when the sample size $L$ increases to $20 l$, indicating the critical REV size $L_{\mathrm{c}}$ for tortuosity calculation:

$$
L_{\mathrm{c}}=20 l
$$


This critical REV size $L_{\mathrm{c}}=20 l$ can also be a valid reference for other types of porous media. But more significantly, the correlation length extracted from the two-point correlation function is demonstrated to be an effective characteristic length to determine the REV size for tortuosity evaluation.

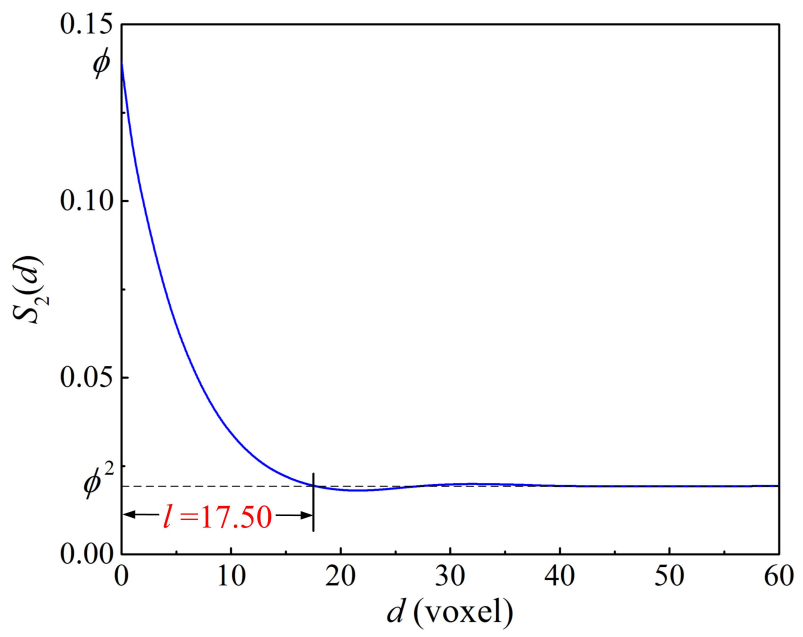

Figure 26: The two-point correlation function $S_{2}(d)$ and correlation length $l$ of the Fontainebleau sandstone sample with resolution of $7.324 \mu \mathrm{m}$.

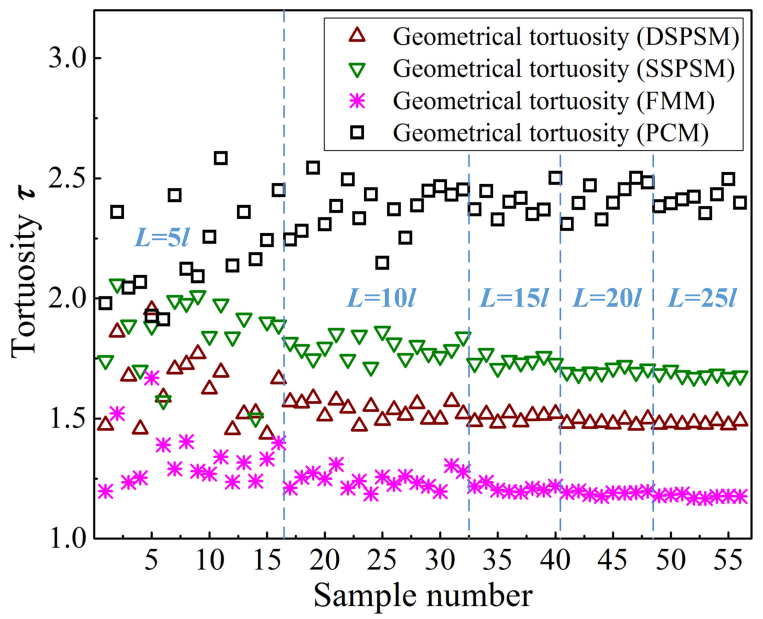

(a) Geometrical tortuosity

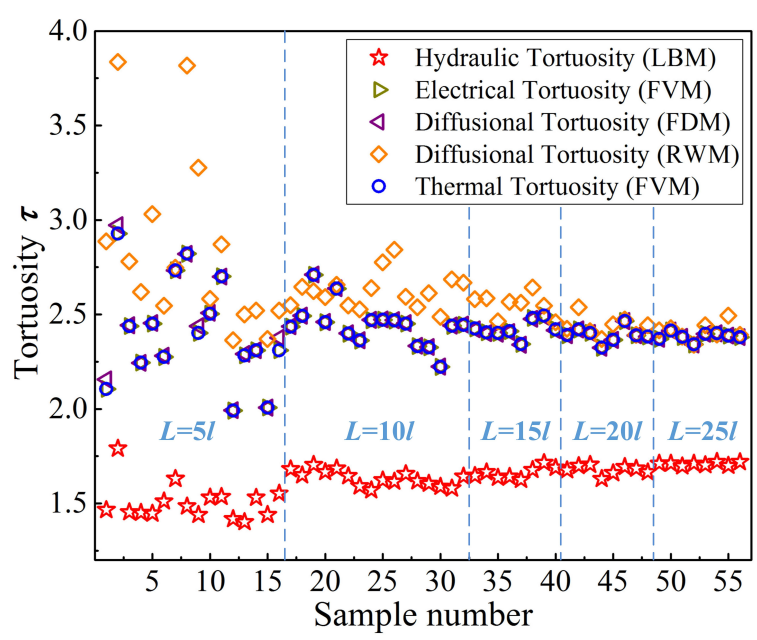

(b) Physical tortuosity

Figure 27: The effects of sample size on the calculation results of different tortuosities.

\subsection{REV resolution}

The 3D micro-CT images at four resolution levels (e.g. 3.662, 7.324, 14.648 and $29.296 \mu \mathrm{m}$ ) of the Fontainebleau sandstone (Hilfer and Zauner, 2011) are used to study the resolution effect on tortuosity evaluation, as shown in Figure 28. To avoid the sample size effect, these micro-CT images are segmented and then partitioned into subsamples with side length $L=20 l$. The correlation length $l$ of the Fontainebleau sandstone is about $128.170 \mu \mathrm{m}$, which is equal to $35.00,17.50,8.75$ and 4.38 voxles respectively for the digital microstructures from fine to coarse. The correlation length $l$ can be directly measured from the two-point correlation function curves, as illustrated in Figure 29.

Performing image analysis and pore-scale simulation on the digital microstructures with different resolutions, the geometrical and physical tortuosity values are calculated, and the results are plotted in Figure 30. The REV resolution is found to have different effects on tortuosity evaluations of the 




(a) Resolution: $3.662 \mu \mathrm{m}$

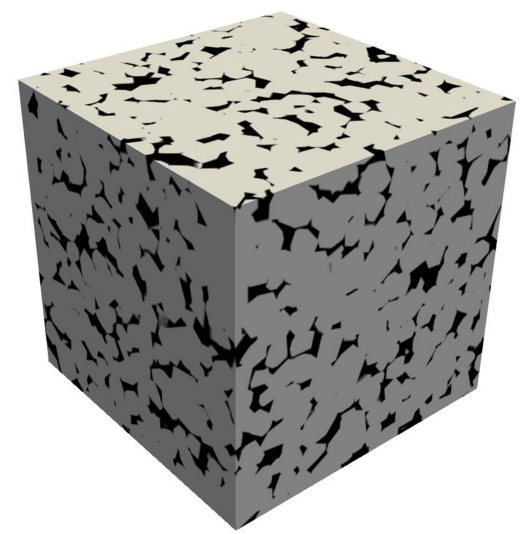

(b) Resolution: $7.324 \mu \mathrm{m}$

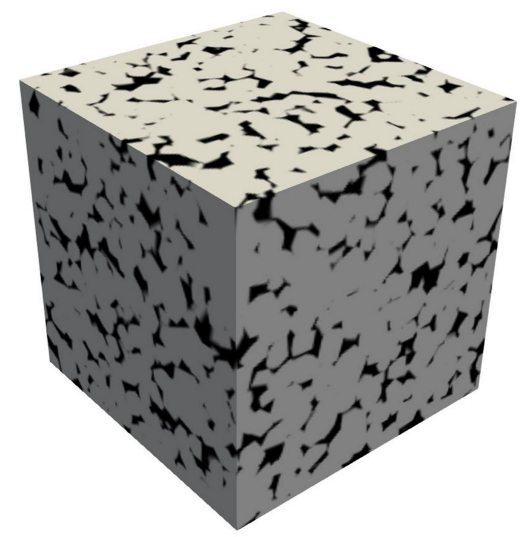

(c) Resolution: $14.648 \mu \mathrm{m}$

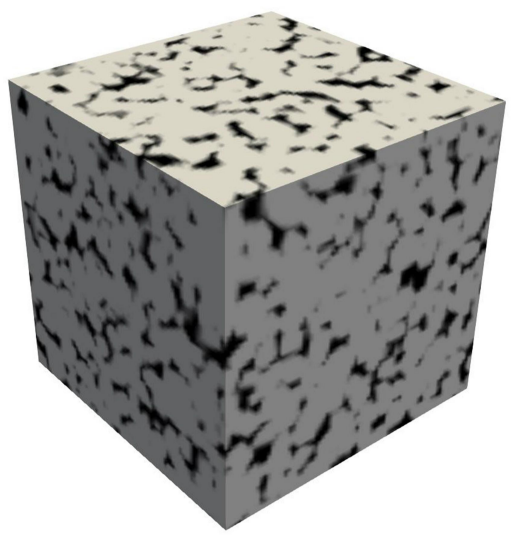

(d) Resolution: $29.296 \mu \mathrm{m}$

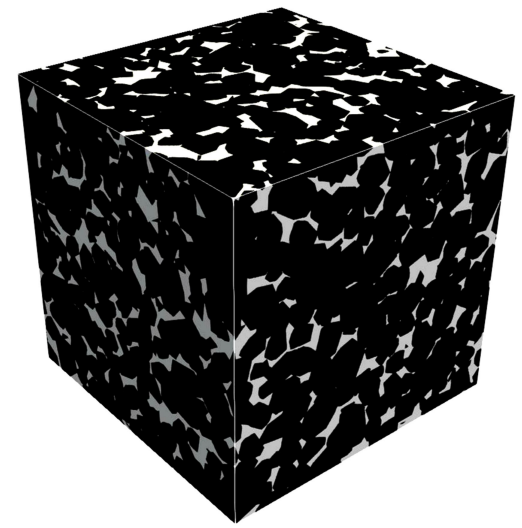

(e) Resolution: $3.662 \mu \mathrm{m}$

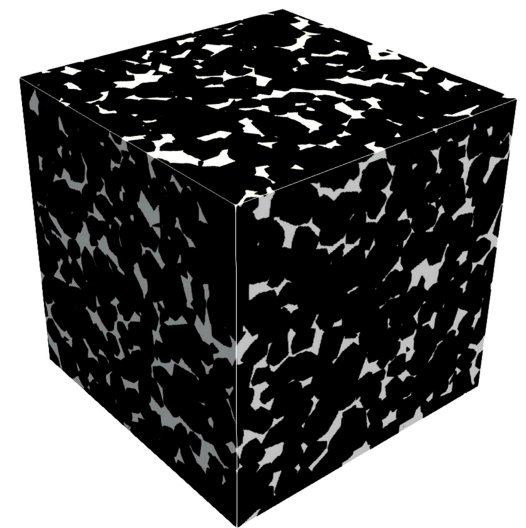

(f) Resolution: $7.324 \mu \mathrm{m}$

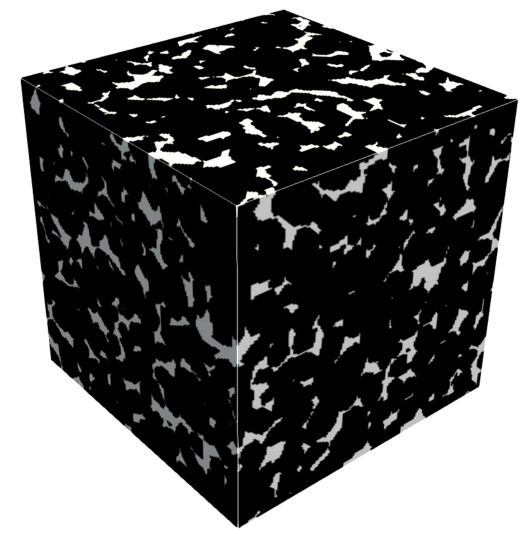

(g) Resolution: $14.648 \mu \mathrm{m}$

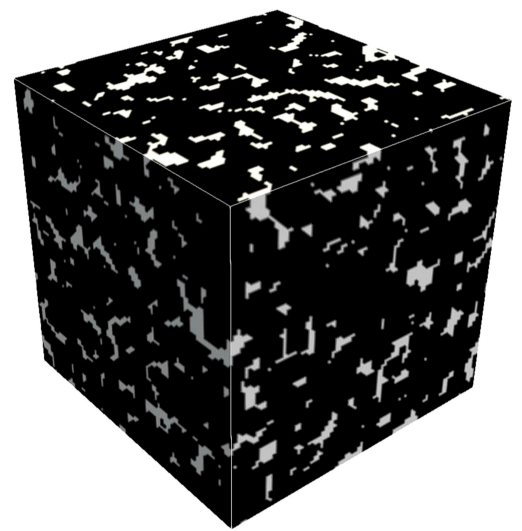

(h) Resolution: $29.296 \mu \mathrm{m}$

Figure 28: The micro-CT images and corresponding binary segmentations of a Fontainebleau sandstone sample at four different resolution level. 
four image-based methods. The geometrical tortuosity results calculated by the DSPSM and FMM are sensitive to image resolution, and they show similar increase pattern as voxel size becomes larger. There are two primary sources of error caused by the resolution effect: geometrical error and image analysis error. The difference between the acquired digital microstructure and the real microstructure is termed as the geometrical error, and this error increases as the digital microstructure becomes coarse, due to losing geometrical information during the pixel/voxel binning. Bad pore-connectivity inside the digital microstructure with low resolution usually results in a larger value of geometrical tortuosity. Image analysis error refers to the difference between the shortest pore channel identified by the algorithm and the actual shortest pore channel inside a porous microstructure. Usually, the shortest pore channel identified from a fine image is 'shorter' than that obtained from a coarse image, as illustrated in Figure 4. On the contrary, the SSPSM and PCM are less influenced by the REV resolution, and the geometrical tortuosity only slightly declines as the image resolution becomes lower. As described in $\S 2.3$, the SSPSM searches the shortest pore channel from the medial axis skeleton of the pore space, which greatly reduces the image analysis error discussed above. The PCM is very simple, and it does not consider pore-connectivity and does not involve a path searching algorithm either. As the PCM only takes into account the pore centroid position on each layer, it is usually less affected by the image resolution. Generally, the resolution effect on the four image-based methods is very much limited when the correlation length $l$ is larger than 17.5 voxels, as shown in Figure 30a. Therefore, for the calculation of geometrical tortuosity, it is recommended to use digital microstructures with the voxel size smaller than $l / 17.5$.

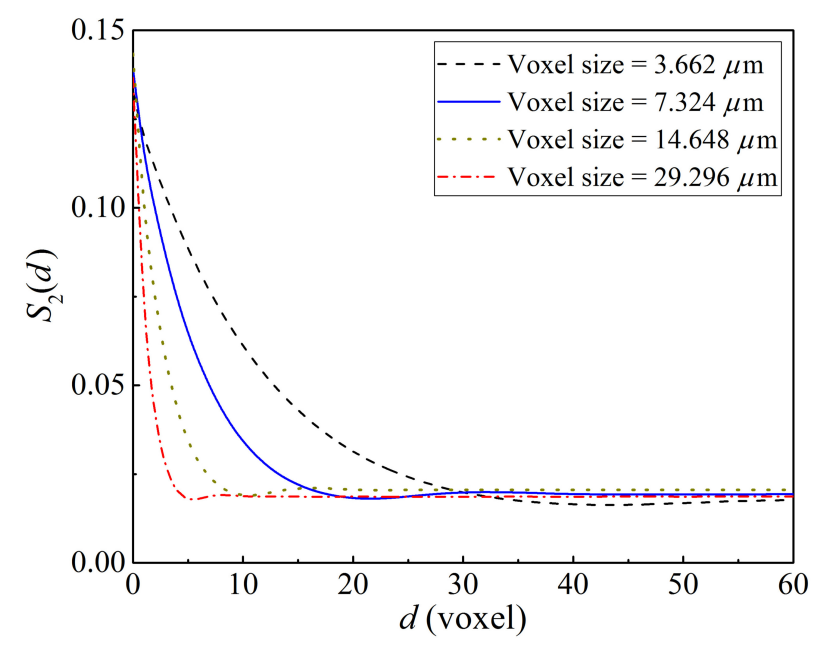

Figure 29: The two-point correlation functions $S_{2}(d)$ of digital microstructure samples at four different resolution levels.

As observed in Figure 30b, the hydraulic tortuosity evaluation is quite stable when the image resolution changes, and reasonably accurate results can be obtained on coarse images. Although hydraulic tortuosity is less affected by REV resolution, it has been proven that the resolution effect on the hydraulic conductivity (or permeability) is remarkable (Fu et al., 2020). For the evaluation of diffusional, electrical and thermal tortuosity, the resolution effect is significant. As can be seen in Figure 30b, the diffusional tortuosity results increase with the voxel size. The poor pore-connectivity inside the coarse image leads to greater retardation effects on the transport processes of molecular diffusion, electrical conduction and heat transfer, thereby overestimating the tortuosity values. Physical tortuosities are estimated from numerical simulations where image voxels are directly used as the discretization elements. Therefore, the increase of voxel size may introduce extra numerical errors. For example, when LBM is used to simulate fluid flow passing through a porous medium, the fluid-solid interface will be 'misidentified' if a suboptimal value is selected for the single relaxation time, and this error will be greatly amplified on a coarse grid (Fu et al., 2020). As shown in Figure 30b, the resolution effect on the 


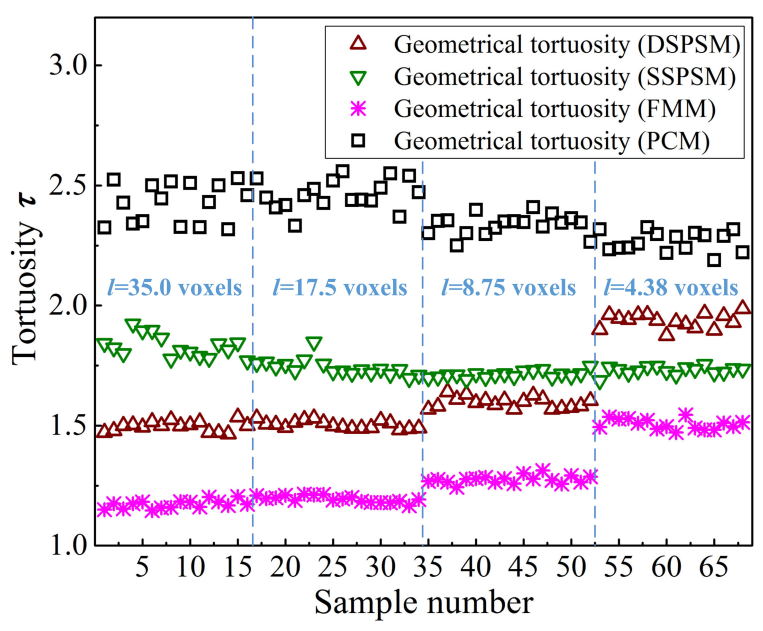

(a) Geometrical tortuosity

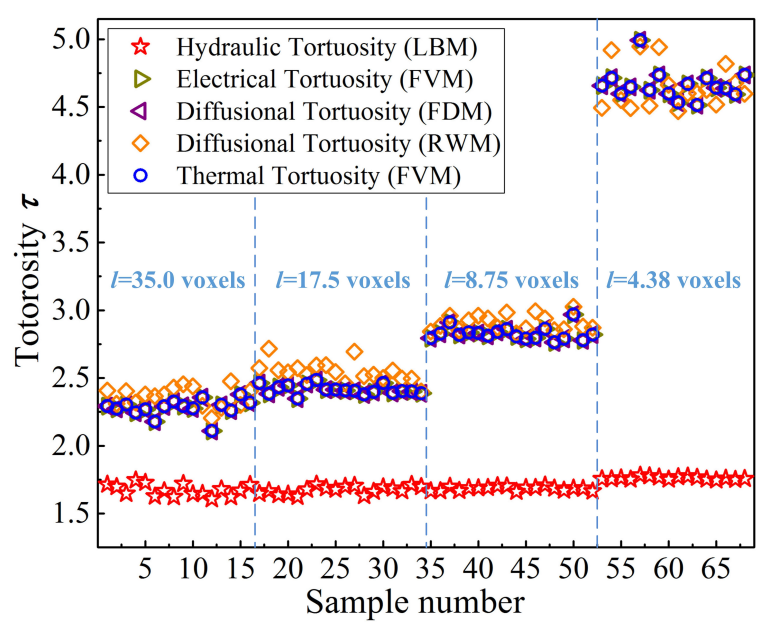

(b) Physical tortuosity

Figure 30: The effects of image resolution on calculation results of different tortuosities.

physical tortuosity calculation is generally negligible when the REV resolution is higher than $R_{\mathrm{c}}$ :

$$
R_{\mathrm{c}}=l / 17.5
$$

This critical resolution level $R_{\mathrm{c}}=l / 17.5$ can play as an effective reference for other types of porous media as well, but the key here is that the correlation length can be used as the measure to select a proper image resolution level for tortuosity evaluation.

\section{Discussion and concluding remarks}

\subsection{Discussion}

The concept of tortuosity is used to model and predict transport properties of porous media, such as permeability, effective diffusivity, electrical conductivity and thermal conductance. However, it seems that the physical tortuosities evaluated through numerical simulations of various transport phenomena are redundant, because corresponding transport properties can be directly estimated from physical simulations without the requirement of such intermediate variables. Inverse computation of physical tortuosity from transport property (as defined in Eq. (17), (23), (32) and (40)) just runs counter to the original intention of introducing tortuosity. Massive efforts have been made to directly relate tortuosity to porosity, so as to derive explicit models for tortuosity evaluation, as discussed in $\S 1.2$. The broad variety of porosity-tortuosity models usually lack general applicability for natural porous media with complicated pore networks, due to the absence of consideration of other important microstructural characteristics, which has been deeply discussed in $\S 4.3$. Besides, reliable calibrations of the empirical parameters or uncertainty coefficient involved in these porosity-tortuosity relations often require experimental measurement or numerical simulation, which heavily discounts their values in practical applications.

Geometrical tortuosity can be an effective alternative to physical tortuosities to fulfill the intended purpose in modeling and predicting transport properties, because it is a purely microstructural feature of porous media that can be quickly determined through image analysis. However, no general standard exists in extracting geometrical tortuosity from the digital microstructures of porous media, and thus uncertainties could be brought into image analysis, due to the different options in terms of image preparation, definition of the effective flow path, path searching algorithm, pixel/voxel connectivity criterion, distance metric, image resolution and other factors. Although geometrical tortuosity is conceptually sound, inconsistent results are often obtained from different image-based calculation methods, 
as discussed in $\S 4.1$. A lack of reference makes it have no way to assess the performances of different image-based methods and judge the rationality of geometrical tortuosity results. According to our own computational experience, the DSPSM and SSPSM using Dijkstra's algorithm are recommended to extract geometrical tortuosity from the digital porous media sample, because they can efficiently and stably provide geometrical tortuosity results that are the lower and upper approximations of hydraulic tortuosity respectively, as discussed in $\S 5.1$.

Tortuosity cannot simply be a characterization of pore space itself just from the geometric perspective, but it should also be related to the actual streamlines of transport flows. For electrical, diffusional and thermal flow, the transfers of charge, mass and energy depend on the available cross-sectional area of the transport path, but geometrical tortuosity doesn't take consideration of the constrictions or bottlenecks along the pore channel. As to hydrodynamic flow, the movement of fluid volume relies on both the pore size and cross-sectional shape along the permeation route, and momentum exchange is also involved into it due to the viscous effect, but these important factors are never taken into account in geometrical tortuosity. In view of this, we developed two phenomenological models based on a large group of porous media samples with a broad range of porosity in $\S 5$, aiming to bridge the gaps between geometrical and physical tortuosities. The physical tortuosity values estimated from the geometrical ones through the proposed models can be more appropriate for transport property prediction.

Along the lines of the above discussion, two issues on tortuosity may need to be further resolved: (1) If geometrical tortuosity is an intrinsic microstructural property, it should have a unique value for a particular porous medium. How to standardize the determination procedure of geometrical tortuosity to yield a unique value is the first issue. (2) Geometrical tortuosity is not exactly equivalent to physical tortuosities, so how to better understand the gaps between them is the second issue. Only after that, geometrical tortuosity together with other supplementary microstructural characteristics can completely take the role of physical tortuosities in modeling and predicting transport properties.

\subsection{Concluding remarks}

From concepts to evaluation methods, this study systematically investigates the tortuosity of porous media. The study focuses on modern approaches to evaluate the tortuosity of porous media based on digital microstructures, covering a wide range of image analysis and numerical simulation methods. The various types of tortuosity are broadly categorized into two groups: geometrical and physical tortuosity, depending on whether the analysis object is the pore space itself or the transport flow through the pore space. Physical tortuosity is further classified into four types: hydraulic, electrical, diffusional, and thermal tortuosity, corresponding to the specific transport process.

Different physical tortuosities are often used interchangeably in the literature, ignoring their distinct differences in definition and evaluation method. To clarify the situation, hydraulic, electrical, diffusional, and thermal tortuosities are examined by using numerical simulations of the corresponding transport processes in porous media. It is found that electrical, diffusional and thermal tortuosity are similar in value, but they visibly differ from hydraulic tortuosity. This is because the underlying physics of molecular diffusion, electrical conduction, and heat transfer are equivalent, and the steady-states of these transport phenomena are described by Laplace's equation. This is distinctly different from Navier-Stokes equations that govern the hydrodynamic fluid flow in porous media. Therefore, electrical, diffusional, and thermal tortuosity can be unified to be one retarding factor, but it cannot be interchangeably used with hydraulic tortuosity.

The increasing availability of high-quality digital microstructures makes it possible to perform highfidelity numerical simulations for transport flows in porous media. However, the computational cost of pore-scale simulation is high and it increases rapidly with the size of digital REVs. In contrast, geometrical tortuosity can be cheaply evaluated from image analysis, making it desirable to explore an alternative approach to evaluating physical tortuosities without involving costly physical simulation. It is found that the commonly-used image analysis methods do not provide consistent geometrical tortuosity results and they all differ greatly from the physical tortuosity values. To overcome this problem, two phenomenological models are proposed to evaluate physical tortuosities using the geometrical tortuosity, 
one for the hydraulic tortuosity prediction, and the other for the diffusional/electrical/thermal tortuosity prediction. Independent checks are performed to confirm the accuracy of these predictions.

Finally, the effects of image size and resolution on the tortuosity evaluation are analyzed as well. The correlation length extracted from two-point correlation function is found to be an effective measure to determine the critical thresholds for REV size and resolution.

\section{Acknowledgements}

The authors would like to thank the supports from China Scholarship Council (CSC No.: 201608440279), Swansea University (Zienkiewicz Scholarship), Great Britain China Center (Chinese Students Award) and the Royal Society (Ref.: IEC $\backslash$ NSFC $\backslash 191628$ ). We also greatly appreciate Imperial College London, University of Stuttgart, University College London, and Digital Rocks Portal for their publicly shared micro-CT data of various porous rocks.

\section{References}

Adler, P. M. (1992). Porous media: geometry and transports. Boston : Butterworth-Heinemann.

Adler, P. M., Jacquin, C. G., and Quiblier, J. (1990). Flow in simulated porous media. International Journal of Multiphase Flow, 16(4):691-712.

Ahmadi, M. M., Mohammadi, S., and Hayati, A. N. (2011). Analytical derivation of tortuosity and permeability of monosized spheres: A volume averaging approach. Physical Review E, 83(2):026312.

Al-Raoush, R. I. and Madhoun, I. T. (2017). Tort3d: A matlab code to compute geometric tortuosity from 3d images of unconsolidated porous media. Powder Technology, 320:99-107.

Anovitz, L. M. and Cole, D. R. (2015). Characterization and analysis of porosity and pore structures. Reviews in Mineralogy and Geochemistry, 80(1):61-164.

Archie, G. E. et al. (1942). The electrical resistivity $\log$ as an aid in determining some reservoir characteristics. Transactions of the AIME, 146(01):54-62.

Armstrong, R. T., McClure, J. E., Robins, V., Liu, Z., Arns, C. H., Schlüter, S., and Berg, S. (2019). Porous media characterization using minkowski functionals: theories, applications and future directions. Transport in Porous Media, 130(1):305-335.

Avizo (2017). ThermoFisher Scientific. Oregon, USA. Website. https ://www.thermofisher.com.

Axelsson, M. and Svensson, S. (2010). 3d pore structure characterisation of paper. Pattern Analysis and Applications, 13(2):159-172.

Backeberg, N. R., Iacoviello, F., Rittner, M., Mitchell, T. M., Jones, A. P., Day, R., Wheeler, J., Shearing, P. R., Vermeesch, P., and Striolo, A. (2017). Quantifying the anisotropy and tortuosity of permeable pathways in clay-rich mudstones using models based on x-ray tomography. Scientific Reports, 7(1):14838.

Bae, C.-J., Erdonmez, C. K., Halloran, J. W., and Chiang, Y.-M. (2013). Design of battery electrodes with dual-scale porosity to minimize tortuosity and maximize performance. Advanced Materials, $25(9): 1254-1258$.

Bærentzen, J. A. (2001). On the implementation of fast marching methods for 3d lattices.

Barrande, M., Bouchet, R., and Denoyel, R. (2007). Tortuosity of porous particles. Analytical Chemistry, 79(23):9115-9121. 
Bear, J. and Bachmat, Y. (2012). Introduction to modeling of transport phenomena in porous media, volume 4. Springer Science \& Business Media.

Berg, C. F. (2014). Permeability description by characteristic length, tortuosity, constriction and porosity. Transport in Porous Media, 103(3):381-400.

Berg, C. F. (2016). Fontainebleau 3d models. http://www.digitalrocksportal.org/projects/57.

Berg, S., Armstrong, R., and Wiegmann, A. (2018). Gildehauser sandstone. http://www. digitalrocksportal.org/projects/134.

Bini, F., Pica, A., Marinozzi, A., and Marinozzi, F. (2019). A 3d model of the effect of tortuosity and constrictivity on the diffusion in mineralized collagen fibril. Scientific Reports, 9(1):2658.

Bird, R. B. (2002). Transport phenomena. Applied Mechanics Reviews, 55(1):R1-R4.

Blunt, M. J., Bijeljic, B., Dong, H., Gharbi, O., Iglauer, S., Mostaghimi, P., Paluszny, A., and Pentland, C. (2013). Pore-scale imaging and modelling. Advances in Water Resources, 51:197-216.

Bodla, K. K., Murthy, J. Y., and Garimella, S. V. (2010). Microtomography-based simulation of transport through open-cell metal foams. Numerical Heat Transfer, Part A: Applications, 58(7):527544.

Borujeni, A. T., Lane, N., Thompson, K., and Tyagi, M. (2013). Effects of image resolution and numerical resolution on computed permeability of consolidated packing using $\mathrm{lb}$ and fem pore-scale simulations. Computers \& Fluids, 88:753-763.

Botchwey, E. A., Dupree, M. A., Pollack, S. R., Levine, E. M., and Laurencin, C. T. (2003). Tissue engineered bone: Measurement of nutrient transport in three-dimensional matrices. Journal of Biomedical Materials Research Part A: An Official Journal of The Society for Biomaterials, The Japanese Society for Biomaterials, and The Australian Society for Biomaterials and the Korean Society for Biomaterials, 67(1):357-367.

Boudreau, B. P. (1996). The diffusive tortuosity of fine-grained unlithified sediments. Geochimica et Cosmochimica Acta, 60(16):3139-3142.

Brodkey, R. S. and Hershey, H. C. (2003). Transport phenomena: a unified aprroach. Brodkey publishing.

Brown, L., Abdulaziz, R., Tjaden, B., Inman, D., Brett, D., and Shearing, P. (2016). Investigating microstructural evolution during the electroreduction of uo2 to $\mathrm{u}$ in licl-kcl eutectic using focused ion beam tomography. Journal of Nuclear Materials, 480:355-361.

Bruggeman, V. D. (1935). Berechnung verschiedener physikalischer konstanten von heterogenen substanzen. i. dielektrizitätskonstanten und leitfähigkeiten der mischkörper aus isotropen substanzen. Annalen Der Physik, 416(7):636-664.

Brun, E., Vicente, J., Topin, F., and Occelli, R. (2008). Imorph: A 3d morphological tool to fully analyse all kind of cellular materials. Cellular Metals for Structural and Functional Applications.

Bultreys, T., De Boever, W., and Cnudde, V. (2016). Imaging and image-based fluid transport modeling at the pore scale in geological materials: A practical introduction to the current state-of-the-art. Earth-Science Reviews, 155:93-128.

Cai, J., Wei, W., Hu, X., and Wood, D. A. (2017). Electrical conductivity models in saturated porous media: A review. Earth-Science Reviews, 171:419-433.

Carman, P. C. (1937). Fluid flow through granular beds. Transactions of the Institution of Chemical Engineers, 50:150-166. 
Carman, P. C. (1956). Flow of gases through porous media. Butterworths Scientific Publications: London.

Cecen, A., Wargo, E., Hanna, A., Turner, D., Kalidindi, S., and Kumbur, E. (2012). 3-d microstructure analysis of fuel cell materials: spatial distributions of tortuosity, void size and diffusivity. Journal of The Electrochemical Society, 159(3):B299-B307.

Chen-Wiegart, Y.-c. K., DeMike, R., Erdonmez, C., Thornton, K., Barnett, S. A., and Wang, J. (2014). Tortuosity characterization of $3 \mathrm{~d}$ microstructure at nano-scale for energy storage and conversion materials. Journal of Power Sources, 249:349-356.

Cherkassky, B. V., Goldberg, A. V., and Radzik, T. (1996). Shortest paths algorithms: Theory and experimental evaluation. Mathematical Programming, 73(2):129-174.

Chung, D.-W., Ebner, M., Ely, D. R., Wood, V., and García, R. E. (2013). Validity of the bruggeman relation for porous electrodes. Modelling and Simulation in Materials Science and Engineering, 21(7):074009.

Civan, F. (2011). Porous media transport phenomena. John Wiley \& Sons.

Clennell, M. B. (1997). Tortuosity: a guide through the maze. Geological Society, London, Special Publications, 122(1):299-344.

Coelho, D., Shapiro, M., Thovert, J., and Adler, P. M. (1996). Electroosmotic phenomena in porous media. Journal of Colloid and Interface Science, 181(1):169-190.

Coker, D. A., Torquato, S., and Dunsmuir, J. H. (1996). Morphology and physical properties of fontainebleau sandstone via a tomographic analysis. Journal of Geophysical Research: Solid Earth, 101(B8):17497-17506.

Coleman, S. and Vassilicos, J. (2008). Transport properties of saturated and unsaturated porous fractal materials. Physical Review Letters, 100(3):035504.

Comiti, J. and Renaud, M. (1989). A new model for determining mean structure parameters of fixed beds from pressure drop measurements: application to beds packed with parallelepipedal particles. Chemical Engineering Science, 44(7):1539-1545.

Cooper, S. (2015). Quantifying the transport properties of solid oxide fuel cell electrodes.

Cooper, S., Bertei, A., Shearing, P., Kilner, J., and Brandon, N. (2016). Taufactor: An open-source application for calculating tortuosity factors from tomographic data. SoftwareX, 5:203-210.

Cooper, S., Eastwood, D., Gelb, J., Damblanc, G., Brett, D., Bradley, R., Withers, P., Lee, P., Marquis, A., Brandon, N., et al. (2014). Image based modelling of microstructural heterogeneity in lifepo4 electrodes for li-ion batteries. Journal of Power Sources, 247:1033-1039.

Cooper, S. J., Kishimoto, M., Tariq, F., Bradley, R. S., Marquis, A. J., Brandon, N. P., Kilner, J. A., and Shearing, P. R. (2013). Microstructural analysis of an lscf cathode using in situ tomography and simulation. ECS Transactions, 57(1):2671-2678.

Corapcioglu, M. Y. (1996). Advances in Porous Media, volume 3. Elsevier.

Costanza-Robinson, M. S., Estabrook, B. D., and Fouhey, D. F. (2011). Representative elementary volume estimation for porosity, moisture saturation, and air-water interfacial areas in unsaturated porous media: Data quality implications. Water Resources Research, 47(7).

David, C. (1993). Geometry of flow paths for fluid transport in rocks. Journal of Geophysical Research: Solid Earth, 98(B7):12267-12278. 
De Lima, O. and Niwas, S. (2000). Estimation of hydraulic parameters of shaly sandstone aquifers from geoelectrical measurements. Journal of Hydrology, 235(1-2):12-26.

de Lima, O. A. (1995). Water saturation and permeability from resistivity, dielectric, and porosity logs. Geophysics, 60(6):1756-1764.

Degruyter, W., Burgisser, A., Bachmann, O., and Malaspinas, O. (2010). Synchrotron x-ray microtomography and lattice boltzmann simulations of gas flow through volcanic pumices. Geosphere, $6(5): 470-481$.

Desrues, J., Viggiani, G., and Besuelle, P. (2010). Advances in X-ray Tomography for Geomaterials, volume 118. John Wiley \& Sons.

Devarapalli, R. S., Islam, A., Faisal, T. F., Sassi, M., and Jouiad, M. (2017). Micro-ct and fib-sem imaging and pore structure characterization of dolomite rock at multiple scales. Arabian Journal of Geosciences, 10(16):361.

Dias, R., Teixeira, J. A., Mota, M., and Yelshin, A. (2006). Tortuosity variation in a low density binary particulate bed. Separation and Purification Technology, 51(2):180-184.

Dong, H. (2008). Micro-CT imaging and pore network extraction. PhD thesis, Department of Earth Science and Engineering, Imperial College London.

Du Plessis, J. P. and Masliyah, J. H. (1991). Flow through isotropic granular porous media. Transport in porous media, 6(3):207-221.

Duda, A., Koza, Z., and Matyka, M. (2011). Hydraulic tortuosity in arbitrary porous media flow. Physical Review E, 84(3):036319.

Epstein, N. (1989). On tortuosity and the tortuosity factor in flow and diffusion through porous media. Chemical Engineering Science, 44(3):777-779.

Ferguson, J. C., Panerai, F., Borner, A., and Mansour, N. N. (2018). Puma: the porous microstructure analysis software. SoftwareX, 7:81-87.

Finegan, D. P., Cooper, S. J., Tjaden, B., Taiwo, O. O., Gelb, J., Hinds, G., Brett, D. J., and Shearing, P. R. (2016). Characterising the structural properties of polymer separators for lithium-ion batteries in 3d using phase contrast x-ray microscopy. Journal of Power Sources, 333:184-192.

Fredrich, J., DiGiovanni, A., and Noble, D. (2006). Predicting macroscopic transport properties using microscopic image data. Journal of Geophysical Research: Solid Earth, 111(B3).

Fu, J., Cui, S., Cen, S., and Li, C. (2021). Statistical characterization and reconstruction of heterogeneous microstructures using deep neural network. Computer Methods in Applied Mechanics and Engineering, 373:113516.

Fu, J., Dong, J., Wang, Y., Ju, Y., Owen, D. R. J., and Li, C. (2020). Resolution effect: an error correction model for intrinsic permeability of porous media estimated from lattice boltzmann method. Transport in Porous Media, 132:627-656.

Gariboldi, M. I. and Best, S. M. (2015). Effect of ceramic scaffold architectural parameters on biological response. Frontiers in Bioengineering and Biotechnology, 3:151.

Garrouch, A. A., Ali, L., and Qasem, F. (2001). Using diffusion and electrical measurements to assess tortuosity of porous media. Industrial \& Engineering Chemistry Research, 40(20):4363-4369.

GeoDict (2020). Math2Market. Kaiserslautern, Germany. Website. http://www.geodict.com. 
Georgali, B. and Tsakiridis, P. (2005). Microstructure of fire-damaged concrete. a case study. Cement and Concrete Composites, 27(2):255-259.

Ghanbarian, B., Hunt, A. G., Ewing, R. P., and Sahimi, M. (2013). Tortuosity in porous media: a critical review. Soil Science Society of America Journal, 77(5):1461-1477.

Ginzbourg, I. and Adler, P. M. (1994). Boundary flow condition analysis for the three-dimensional lattice boltzmann model. Journal de Physique II, 4(2):191-214.

Gommes, C. J., Bons, A.-J., Blacher, S., Dunsmuir, J. H., and Tsou, A. H. (2009). Practical methods for measuring the tortuosity of porous materials from binary or gray-tone tomographic reconstructions. AIChE Journal, 55(8):2000-2012.

Gostovic, D., Smith, z. J., Kundinger, D., Jones, K., and Wachsman, E. (2007). Three-dimensional reconstruction of porous lscf cathodes. Electrochemical and Solid-state Letters, 10(12):B214-B217.

Grathwohl, P. (2012). Diffusion in natural porous media: contaminant transport, sorption/desorption and dissolution kinetics, volume 1. Springer Science \& Business Media.

Guan, K. M., Nazarova, M., Guo, B., Tchelepi, H., Kovscek, A. R., and Creux, P. (2019). Effects of image resolution on sandstone porosity and permeability as obtained from x-ray microscopy. Transport in Porous Media, 127(1):233-245.

Guo, Z. and Zhao, T. (2005). A lattice boltzmann model for convection heat transfer in porous media. Numerical Heat Transfer, Part B, 47(2):157-177.

Hassouna, M. S. and Farag, A. A. (2007). Multistencils fast marching methods: A highly accurate solution to the eikonal equation on cartesian domains. IEEE Transactions on Pattern Analysis and Machine Intelligence, 29(9):1563-1574.

He, W., Zou, J., Wang, B., Vilayurganapathy, S., Zhou, M., Lin, X., Zhang, K. H., Lin, J., Xu, P., and Dickerson, J. H. (2013). Gas transport in porous electrodes of solid oxide fuel cells: a review on diffusion and diffusivity measurement. Journal of Power Sources, 237:64-73.

Herring, A., Sheppard, A., Turner, M., and Beeching, L. (2018). Multiphase flows in sandstones. http://www.digitalrocksportal .org/projects/135.

Hilfer, R. (2002). Review on scale dependent characterization of the microstructure of porous media. Transport in Porous Media, 46(2-3):373-390.

Hilfer, R. and Zauner, T. (2011). High-precision synthetic computed tomography of reconstructed porous media. Physical Review E, 84(6):062301.

Hillel, D. (2003). Introduction to environmental soil physics. Elsevier.

Holzer, L., Wiedenmann, D., Münch, B., Keller, L., Prestat, M., Gasser, P., Robertson, I., and Grobéty, B. (2013). The influence of constrictivity on the effective transport properties of porous layers in electrolysis and fuel cells. Journal of Materials Science, 48(7):2934-2952.

Hosticka, B., Norris, P., Brenizer, J., and Daitch, C. (1998). Gas flow through aerogels. Journal of Non-crystalline Solids, 225:293-297.

Hu, L. B., Savidge, C., Rizzo, D. M., Hayden, N., Hagadorn, J. W., and Dewoolkar, M. (2012). Commonly used porous building materials: Geomorphic pore structure and fluid transport. Journal of Materials in Civil Engineering, 25(12):1803-1812. 
Hutzenlaub, T., Asthana, A., Becker, J., Wheeler, D., Zengerle, R., and Thiele, S. (2013). Fib/sem-based calculation of tortuosity in a porous licoo2 cathode for a li-ion battery. Electrochemistry Communications, 27:77-80.

ImageJ (2016). Website. https://imagej.net/Welcome.

Ioannidis, M., Kwiecien, M., and Chatzis, I. (1997). Electrical conductivity and percolation aspects of statistically homogeneous porous media. Transport in Porous Media, 29(1):61-83.

Iversen, N. and Jørgensen, B. B. (1993). Diffusion coefficients of sulfate and methane in marine sediments: Influence of porosity. Geochimica et Cosmochimica Acta, 57(3):571-578.

Iwai, H., Shikazono, N., Matsui, T., Teshima, H., Kishimoto, M., Kishida, R., Hayashi, D., Matsuzaki, K., Kanno, D., Saito, M., et al. (2010). Quantification of sofc anode microstructure based on dual beam fib-sem technique. Journal of Power Sources, 195(4):955-961.

Javadpour, F. et al. (2009). Nanopores and apparent permeability of gas flow in mudrocks (shales and siltstone). Journal of Canadian Petroleum Technology, 48(08):16-21.

Jin, G., Patzek, T., and Silin, D. (2004). Direct prediction of the absolute permeability of unconsolidated and consolidated reservoir rock. spe 90084. In 2003 SPE Annual Technical Conference and Exhibition (Houston, Texas, USA), SPE.

Jones, B. and Feng, Y. (2016). Effect of image scaling and segmentation in digital rock characterisation. Computational Particle Mechanics, 3(2):201-213.

Jørgensen, P. S., Ebbehøj, S. L., and Hauch, A. (2015). Triple phase boundary specific pathway analysis for quantitative characterization of solid oxide cell electrode microstructure. Journal of Power Sources, 279:686-693.

Jørgensen, P. S., Hansen, K. V., Larsen, R., and Bowen, J. R. (2011). Geometrical characterization of interconnected phase networks in three dimensions. Journal of Microscopy, 244(1):45-58.

Kaczmarek, Ł. D., Zhao, Y., Konietzky, H., Wejrzanowski, T., and Maksimczuk, M. (2017). Numerical approach in recognition of selected features of rock structure from hybrid hydrocarbon reservoir samples based on microtomography. Studia Geotechnica et Mechanica, 39(1):13-26.

Karanikola, V., Corral, A. F., Jiang, H., Sáez, A. E., Ela, W. P., and Arnold, R. G. (2017). Effects of membrane structure and operational variables on membrane distillation performance. Journal of Membrane Science, 524:87-96.

Keehm, Y. and Mukerji, T. (2004). Permeability and relative permeability from digital rocks: Issues on grid resolution and representative elementary volume. In SEG Technical Program Expanded Abstracts 2004, pages 1654-1657. Society of Exploration Geophysicists.

Kehrwald, D., Shearing, P. R., Brandon, N. P., Sinha, P. K., and Harris, S. J. (2011). Local tortuosity inhomogeneities in a lithium battery composite electrode. Journal of The Electrochemical Society, 158(12):A1393-A1399.

Keller, L. M., Hilger, A., and Manke, I. (2015). Impact of sand content on solute diffusion in opalinus clay. Applied Clay Science, 112:134-142.

Kishimoto, M., Iwai, H., Miyawaki, K., Saito, M., and Yoshida, H. (2013). Improvement of the sub-gridscale model designed for $3 \mathrm{~d}$ numerical simulation of solid oxide fuel cell electrodes using an adaptive power index. Journal of Power Sources, 223:268-276.

Kohanpur, A. H., Valocchi, A., and Crandall, D. (2019). Micro-ct images of a heterogeneous mt. simon sandstone sample. http://www.digitalrocksportal.org/projects/247. 
Kolitcheff, S., Jolimaitre, E., Hugon, A., Verstraete, J., Carrette, P.-L., and Tayakout-Fayolle, M. (2017). Tortuosity of mesoporous alumina catalyst supports: Influence of the pore network organization. Microporous and Mesoporous Materials, 248:91-98.

Koponen, A., Kataja, M., and Timonen, J. (1997). Permeability and effective porosity of porous media. Physical Review E, 56(3):3319.

Koponen, A., Kataja, M., and Timonen, J. v. (1996). Tortuous flow in porous media. Physical Review E, 54(1):406.

Kozeny, J. (1927). Über kapillare leitung des wassers im boden, vol. 136. Sitzungsber Akad Wiss, Wien.

Kreamer, D. K., Weeks, E. P., and Thompson, G. M. (1988). A field technique to measure the tortuosity and sorption-affected porosity for gaseous diffusion of materials in the unsaturated zone with experimental results from near barnwell, south carolina. Water Resources Research, 24(3):331-341.

Krüger, T., Kusumaatmaja, H., Kuzmin, A., Shardt, O., Silva, G., and Viggen, E. M. (2017). The lattice boltzmann method. Springer International Publishing, 10:978-3.

Kutay, M. E., Aydilek, A. H., and Masad, E. (2006). Laboratory validation of lattice boltzmann method for modeling pore-scale flow in granular materials. Computers and Geotechnics, 33(8):381-395.

Lai, J., Wang, G., Wang, Z., Chen, J., Pang, X., Wang, S., Zhou, Z., He, Z., Qin, Z., and Fan, X. (2018). A review on pore structure characterization in tight sandstones. Earth-Science Reviews, 177:436-457.

Landesfeind, J., Hattendorff, J., Ehrl, A., Wall, W. A., and Gasteiger, H. A. (2016). Tortuosity determination of battery electrodes and separators by impedance spectroscopy. Journal of The Electrochemical Society, 163(7):A1373-A1387.

Lanfrey, P.-Y., Kuzeljevic, Z., and Dudukovic, M. (2010). Tortuosity model for fixed beds randomly packed with identical particles. Chemical Engineering Science, 65(5):1891-1896.

Latief, F., Biswal, B., Fauzi, U., and Hilfer, R. (2010). Continuum reconstruction of the pore scale microstructure for fontainebleau sandstone. Physica A: Statistical Mechanics and its Applications, 389(8):1607-1618.

Lee, T.-C., Kashyap, R. L., and Chu, C.-N. (1994). Building skeleton models via 3-d medial surface axis thinning algorithms. CVGIP: Graphical Models and Image Processing, 56(6):462-478.

Lemaitre, R. and Adler, P. M. (1990). Fractal porous media iv: three-dimensional stokes flow through random media and regular fractals. Transport in Porous Media, 5(4):325-340.

Li, W., Zou, C.-c., Wang, H., and Peng, C. (2017). A model for calculating the formation resistivity factor in low and middle porosity sandstone formations considering the effect of pore geometry. Journal of Petroleum Science and Engineering, 152:193-203.

Lindquist, W. B., Lee, S.-M., Coker, D. A., Jones, K. W., and Spanne, P. (1996). Medial axis analysis of void structure in three-dimensional tomographic images of porous media. Journal of Geophysical Research: Solid Earth, 101(B4):8297-8310.

Litster, S., Epting, W., Wargo, E., Kalidindi, S., and Kumbur, E. (2013). Morphological analyses of polymer electrolyte fuel cell electrodes with nano-scale computed tomography imaging. Fuel Cells, 13(5):935-945.

Lu, X. (1997). Application of the nernst-einstein equation to concrete. Cement and Concrete Research, $27(2): 293-302$. 
Luo, L., Yu, B., Cai, J., and Zeng, X. (2014). Numerical simulation of tortuosity for fluid flow in two-dimensional pore fractal models of porous media. Fractals, 22(04):1450015.

Malek, K. and Coppens, M.-O. (2003). Knudsen self-and fickian diffusion in rough nanoporous media. The Journal of Chemical Physics, 119(5):2801-2811.

Masís-Meléndez, F., Deepagoda, T. C., de Jonge, L. W., Tuller, M., and Moldrup, P. (2014). Gas diffusion-derived tortuosity governs saturated hydraulic conductivity in sandy soils. Journal of Hydrology, 512:388-396.

Matyka, M., Khalili, A., and Koza, Z. (2008). Tortuosity-porosity relation in porous media flow. Physical Review E, 78(2):026306.

Matyka, M. and Koza, Z. (2012). How to calculate tortuosity easily? In AIP Conference Proceedings 4 , volume 1453, pages 17-22. AIP.

Mauret, E. and Renaud, M. (1997). Transport phenomena in multi-particle systems-i. limits of applicability of capillary model in high voidage beds-application to fixed beds of fibers and fluidized beds of spheres. Chemical Engineering Science, 52(11):1807-1817.

Moctezuma-Berthier, A., Vizika, O., and Adler, P. M. (2002). Macroscopic conductivity of vugular porous media. Transport in Porous Media, 49(3):313-332.

Moctezuma-Berthier, A., Vizika, O., Thovert, J., and Adler, P. M. (2004). One-and two-phase permeabilities of vugular porous media. Transport in Porous Media, 56(2):225-244.

Moldrup, P., Olesen, T., Komatsu, T., Schjønning, P., and Rolston, D. (2001). Tortuosity, diffusivity, and permeability in the soil liquid and gaseous phases. Soil Science Society of America Journal, 65(3):613-623.

Montes, J., Cuevas, F., and Cintas, J. (2007). Electrical and thermal tortuosity in powder compacts. Granular Matter, 9(6):401-406.

Moon, C. and Andrew, M. (2019). Intergranular pore structures in sandstones. http://www. digitalrocksportal.org/projects/222.

Mota, M., Teixeira, J. A., Bowen, W. R., and Yelshin, A. (2001). Binary spherical particle mixed beds: porosity and permeability relationship measurement.

Moukalled, F., Mangani, L., Darwish, M., et al. (2016). The finite volume method in computational

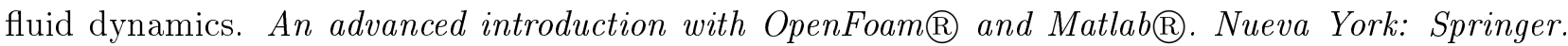
Recuperado de http://www. gidropraktikum. narod. ru/Moukalled-et-al-FVM-OpenFOAM-Matlab. pdf.

Mualem, Y. and Friedman, S. (1991). Theoretical prediction of electrical conductivity in saturated and unsaturated soil. Water Resources Research, 27(10):2771-2777.

Münch, B. and Holzer, L. (2008). Contradicting geometrical concepts in pore size analysis attained with electron microscopy and mercury intrusion. Journal of the American Ceramic Society, 91(12):40594067.

Munroe, P. R. (2009). The application of focused ion beam microscopy in the material sciences. Materials Characterization, 60(1):2-13.

Nakashima, Y. and Kamiya, S. (2007). Mathematica programs for the analysis of three-dimensional pore connectivity and anisotropic tortuosity of porous rocks using x-ray computed tomography image data. Journal of Nuclear Science and Technology, 44(9):1233-1247. 
Nakashima, Y. and Watanabe, Y. (2002). Estimate of transport properties of porous media by microfocus X-ray computed tomography and random walk simulation. Water Resources Research, 38(12).

Ning, Y., He, S., Liu, H., Wang, H., Qin, G., et al. (2016). A rigorous upscaling procedure to predict macro-scale transport properties of natural gas in shales by coupling molecular dynamics with lattice boltzmann method. In SPE Annual Technical Conference and Exhibition. Society of Petroleum Engineers.

Olives, R. and Mauran, S. (2001). A highly conductive porous medium for solid-gas reactions: effect of the dispersed phase on the thermal tortuosity. Transport in Porous Media, 43(2):377-394.

OpenLB (2019). Website. https://www.openlb.net/.

Ostadi, H., Rama, P., Liu, Y., Chen, R., Zhang, X., and Jiang, K. (2010). 3d reconstruction of a gas diffusion layer and a microporous layer. Journal of Membrane Science, 351(1-2):69-74.

Otsu, N. (1979). A threshold selection method from gray-level histograms. IEEE Transactions on Systems, Man, and Cybernetics, 9(1):62-66.

Palabos (2019). Univeristy of Geneva. Geneva, Switzerland. Website. https://palabos .unige.ch/.

Pardo-Alonso, S., Vicente, J., Solórzano, E., Rodriguez-Perez, M. Á., and Lehmhus, D. (2014). Geometrical tortuosity 3d calculations in infiltrated aluminium cellular materials. Procedia Materials Science, $4: 145-150$.

Patel, K. K., Paulsen, J. M., and Desilvestro, J. (2003). Numerical simulation of porous networks in relation to battery electrodes and separators. Journal of Power Sources, 122(2):144-152.

Pazdniakou, A. and Adler, P. M. (2012). Lattice spring models. Transport in Porous Media, 93(2):243262.

Peng, S., Marone, F., and Dultz, S. (2014). Resolution effect in x-ray microcomputed tomography imaging and small pore's contribution to permeability for a berea sandstone. Journal of Hydrology, 510:403-411.

Petersen, L. W., Moldrup, P., Jacobsen, O. H., and Rolston, D. (1996). Relations between specific surface area and soil physical and chemical properties. Soil Science, 161(1):9-21.

Piller, M., Schena, G., Nolich, M., Favretto, S., Radaelli, F., and Rossi, E. (2009). Analysis of hydraulic permeability in porous media: from high resolution x-ray tomography to direct numerical simulation. Transport in Porous Media, 80(1):57.

Pisani, L. (2011). Simple expression for the tortuosity of porous media. Transport in Porous Media, $88(2): 193-203$.

Plawsky, J. L. (2020). Transport phenomena fundamentals. CRC press.

Prachayawarakorn, S. and Mann, R. (2007). Effects of pore assembly architecture on catalyst particle tortuosity and reaction effectiveness. Catalysis Today, 128(1-2):88-99.

Pudney, C. (1998). Distance-ordered homotopic thinning: a skeletonization algorithm for 3d digital images. Computer Vision and Image Understanding, 72(3):404-413.

Ringer, S. and Hono, K. (2000). Microstructural evolution and age hardening in aluminium alloys: atom probe field-ion microscopy and transmission electron microscopy studies. Materials Characterization, 44(1-2):101-131. 
Salles, J., Thovert, J., and Adler, P. M. (1993). Deposition in porous media and clogging. Chemical Engineering Science, 48(16):2839-2858.

San Wu, Y., van Vliet, L. J., Frijlink, H. W., and van der Voort Maarschalk, K. (2006). The determination of relative path length as a measure for tortuosity in compacts using image analysis. European Journal of Pharmaceutical Sciences, 28(5):433-440.

Saomoto, H. and Katagiri, J. (2015). Direct comparison of hydraulic tortuosity and electric tortuosity based on finite element analysis. Theoretical and Applied Mechanics Letters, 5(5):177-180.

Schlüter, S., Sheppard, A., Brown, K., and Wildenschild, D. (2014). Image processing of multiphase images obtained via x-ray microtomography: a review. Water Resources Research, 50(4):3615-3639.

Schwartz, L. M., Garboczi, E. J., and Bentz, D. P. (1995). Interfacial transport in porous media: Application to dc electrical conductivity of mortars. Journal of Applied Physics, 78(10):5898-5908.

Sethian, J. A. (1996). A fast marching level set method for monotonically advancing fronts. Proceedings of the National Academy of Sciences, 93(4):1591-1595.

Shah, S., Gray, F., Crawshaw, J., and Boek, E. (2016). Micro-computed tomography pore-scale study of flow in porous media: Effect of voxel resolution. Advances in Water Resources, 95:276-287.

Shanti, N. O., Chan, V. W., Stock, S. R., De Carlo, F., Thornton, K., and Faber, K. T. (2014). X-ray micro-computed tomography and tortuosity calculations of percolating pore networks. Acta Materialia, 71:126-135.

Shearing, P. R., Brandon, N., Gelb, J., Bradley, R., Withers, P., Marquis, A., Cooper, S., and Harris, S. (2012). Multi length scale microstructural investigations of a commercially available li-ion battery electrode. Journal of The Electrochemical Society, 159(7):A1023-A1027.

Shen, L. and Chen, Z. (2007). Critical review of the impact of tortuosity on diffusion. Chemical Engineering Science, 62(14):3748-3755.

Sheppard, A. and Prodanovic, M. (2015). Network generation comparison forum. http://www . digitalrocksportal.org/projects/16.

Simpleware (2019). Synopsys. California, USA. Website. http://www. simpleware.com.

Sing, K. S. and Williams, R. T. (2004). The use of molecular probes for the characterization of nanoporous adsorbents. Particle \& Particle Systems Characterization: Measurement and Description of Particle Properties and Behavior in Powders and Other Disperse Systems, 21(2):71-79.

Smith, J., Chen, A., Gostovic, D., Hickey, D., Kundinger, D., Duncan, K., DeHoff, R., Jones, K., and Wachsman, E. (2009). Evaluation of the relationship between cathode microstructure and electrochemical behavior for sofcs. Solid State Ionics, 180(1):90-98.

Sobieski, W., Matyka, M., Gołembiewski, J., and Lipiński, S. (2018). The path tracking method as an alternative for tortuosity determination in granular beds. Granular Matter, 20(4):72.

Spanne, P., Thovert, J. F., Jacquin, C. J., Lindquist, W. B., Jones, K. W., and Adler, P. M. (1994). Synchrotron computed microtomography of porous media: topology and transports. Physical Review Letters, 73(14):2001.

Srisutthiyakorn, N. and Mavko, G. M. (2017). What is the role of tortuosity in the kozeny-carman equation? Interpretation, 5(1):SB57-SB67.

Stenzel, O., Pecho, O., Holzer, L., Neumann, M., and Schmidt, V. (2016). Predicting effective conductivities based on geometric microstructure characteristics. AIChE Journal, 62(5):1834-1843. 
Stutzman, P. (2004). Scanning electron microscopy imaging of hydraulic cement microstructure. Cement and Concrete Composites, 26(8):957-966.

Sun, F., Zielke, L., Marköter, H., Hilger, A., Zhou, D., Moroni, R., Zengerle, R., Thiele, S., Banhart, J., and Manke, I. (2016). Morphological evolution of electrochemically plated/stripped lithium microstructures investigated by synchrotron x-ray phase contrast tomography. ACS Nano, 10(8):79907997.

Sun, W. and Wong, T.-f. (2018). Prediction of permeability and formation factor of sandstone with hybrid lattice boltzmann/finite element simulation on microtomographic images. International Journal of Rock Mechanics and Mining Sciences, 106:269-277.

Sun, W. C., Andrade, J. E., and Rudnicki, J. W. (2011). Multiscale method for characterization of porous microstructures and their impact on macroscopic effective permeability. International Journal for Numerical Methods in Engineering, 88(12):1260-1279.

Taiwo, O. O., Finegan, D. P., Eastwood, D. S., Fife, J. L., Brown, L. D., Darr, J. A., Lee, P. D., Brett, D. J., and Shearing, P. R. (2016). Comparison of three-dimensional analysis and stereological techniques for quantifying lithium-ion battery electrode microstructures. Journal of Microscopy, $263(3): 280-292$.

Tariq, F., Yufit, V., Kishimoto, M., Shearing, P., Menkin, S., Golodnitsky, D., Gelb, J., Peled, E., and Brandon, N. (2014). Three-dimensional high resolution x-ray imaging and quantification of lithium ion battery mesocarbon microbead anodes. Journal of Power Sources, 248:1014-1020.

Thovert, J., Wary, F., and Adler, P. M. (1990). Thermal conductivity of random media and regular fractals. Journal of Applied Physics, 68(8):3872-3883.

Thovert, J. F., Salles, J., and Adler, P. M. (1993). Computerized characterization of the geometry of real porous media: their discretization, analysis and interpretation. Journal of microscopy, 170(1):65-79.

Tjaden, B., Brett, D. J., and Shearing, P. R. (2018). Tortuosity in electrochemical devices: a review of calculation approaches. International Materials Reviews, 63(2):47-67.

Tjaden, B., Finegan, D. P., Lane, J., Brett, D. J., and Shearing, P. R. (2017). Contradictory concepts in tortuosity determination in porous media in electrochemical devices. Chemical Engineering Science, 166:235-245.

Tjaden, B., Lane, J., Withers, P. J., Bradley, R. S., Brett, D. J., and Shearing, P. R. (2016). The application of $3 \mathrm{~d}$ imaging techniques, simulation and diffusion experiments to explore transport properties in porous oxygen transport membrane support materials. Solid State Ionics, 288:315-321.

Torquato, S. (2002). Statistical description of microstructures. Annual Review of Materials Research, $32(1): 77-111$.

Tranter, T., Kok, M., Lam, M., and Gostick, J. (2019). pytrax: A simple and efficient random walk implementation for calculating the directional tortuosity of images. SoftwareX, 10:100277.

Tye, F. (1983). Tortuosity. Journal of Power Sources, 9:89-100.

Van Brakel, J. and Heertjes, P. (1974). Analysis of diffusion in macroporous media in terms of a porosity, a tortuosity and a constrictivity factor. International Journal of Heat and Mass Transfer, 17(9):1093-1103.

Vervoort, R. and Cattle, S. (2003). Linking hydraulic conductivity and tortuosity parameters to pore space geometry and pore-size distribution. Journal of Hydrology, 272(1-4):36-49. 
Vogel, H.-J., Weller, U., and Schlüter, S. (2010). Quantification of soil structure based on minkowski functions. Computers \& Geosciences, 36(10):1236-1245.

Wargo, E., Kotaka, T., Tabuchi, Y., and Kumbur, E. (2013). Comparison of focused ion beam versus nano-scale x-ray computed tomography for resolving 3-d microstructures of porous fuel cell materials. Journal of Power Sources, 241:608-618.

Weerts, A., Bouten, W., and Verstraten, J. (1999). Simultaneous measurement of water retention and electrical conductivity in soils: Testing the mualem-friedman tortuosity model. Water Resources Research, 35(6):1781-1787.

Weerts, A., Kandhai, D., Bouten, W., and Sloot, P. (2001). Tortuosity of an unsaturated sandy soil estimated using gas diffusion and bulk soil electrical conductivity. Soil Science Society of America Journal, 65(6):1577-1584.

Wiegmann, A. and Zemitis, A. (2006). Ej-heat: A fast explicit jump harmonic averaging solver for the effective heat conductivity of composite materials.

Wildenschild, D. and Sheppard, A. P. (2013). X-ray imaging and analysis techniques for quantifying pore-scale structure and processes in subsurface porous medium systems. Advances in Water Resources, 51:217-246.

Wilson, J. R., Kobsiriphat, W., Mendoza, R., Chen, H.-Y., Hiller, J. M., Miller, D. J., Thornton, K., Voorhees, P. W., Adler, S. B., and Barnett, S. A. (2006). Three-dimensional reconstruction of a solid-oxide fuel-cell anode. Nature Materials, 5(7):541.

Winsauer, W. O., Shearin Jr, H., Masson, P., and Williams, M. (1952). Resistivity of brine-saturated sands in relation to pore geometry. AAPG Bulletin, 36(2):253-277.

Wu, Y. S., Frijlink, H., et al. (2006). The determination of relative path length as a measure for tortuosity in compacts using image analysis. European Journal of Pharmaceutical sciences: official journal of the European Federation for Pharmaceutical Sciences, 28(5):433-440.

Wyllie, M., Rose, W. D., et al. (1950). Some theoretical considerations related to the quantitative evaluation of the physical characteristics of reservoir rock from electrical log data. Journal of Petroleum Technology, 2(04):105-118.

Wyllie, M. and Spangler, M. (1952). Application of electrical resistivity measurements to problem of fluid flow in porous media. AAPG Bulletin, 36(2):359-403.

Yang, K., Li, M., Ling, N. N., May, E. F., Connolly, P. R., Esteban, L., Clennell, M. B., Mahmoud, M., El-Husseiny, A., Adebayo, A. R., et al. (2019). Quantitative tortuosity measurements of carbonate rocks using pulsed field gradient nmr. Transport in Porous Media, pages 1-19.

Zacharias, N. A., Nevers, D. R., Skelton, C., Knackstedt, K., Stephenson, D. E., and Wheeler, D. R. (2013). Direct measurements of effective ionic transport in porous li-ion electrodes. Journal of The Electrochemical Society, 160(2):A306-A311.

Zhang, X. and Knackstedt, M. A. (1995). Direct simulation of electrical and hydraulic tortuosity in porous solids. Geophysical Research Letters, 22(17):2333-2336.

Zhao, Y., Liu, S., Elsworth, D., Jiang, Y., and Zhu, J. (2014). Pore structure characterization of coal by synchrotron small-angle x-ray scattering and transmission electron microscopy. Energy \& Fuels, 28(6):3704-3711. 
Zielinski, T. (2012). Inverse identification and microscopic estimation of parameters for models of sound absorption in porous ceramics. In Proceedings of International Conference on Noise and Vibration Engineering (ISMA2012)/International Conference on Uncertainty in Structural Dynamics (USD2012), pages $95-108$. 